Скопје, Македонија

\title{
SKAND THEORY AND ITS APPLICATIONS. (A NEW LOOK AT NON-WELL-FOUNDED SETS)
}

\author{
JU. T. LISICA
}

\begin{abstract}
A new mathematical object called a skand is introduced, which turns out in general to be a non-well-founded set. Skands of finite lengths are ordinary well-founded sets, and skands of very long length (like the hyper-skand of all ordinals) are hyper-classes.

Self-similar skands are also considered, and they clarify the reflexivity of sets, i.e., the meaning of the relation $X \in X$; in particular, self-similar skands considered as non-well-founded sets are always reflexive, but not vice versa. The existence of self-similar skands shows at once that all the well-known set-theoretical paradoxes are not paradoxes at all, and hence are not necessarily fatal for any set theory. E.g., the inconsistency of Russell's "set" $R=\{X \mid X \notin X\}$ is proved here not with the help of Russell's paradox (as it is traditionally given, which is incorrect), but via a simple method of the maximality (universality) of $R$ which goes back to Cantor and is also applied to other set-theoretical paradoxes.

Generalized skands are also defined and a new look at the generalized skand-class of all ordinals is demonstrated. In particular, the last (class) ordinal called the eschaton is defined.

The next application of skand theory is a description of all epsilonnumbers in the sense of Cantor. Another application is a generalized theory of one-dimensional continua of arbitrary powers and the construction of generalized real numbers as a non-Archimedean straight line of arbitrary power, and the introduction of the absolute continuum and the absolute straight line as the hyper-classes nearest to the class of sets.
\end{abstract}

2010 Mathematics Subject Classification. Primary 03E65, 03C62, 54 G99.

Key words and phrases. Skand, Russell's paradox, non-well-founded sets, reflexive sets, eschaton, epsilon-numbers, generalized rationals and reals, straight line of a large power, generalized continuum. 


\section{Epigraphs}

A parson had a hound-dog,
One he loved a lot.
It ate a piece of mutton,
For which he had it shot.
He buried the hound,
Then wrote on its mound,
That
A parson had a hound-dog, etc.
(ad infinitum and ad imum).
[Free translation of a Russian children's
ditty.]

Once a four-year-old son returned home from kindergarten, where he had been told that his father was a mathemati-

cian.

being".

When the son saw his father again, he asked him:

"Is it true, Daddy, that you are a mathematician?"

"Yes, sonny, it is," was the answer.

"Well," responded the son, "can you count to the last number?"

"Ummm...ummmm," mumbled the father, stumped.

[Dialogue with a child which is in fact a problem of mathematical eschatology:

"What is the 'É $\sigma \chi \alpha \tau o \nu$ or ad imum?"]

"A soul is only a skand, i.e., an accidental aggregation of

[From Buddhist doctrine.]

"The content of a concept diminishes as its extension increases; if its extension becomes all-embracing, its content must vanish altogether".

[Gottlob Frege, "The Foundations of Arithmetic".]

\section{Introduction}

We are going to clarify the notion of reflexivity in Set Theory, i.e., the meaning of a binary relation $X \in Y$ in the case when $Y=X$, that is $X \in X$. As to Russell himself, the relation $X \in X$ "must be always meaningless" [58], p. 81, since he was seriously frightened by this paradox, which was later on named after him: "Thus $X \in X$ was held to be meaningless, 
because $\in$ requires that the relatum should be a class composed of objects which are of the type of the referent" ([54], Chap. X, p. 107). Moreover, he went further and concluded that $X \notin X$ "must be always meaningless", too. ([58], p. 81). He wrote: "If $\alpha$ is a class, the statement ' $\alpha$ is not a member of $\alpha$ ' is always meaningless, and there is therefore no sense in the phrase "the class of those classes which are not members of themselves'" ([58], p. 66). In particular, Russell wrote: "A class consisting of only one member must not be identical with that one member". And he added immediately " $X=\{X\}$ must be absolutely meaningless, not simply false" ([58], p. 81). In this paper we shall see when Russell was right and when was he not.

In particular, we shall show that in many well-known formal systems (set theories) Russell's paradox is not a paradox at all, and hence the proof of the Proposition that Russell's collection $R=\{X \mid X \notin X\}$ is a proper class, not a set, via Russell's paradox, is not correct (it was a logical mistake in the propositional calculus, at least within set theories with the axiom of regularity). We prove this Proposition by the following

Maximality Principle. If there exists a maximal (universal) collection $X$ (sets, classes, hyper-classes), given by some property, predicate, etc., then any assertion which implies the existence of a new element $x$ with the same property and $x \notin X$ is false.

2. Premises, notations, some history, and purpose of paper

At the beginning we start out within a von Neumann-Bernays-Gödeltype set theory ( $N B G$ for short), i.e., the theory of first-order logic with equality (i.e., with respect to the given definition of equality $A_{1}^{2}(X, Y)$ ) with a syntactic or proof-theoretic side and a semantic or model-theoretic side (see [10], p. 7) with only the predicate letter $A_{2}^{2}(X, Y)$, which is the binary relation $X \in Y$, for short. The proper axioms of $N B G$ consist of general axioms, class-formation axioms and set-formation axioms together with the axiom of choice AC and the axiom of foundation FA (see, e.g., in [41], p. 225-286; we have changed only notation: $N B G+(\mathbf{A C})+(\boldsymbol{R e g})$ on $N B G)$. The basic set theory $N B G$ can be considered with individuals (e.g., [41], p. 297-304) called sometimes atoms or urelements, i.e., mathematical objects which are neither sets nor classes and which have no members, or $N B G$ can be considered without them; it does not matter. In the latter case the only individual is just the empty set \{\}$=\emptyset$ and Set Theory in this case is often called the theory of "pure" sets.

To be more precise and definite we denote by $N B G[\mathcal{U}]$ the set theory with of individuals (atoms, urelements), the class of all sets of $N B G[\mathcal{U}]$ by $\mathbf{V}[\mathcal{U}]$, and the set or class of individuals (atoms, urelements) by $\mathcal{U} ; \mathbf{V}$ is 
the class of all sets in the theory $N B G$ of "pure" sets, i.e., when $\mathcal{U}=\emptyset$. In $N B G[\mathcal{U}]$ because of $\mathbf{F A}$ the class $\mathbf{V}[\mathcal{U}]$ of all sets turns out to be the class $\mathbf{W F}$ of all well-founded sets. The universal class of elements $\mathbf{U}[\mathcal{U}]$ is the union $\mathbf{V}[\mathcal{U}] \cup \mathcal{U}$ of $\mathbf{V}[\mathcal{U}]$ and $\mathcal{U}$. Note that $\mathbf{V}[\mathcal{U}] \cap \mathcal{U}=\emptyset$.

We also denote the class of all ordinals by On and the class of all cardinals by Card.

Then we shall consider $N B G[\mathcal{U}]^{-}$, i.e., $N B G[\mathcal{U}]$ without the axiom of foundation $\mathbf{F A}$, and instead of the axiom of choice we use the axiom $\mathbf{N}$ of von Neumann, $\mathbf{V}[\mathcal{U}]^{-} \approx \mathbf{O}$; i.e., these classes are bijective, which in the absence of foundation is stronger than choice.

There is an important distinction in $N B G[\mathcal{U}]$ as well as in $N B G[\mathcal{U}]^{-}$Set Theories between three kinds of objects: individuals, sets (usually called "small" classes), and classes (usually called "large" classes or more often proper classes). Individuals do not contain any elements but can be elements of non-empty sets and classes; non-empty sets can contain individuals and sets as elements but do not contain classes as elements, and sets can be elements of non-empty sets and clases; classes can obtain individuals and sets as elements but can not be elements of individuals, sets or classes. The only indivinual $\emptyset$ is called a set; others are not sets or classes.

There are two ways to distinguish sets and classes (i.e., proper classes) in addition to axioms for sets. The first of them is the following: a subclass $X \subset \mathbf{U}[\mathcal{U}]$ of $\mathbf{U}[\mathcal{U}]$ is a set if and only if there exists a one-element object (singleton) $\{X\} \in \mathbf{V}[\mathcal{U}]$; the second one: a subclass $X \subset \mathbf{U}[\mathcal{U}]$ of $\mathbf{U}[\mathcal{U}]$ is a set if and only if there is no bijection $X$ on $\mathbf{U}[\mathcal{U}]$. Otherwise, $X \subseteq \mathbf{U}[\mathcal{U}]$ is not a set but a subclass (i.e., proper subclass) of $\mathbf{U}[\mathcal{U}]$ (further, in short, a class). Moreover, all subclasses (which are not sets) of $\mathbf{U}[\mathcal{U}]$ are bijective to each other. Notice also that when we deal with classes (i.e., proper classes) we speak in the language of their elements but not of them as wholes or as units. In other words, sets are arguments (elements of $\mathbf{V}[\mathcal{U}]$ ), and classes are extensions of some predicates. In formal systems one uses the following notations: $C l s(X)$ as " $X$ is a class", $M(X)$ as " $X$ is a set", i.e., $(\exists Y)(X \in Y), \operatorname{Pr}(X)$ as " $X$ is a proper class, i.e., $C l s(X) \wedge \neg M(X)$, $U r(X)$ as " $X$ is an urelement" and $E l(X)$ as " $X$ is an elements" i.e., $E l(X)=M(X) \vee U r(X)$. (See [41], Chap. 4, p. 297.) For simplicity we avoid here almost these notations.

Proposition 1. If theory $N B G^{-}$is consistent, then $N B G$ is also consistent.

Proof see in [40] or in [41], Chap. 4, 4. 86. 
Moreover, a model of $N B G$ is built by the following transfinite recursion:

$$
\begin{gathered}
\Psi(0)=\emptyset \\
\Psi\left(\alpha^{\prime}\right)=\mathcal{P} \Psi(\alpha) \\
\lim (\lambda) \Longrightarrow \Psi(\lambda)=\bigcup_{\beta<\lambda} \Psi(\beta) \\
\mathbf{H}^{\prime}=\bigcup_{\alpha \in \mathbf{O} \mathbf{n}} \Psi(\alpha) .
\end{gathered}
$$

Here and below $\alpha^{\prime}=\alpha+1, \alpha \in \mathbf{O n}$, and $\mathcal{P}$ - the functor "set of all subsets".

Moreover, $\mathbf{H}^{\prime}$ determies an inner model of $N B G^{-}$and the axiom of foundation $\mathbf{F A}$ is equivalent to the statement that $\mathbf{V}=\mathbf{H}^{\prime}$ (Ibidem).

Proposition 2. Theory $N B G[\mathcal{U}]$ is consistent if and only if $N B G$ is consistent.

Proof see in [47] or in [41], Chap. 4, Proposition 4.50, p. 301-302.

A model of $N B G[\mathcal{U}]$ is built by the following transfinite recursion.

If $\mathcal{U}$ is a set, then

$$
\begin{gathered}
\Xi(0)=\mathcal{U} \\
\Xi\left(\alpha^{\prime}\right)=\mathcal{P} \Xi(\alpha) \\
\lim (\lambda) \Longrightarrow \Xi(\lambda)=\bigcup_{\beta<\lambda} \Xi(\beta) \\
\mathbf{H}^{\prime}[\mathcal{U}]=\bigcup_{\alpha \in \mathbf{O} \mathbf{n}} \Xi(\alpha) .
\end{gathered}
$$

Moreover, $\mathbf{H}^{\prime}[\mathcal{U}]$ determines an inner model of $N B G[\mathcal{U}]^{-}$and the axiom of foundation $\mathbf{F A}$ is equivalent to the statement that $\mathbf{V}[\mathcal{U}]=\mathbf{H}^{\prime}[\mathcal{U}]$.(Ibidem.)

If $\mathcal{U}$ is a class, then for each subset $L \subset \mathcal{U}$ and any ordinal $\gamma \in \mathbf{O n}$ we define a set $\Xi_{L}^{\gamma}$ be the following transfinite recursion:

$$
\begin{gathered}
\Xi_{L}^{\gamma}(0)=L \\
\Xi_{L}^{\gamma}\left(\alpha^{\prime}\right)=\Xi_{\mathcal{P}}^{\gamma}(\alpha), \quad \alpha^{\prime}<\gamma, \\
\lim (\lambda) \Longrightarrow \Xi_{L}^{\gamma}(\lambda)=\bigcup_{\beta<\lambda} \Xi_{L}^{\gamma}(\beta), \quad \lambda<\gamma .
\end{gathered}
$$

Let $\mathbf{H}[\mathcal{U}]$ be the class of all elements $M$ such that for some set $L$ and ordinal $\gamma, M$ is in the range of $\Xi_{L}^{\gamma}$. Then $\mathbf{H}[\mathcal{U}]$ determines a model of $N B G[\mathcal{U}]$ and the foundation axiom FA holds if and only if $\mathbf{H}[\mathcal{U}]=\mathbf{U}[\mathcal{U}]$. Moreover, $\mathbf{H}[\mathcal{U}]$ determines an inner model of $N B G[\mathcal{U}]^{-}$. (Ibidem.)

Recall that a set $X$ is well-founded (or ordinary) if there is no infinite $\in$-descending chains, in other words, if every $\in$-descending chain in $X$ is finite, i.e., for each $x_{0} \in X$, every $\in$-descending chain starting with $x_{0}$ can be at most the following one: $x_{0} \ni x_{1} \ni x_{2} \ni \ldots \ni x_{n}$, where $x_{n}$ is some individual or the empty set; otherwise it is non-well-founded or "extraordinary", i.e., there exist infinite $\in$-descending chains $x_{0} \ni x_{1} \ni$ 
$x_{2} \ni \ldots \ni x_{n} \ni \ldots$ The distinction between well-founded and non-wellfounded sets was first articulated by Mirimanoff [43] and in his terminology the distinction was between "ordinary" and "extraordinary" sets. Later on, von Neumann [50] proposed an axiom of regularity ("Restrictive Axiom" in [5] and "Axiom der Fundierung" in [60], i.e. the Foundation Axiom FA) which excluded Mirimanoff's extraordinary sets, because according to the axiom any "descending" sequence terminates, i.e., reaches its bottom or "foundation". More precisely, the Axiom of Regularity in $N B G[\mathcal{U}]$ is the following:

$$
(\forall X)(X \neq \emptyset) \Longrightarrow(\exists u)(u \in X \wedge \neg(\exists v)(v \in X \wedge v \in u)))
$$

and together with the Axiom of Choice it is equivalent to the Axiom of Foundation (see [41], Chap. 4, Proposition 4.44).

The restriction axiom as FA was very important, first of all, for completeness of the extensionality axiom Ext, because in $N B G[\mathcal{U}]^{-}$it is impossible to prove in general that for different sets $X$ and $Y$ the two-element set $Z=\{X, Y\}$, which always exists by the axiom of pairing, $Z=X$, or $Z=Y$, or $Z$ is different from $X$ and $Y$. Only by means of FA can one prove that $\{X, Y\}$ is different from $X$ and $Y$. Secondly, FA avoids vicious circle phenomena, i.e., there is no set $X$ such that $X \ni X_{1} \ni \ldots \ni X_{n} \ni X$; in particular, the reflexive sets $X \in X$, which appeared to be a source of paradoxes (which was actually not true), e.g., Russell, with reference to $\mathrm{H}$. Poincaré wrote: "An analysis of the paradoxes to be avoided shows that they all result from a certain kind of vicious circle" [58], p. 39. And Russell had formulated his famous "vicious-circle principle" as follows: "Whatever involves all of a collection must not be one of the collection" [58], p. 40. Thus, with the help of the Foundation Axiom, Set Theory has been succesfully developed and the "vicious-circle principle" has been satisfied.

Nevertheless, many real problems concern circular phenomena in logic (first of all, Russell's paradox itself, because it concerns the relation $R \in R$, and therefore the non-well-founded set $R$; and also, e.g., the treatment of Liar-like paradoxes, etc.); linguistics, computer science, graph theory, game theory, streams, etc., all need circular models which lie beyond the universe of well-founded sets. At last, there is a lack of investigation of reflexive sets and Mirimanoff extraordinary sets which are in general many-valued. Even a real example of a set $X$ which is an element of itself is absent in almost monographs on Set Theory except those like "set of all sets", e.g., [27], p. 5, or in Russell's paradox $R \in R$ in proving that $R$ is not a set; these examples are fakes. Only later in monographs on "non-well-founded sets" real examples appeared. 
From the early 20th century, many authors actually proposed their own anti-foundation axioms (AFA for short) enriching and extending the WellFounded Universe by the AFA-Universe, e.g., [25], [7], [50], [55], [26] and others. All of them proposed theories of possibly non-well-founded sets which are consistent, assuming that $N B G^{-}$is consistent. Nevertheless, the four axiom systems mentioned are non-comparable, and each one differs from the others in the strengthening of the extensionality criterion for set equality.

In the present paper we introduce a new object called a skand (Sanskrit: jump, skip). Skands are "definite and separate" objects ("bestimmten wohlunterschiedenen Objekten" according to Cantor [15], p. 481) and can be considered as elements of the class $\mathbf{V}[\mathcal{U}]^{-}$of all sets in $N B G^{-}$, and they also enrich $\mathbf{V}[\mathcal{U}]$; i.e., they can be well-founded or non-well-founded sets. Moreover, skands are essential extensions of some (not all) of Mirimanoff's extraordinary sets.

The class of all skands generates a class (i.e., proper class) $\mathbf{V}[\mathcal{U}]^{(1)}$ which is a subclass of the class $\mathbf{V}[\mathcal{U}]^{-}$by forming objects $X$ whose elements are ordinary sets or skands such that $\{X\}$ exists. Clearly, $\mathbf{V}[\mathcal{U}] \stackrel{\text { def }}{=} \mathbf{V}[\mathcal{U}]^{(0)} \subset$ $\mathbf{V}[\mathcal{U}]^{(1)} \subset \mathbf{V}[\mathcal{U}]^{-}$. Moreover, we successively continue such a process of enrichment for each ordinal number $\alpha$ and obtain the following embeddings: $\mathbf{V}[\mathcal{U}]^{(0)} \subset \mathbf{V}[\mathcal{U}]^{(1)} \subset \ldots \subset \mathbf{V}[\mathcal{U}]^{(\nu)} \subset \ldots \subset \mathbf{V}[\mathcal{U}]^{\Omega} \subset \mathbf{V}[\mathcal{U}]^{-}$, where $\mathbf{V}[\mathcal{U}]^{\Omega}=\bigcup_{\nu \in \mathbf{O} \mathbf{n}} \mathbf{V}[\mathcal{U}]^{(\alpha)}$. We do not say that this cumulative hierarchy exhausts $\mathbf{V}[\mathcal{U}]^{-}$, i.e., $\mathbf{V}[\mathcal{U}]^{\Omega}=\mathbf{V}[\mathcal{U}]^{-}$but it makes $\mathbf{V}[\mathcal{U}]^{-}$more structural.

\section{The notion of a skand}

Let $\left(\alpha_{0}, \alpha\right) \stackrel{\text { def }}{=}\left\{\alpha^{\prime} \in \mathbf{O n} \mid \alpha_{0} \leq \alpha^{\prime}<\alpha\right\}$ be a segment of On; if $\alpha_{0}=0$ and $\alpha \geq 1$, then we call $(0, \alpha)$ an initial segment of $\mathbf{O n}$.

Definition 1. Consider a system of embedded curly braces or wellordered set of embedding pairs of curly braces $\left\{\alpha_{0}\left\{\alpha_{0}+1 \cdots\left\{\alpha^{\prime} \cdots \alpha^{\prime}\right\} \cdots \alpha_{0}+1\right\} \alpha_{0}\right\}$, indexed by ordinals $\alpha^{\prime} \in\left(\alpha_{0}, \alpha\right)$. We call it a trivial skand of length $l=\alpha-\alpha_{0}$ and denote it by $\mathbf{e}_{\left(\alpha_{0}, \alpha\right)}$. It can be also called an empty skand because for each $\alpha^{\prime} \in\left(\alpha_{0}, \alpha\right)$ the set of all elements between two neighboring opening braces $\left\{\alpha^{\prime}\left\{\alpha^{\prime}+1\right.\right.$ or possibly between $\left\{\alpha^{\prime} \alpha^{\prime}\right\}$, if $\alpha^{\prime}=$ $\alpha-1$, is empty. In other words, each $\alpha^{\prime}$-component $\mathbf{e}_{\alpha^{\prime}}$ of $\mathbf{e}_{\left(\alpha_{0}, \alpha\right)}$ is empty. By a non-trivial skand $X_{\left(\alpha_{0}, \alpha\right)}$ of length $l=\alpha-\alpha_{0}$ we understand a non-trivial system of embedded curly braces, indexed by ordinals $\alpha^{\prime} \in\left(\alpha_{0}, \alpha\right)$; i.e., there is at least one index $\alpha^{\prime} \in\left(\alpha_{0}, \alpha\right)$, and elements $x_{0}, x_{1}, x_{2}, \ldots, x_{\lambda}, \ldots$ of $\mathbf{U}[\mathcal{U}]$ such that the $\alpha^{\prime}$-component $X_{\alpha^{\prime}} \stackrel{\text { def }}{=}$ 
$\left\{\alpha^{\prime} x_{0}, x_{1}, x_{2}, \ldots, x_{\lambda}, \ldots,\left\{\alpha^{\prime}+1\right.\right.$ (or $\left\{\alpha_{\alpha^{\prime}} x_{0}, x_{1}, x_{2}, \ldots, x_{\lambda}, \ldots \alpha^{\prime}\right\}$, if $\alpha^{\prime}=\alpha-1$ ) of $X_{\left(\alpha_{0}, \alpha\right)}$ is a set, i.e., $\left\{x_{0}, x_{1}, x_{2}, \ldots, x_{\lambda}, \ldots\right\} \in \mathbf{V}[\mathcal{U}]$ and denoted also as $X_{\alpha}$.

For simplicity we shall omit indexes $\alpha^{\prime}$ of braces or elements in the cases when it is clear what they are, e.g., for $\left\{\alpha_{\alpha^{\prime}} x_{0}^{\alpha^{\prime}}, x_{1}^{\alpha^{\prime}}, x_{2}^{\alpha^{\prime}}, \ldots, x_{\lambda_{\alpha^{\prime}}}^{\alpha^{\prime}}, \ldots,\left\{\alpha_{\alpha^{\prime}+1}\right.\right.$ or possibly $\left\{\alpha^{\prime} x_{0}^{\alpha^{\prime}}, x_{1}^{\alpha^{\prime}}, x_{2}^{\alpha^{\prime}}, \ldots, x_{\lambda_{\alpha^{\prime}}}^{\alpha^{\prime}}, \ldots \alpha^{\prime}\right\}$ in the case $\alpha^{\prime}=\alpha-1$, we write $\left\{x_{0}^{\alpha^{\prime}}, x_{1}^{\alpha^{\prime}}, x_{2}^{\alpha^{\prime}}, \ldots, x_{\lambda_{\alpha^{\prime}}}^{\alpha^{\prime}}, \ldots,\left\{\right.\right.$ and $\left\{x_{0}^{\alpha^{\prime}}, x_{1}^{\alpha^{\prime}}, x_{2}^{\alpha^{\prime}}, \ldots, x_{\lambda_{\alpha^{\prime}}}^{\alpha^{\prime}}, \ldots\right\}$ in the case $\alpha^{\prime}=$ $\alpha-1$, respectively; or even simplify to $\left\{x_{0}, x_{1}, x_{2}, \ldots, x_{\lambda}, \ldots,\{\right.$ and $\left\{x_{0}, x_{1}, x_{2}, \ldots, x_{\lambda}, \ldots\right\}$, respectively. All the more indexes $\alpha^{\prime}$ of braces are conditional, e.g., if $X_{(0, \alpha)}$ is a skand, then $Y_{(0, \alpha)}=\left\{X_{(0, \alpha)}\right\}$ whose components are $Y_{0}=\emptyset, Y_{1}=X_{0}, Y_{2}=X_{1}, \ldots, Y_{n+1}=X_{n}, \ldots$, and $Y_{\alpha^{\prime}}=X_{\alpha^{\prime}}$, for all $\omega \leq \alpha^{\prime}<\alpha$, is a skand, too.

For the purpose of interpreting skands as sets, we write a comma before the second open brace of non-trivial component $X_{\alpha^{\prime}}$ of $X_{\left(\alpha_{0}, \alpha\right)}$, i.e., $\left\{\alpha^{\prime} x_{0}, x_{1}, x_{2}, \ldots, x_{\lambda}, \ldots, \alpha^{\prime}+1\left\{\right.\right.$ or simply $\left\{x_{0}, x_{1}, x_{2}, \ldots, x_{\lambda}, \ldots,\{\right.$. It says that braces are not only syntactical but also semantic in the definition of skand.

Thus, a general form of an arbitrary skand is the following:

$$
X_{\left(\alpha_{0}, \alpha\right)}=\left\{x_{0}^{\alpha_{0}}, x_{1}^{\alpha_{0}}, \ldots, x_{\lambda_{\alpha_{0}}}^{\alpha_{0}}, \ldots,\left\{\ldots\left\{x_{0}^{\alpha^{\prime}}, x_{1}^{\alpha^{\prime}}, \ldots, x_{\lambda_{\alpha^{\prime}}}^{\alpha^{\prime}}, \ldots,\{\ldots\}\right\} \ldots\right\},\right.
$$

where components $X_{\alpha^{\prime}}=\left\{x_{0}^{\alpha^{\prime}}, x_{1}^{\alpha^{\prime}}, \ldots, x_{\lambda_{\alpha^{\prime}}}^{\alpha^{\prime}}, \ldots,\{\right.$ are sets, i.e.,

$$
\left\{x_{0}^{\alpha^{\prime}}, x_{1}^{\alpha^{\prime}}, \ldots, x_{\lambda_{\alpha^{\prime}}}^{\alpha^{\prime}}, \ldots\right\} \in \mathbf{V}[\mathcal{U}]
$$

or empty, i.e., $X_{\alpha^{\prime}}=\{\{$; if $\alpha$ is not a limit ordinal, then the last component $X_{\alpha-1}$ is an ordinary set, i.e., $\left\{x_{0}^{\alpha^{\prime}}, x_{1}^{\alpha^{\prime}}, \ldots, x_{\lambda_{\alpha^{\prime}}}^{\alpha^{\prime}}, \ldots\right\} \in \mathbf{V}[\mathcal{U}]$ or empty \{\} .

If all components $X_{\alpha^{\prime}}, \alpha_{0} \leq \alpha^{\prime}<\alpha$, of a skand $X_{\left(\alpha_{0}, \alpha\right)}$ are equal to the same set, e.g., $X=\left\{x_{0}, x_{1}, \ldots, x_{\lambda}, \ldots\right\}$, we shall denote this skand in a shorter way by $X_{\left(\alpha_{0}, \alpha\right)}(X)$; in particular, when $X=\{\gamma\}$, i.e., a one-element set $X$, we simplify the notation to $X_{\left(\alpha_{0}, \alpha\right)}(\gamma)$. If $\alpha_{0} \leq \alpha_{1}<\alpha_{2} \leq \alpha$, then together with $X_{\left(\alpha_{0}, \alpha\right)}$ we shall denote by $X_{\left(\alpha_{1}, \alpha_{2}\right)}$ the skand whose $\alpha^{\prime}$-components $X_{\alpha^{\prime}}, \alpha_{1} \leq \alpha^{\prime}<\alpha_{2}$, are the same as those of $X_{\left(\alpha_{0}, \alpha\right)}$, and $X_{\left(\alpha_{1}, \alpha_{2}\right)}$ is called a restriction of $X_{\left(\alpha_{0}, \alpha\right)}$ on $\left(\alpha_{1}, \alpha_{2}\right)$.

Definition 2. Two skands $X_{\left(\alpha_{0}, \alpha\right)}$ and $Y_{\left(\beta_{0}, \beta\right)}$ are called equal if the segments $\left(\alpha_{0}, \alpha\right)$ and $\left(\beta_{0}, \beta\right)$ are isomorphic as well-ordered sets, i.e., their "similarity" is given by $\varphi:\left(\alpha_{0}, \alpha\right) \rightarrow\left(\beta_{0}, \beta\right)$, and the corresponding $\alpha^{\prime}$ and $\beta^{\prime}$-components

$X_{\alpha^{\prime}}=\left\{\alpha^{\prime} x_{1}^{\alpha^{\prime}}, x_{2}^{\alpha^{\prime}}, \ldots, x_{\lambda}^{\alpha^{\prime}}, \ldots,\left\{\alpha^{\prime}+1\right.\right.$ and $Y_{\beta^{\prime}}=\left\{\beta_{\beta^{\prime}} y_{1}^{\beta^{\prime}}, y_{2}^{\beta^{\prime}}, \ldots, y_{\lambda}^{\beta^{\prime}}, \ldots,\left\{\beta_{\beta^{\prime}+1}\right.\right.$ as well as the last components $X_{\alpha^{\prime}}=\left\{\alpha^{\prime} x_{1}^{\alpha^{\prime}}, x_{2}^{\alpha^{\prime}}, \ldots, x_{\lambda}^{\alpha^{\prime}}, \ldots \alpha^{\prime}\right\}$ and $Y_{\beta^{\prime}}=\left\{\beta_{\beta^{\prime}} y_{1}^{\beta^{\prime}}, y_{2}^{\beta^{\prime}}, \ldots, y_{\lambda}^{\beta^{\prime}}, \ldots \beta^{\prime}\right\}$, if $\alpha^{\prime}=\alpha-1$ and $\beta^{\prime}=\beta-1$, are equal as sets in $N B G[\mathcal{U}]$; i.e., $\left\{x_{1}^{\alpha^{\prime}}, x_{2}^{\alpha^{\prime}}, \ldots, x_{\lambda}^{\alpha^{\prime}}, \ldots\right\}=\left\{y_{1}^{\beta^{\prime}}, y_{2}^{\beta^{\prime}}, \ldots, y_{\lambda}^{\beta^{\prime}}, \ldots\right\}, \alpha_{0} \leq \alpha^{\prime}<\alpha$ and $\beta_{0} \leq \beta^{\prime}<\beta$, respectively, where $\beta^{\prime}=\varphi\left(\alpha^{\prime}\right)$. 
It is clear that the relation of equality is an equivalent relation.

Remark 1. Actually, between each pair of braces $\{\{$ or \{\} of a skand $X_{\left(\alpha_{0}, \alpha\right)}$ there are different elements (sets or individuals), or their lack, which form a set; notice that the order in which the members of sets are written does not matter. In our notation we use $X_{\alpha^{\prime}}=\left\{x_{0}, x_{1}, \ldots, x_{\lambda}, \ldots,\left\{\right.\right.$ or $X_{\alpha^{\prime}}=$ $\left\{x_{0}, x_{1}, \ldots, x_{\lambda}, \ldots\right\}$ only for simplicity of writing, since with the axiom of choice we can well-order any set $X, \lambda<\kappa$, for some ordinal $\kappa$, and obtain such notation. It is clear that the definition of a skand does not depend on orderings of elements of $X_{\alpha^{\prime}}, \alpha_{0} \leq \alpha^{\prime}<\alpha$, which may be different, or notations, which may also be alternative. Thus, an arbitrary skand is "only an accidental aggregation" of well-founded sets or individuals each of which figuratively "skips" into its own place, $\{\{$ or \{\} , in the system of well-ordered embedded curly braces; meanwhile there can be empty places as well as a well-founded set, or an individual, and can "skip" into different places and even be at all places at once; i.e., elements of each component are always different and at the same time it may happen that some or even all elements of different components may be equal.

Remark 2. Now we want to dispose of possible objections from the side of some logicians who might say that "braces are not objects of Set Theory and hence, e.g., a trivial skand $\mathbf{e}_{\left(\alpha_{0}, \alpha\right)}$ is not well-defined if $\alpha-$ $\alpha_{0}>\omega$ ", or "an expression such as $\{\ldots\{\{\{\emptyset\}\}\} \ldots\}$ is not definite", or the following, which is thoroughly snobbish: "It would be better if set theory teachers (and books on set theory) said at the outset that it is essential to view the universe of sets as a container intended to contain boxes intended for other boxes and one of them is intended to remain empty. Of course the setbrackets $\{\ldots\}$ suggest this view of sets, but this notion should be explained to those students who meet it for the first time" [48], p. 534 .

However we say that all "pure, well-founded sets" are systems of embedded curly braces; an expression $\{\ldots\{\{\{\emptyset\}\}\} \ldots\}$ is not definite, indeed, but another expression $\{\{\{\ldots\{\emptyset\} \ldots\}\}\}$ of infinite pairs of braces is definite.

In any case the meaning of pairs of braces in the definition of skand is similar as in the definition of "pure well-founded sets" arising from the empty set $\emptyset=\{\}$ :

$$
\{\},\{\{\}\},\{\{\},\{\{\}\}\},\{\{\},\{\{\}\},\{\{\},\{\{\}\}\}\}, \ldots
$$

and we use them only from a most possible convnience outward shape of skand and an attempt to make it visualize.

Actually we can express a skand without any pairs of braces or brackets using only the binary relation $\in$. For this purpose we recall the definition of parametric family of sets [12]), resume, 2, 14. In our case it is a map $f_{\left(\alpha_{0}, \alpha\right)}:\left(\alpha_{0}, \alpha\right) \rightarrow \mathbf{V}[\mathcal{U}], 0 \leq \alpha_{0} \leq \alpha, \alpha_{0}, \alpha \in \mathbf{O n}$, or more precisely, a 
discrete union $\bigsqcup_{\alpha^{\prime} \in\left(\alpha_{0}, \alpha\right)} Y_{\alpha^{\prime}}$, where $Y_{\alpha^{\prime}}$ is a singleton whose the only element is $f_{\left(\alpha_{0}, \alpha\right)}\left(\alpha^{\prime}\right)$, i.e., $f_{\left(\alpha_{0}, \alpha\right)}\left(\alpha^{\prime}\right) \in Y_{\alpha^{\prime}}, \alpha_{0} \leq \alpha^{\prime}<\alpha$.

In our case $\left(\alpha_{0}, \alpha\right)$ is a set of parameters and elements of a parametric sets are elements of $\bigsqcup_{\alpha^{\prime} \in\left(\alpha_{0}, \alpha\right)} Y_{\alpha^{\prime}}$ and equal to $f_{\left(\alpha_{0}, \alpha\right)}\left(\alpha^{\prime}\right) \in \mathbf{V}[\mathcal{U}], \alpha_{0} \leq \alpha^{\prime}<$ $\alpha$.

Now for an arbitrary skand $X_{\left(\alpha_{0}, \alpha\right)}$ one can definitely associate a map $f_{\left(\alpha_{0}, \alpha\right)}:\left(\alpha_{0}, \alpha\right) \rightarrow \mathbf{V}[\mathcal{U}]$ such that for each $\alpha^{\prime}, \alpha_{0} \leq \alpha^{\prime}<\alpha, f_{\left(\alpha_{0}, \alpha\right)}\left(\alpha^{\prime}\right)=$ $X_{\alpha^{\prime}}=\left\{x_{1}^{\alpha^{\prime}}, x_{2}^{\alpha^{\prime}}, \ldots\right\} \in \mathbf{V}[\mathcal{U}]$; if a skand $\mathbf{e}_{\left(\alpha_{0}, \alpha\right)}$ is the empty skand, then the associated map $f_{\left(\alpha_{0}, \alpha\right)}$ is constant and its image is equal to $\emptyset \in \mathbf{V}[\mathcal{U}$.

Conversely, for an arbitrary map $f_{\left(\alpha_{0}, \alpha\right)}:\left(\alpha_{0}, \alpha\right) \rightarrow \mathbf{V}[\mathcal{U}]$ we can associate a unique $X_{\left(\alpha_{0}, \alpha\right)}$ such that associated map coincides with $f_{\left(\alpha_{0}, \alpha\right)}$ : $\left(\alpha_{0}, \alpha\right) \rightarrow \mathbf{V}[\mathcal{U}]$

Indeed, let $f_{\left(\alpha_{0}, \alpha\right)}:\left(\alpha_{0}, \alpha\right) \rightarrow \mathbf{V}[\mathcal{U}]$ be a map. We shall construct a unique skand $X_{\left(\alpha_{0}, \alpha\right)}$ such that the associated map as above will coincide with assumed map $f_{\left(\alpha_{0}, \alpha\right)}:\left(\alpha_{0}, \alpha\right) \rightarrow \mathbf{V}[\mathcal{U}]$. The construction will be done by transfinite induction. Consider the value $f_{\left(\alpha_{0}, \alpha\right)}\left(\alpha_{0}\right) \in \mathbf{V}[\mathcal{U}]$ of $f_{\left(\alpha_{0}, \alpha\right)}$ of its first argument $\alpha_{0} \in\left(\alpha_{0}, \alpha\right)$ and the restriction $f_{\left(\alpha_{0}+1, \alpha\right)}=$ $\left.f_{\left(\alpha_{0}, \alpha\right)}\right|_{\left(\alpha_{0}+1, \alpha\right)}$ of $f_{\left(\alpha_{0}, \alpha\right)}$ on the subset $\left(\alpha_{0}+1, \alpha\right)$ of $\left(\alpha_{0}, \alpha\right)$. We define now a set $X_{(0)}$ whose elements are all elements of the set $f_{\left(\alpha_{0}, \alpha\right)}\left(\alpha_{0}\right) \in \mathbf{V}[\mathcal{U}]$ and the map $f_{\left(\alpha_{0}+1, \alpha\right)}$. More precisely, $X_{(0)}$ is a discrete sum of the set $f_{\left(\alpha_{0}, \alpha\right)}\left(\alpha_{0}\right)$ and the singleton $Y_{(0)} \ni f_{\left(\alpha_{0}+1, \alpha\right)}$, i.e. $X_{(0)}=f_{\left(\alpha_{0}, \alpha\right)}\left(\alpha_{0}\right) \sqcup Y_{(0)}$. It is clear that a new set $X_{(0)}$ is nothing else than an initial map (function) $f_{\left(\alpha_{0}, \alpha\right)}:\left(\alpha_{0}, \alpha\right) \rightarrow \mathbf{V}[\mathcal{U}]$ because it uniquely reconstructs the map $f_{\left(\alpha_{0}, \alpha\right)}$ by putting $f_{\left(\alpha_{0}, \alpha\right)}\left(\alpha_{0}\right)=X_{(0)} \backslash Y_{(0)}$ and $f_{\left(\alpha_{0}, \alpha\right)}\left(\alpha^{\prime}\right)=f_{\left(\alpha_{0}+1, \alpha\right)}\left(\alpha^{\prime}\right), \alpha_{0}+1 \leq$ $\alpha^{\prime}<\alpha$.

(Note that a map or function is a special "functional relation" which can be realized, written, denoted by in different ways like "a graph of function", "implicit function", etc., in our case of well-ordered domain $\left(\alpha_{0}, \alpha\right)$ it is denoted by the set with elements of the image of its first argument and an element which is the restriction of $f_{\left(\alpha_{0}, \alpha\right)}:\left(\alpha_{0}, \alpha\right) \rightarrow \mathbf{V}[\mathcal{U}]$ on $\left.\left(\alpha_{0}, \alpha\right)\right)$, i.e. the map $f_{\left(\alpha_{0}+1, \alpha\right)}:\left(\alpha_{0}+1, \alpha\right) \rightarrow \mathbf{V}[\mathcal{U}]$. In all realizations, representations or designations of $f_{\left(\alpha^{\prime}, \alpha\right)}$ by a graph of function, an implicit function or as in our case by a special recursive set $X_{\left(\alpha^{\prime}\right)}, \alpha_{0} \leq \alpha^{\prime}<\alpha_{0}+\omega$, we speak about the only mathematical object: a map or function $f_{\left(\alpha^{\prime}, \alpha\right)}:\left(\alpha^{\prime}, \alpha\right) \rightarrow \mathbf{V}[\mathcal{U}]$.)

In the same manner we define a unique set $X_{(1)}=X_{\alpha+1} \sqcup Y_{(1)}$, where $Y_{(1)} \ni\left\{f_{(\alpha+2, \alpha)}\right\}$ which uniquely reconstructs the map $f_{\left(\alpha_{0}+1, \alpha\right)}$ and actually equal to it. We continue the construction for all $\alpha^{\prime}$, i.e., change the map $f_{\left(\alpha^{\prime}, \alpha\right)}$ by the set $f_{\left(\alpha^{\prime}, \alpha\right.}\left(\alpha^{\prime}\right) \sqcup Y_{\left(\alpha^{\prime}\right)}, Y_{\left(\alpha^{\prime}\right)} \ni f_{\left(\alpha^{\prime}+1, \alpha\right.}, \alpha_{0} \leq \alpha^{\prime}<\omega \leq \alpha$. We obtain an extraordinary set $X=X_{(0)} \ni X_{(1)} \ni X_{(2)} \ni \ldots$ in the sense 
of Mirimanoff:

$$
X=\left\{x_{1}^{\alpha_{0}}, x_{2}^{\alpha_{0}}, \ldots,\left\{x_{1}^{\alpha_{0}+1}, x_{2}^{\alpha_{0}+1}, \ldots,\left\{\ldots\left\{x_{1}^{\alpha^{\prime}}, x_{2}^{\alpha^{\prime}}, \ldots\right\} \ldots\right\}\right\},\right.
$$

$\alpha_{0} \leq \alpha^{\prime}<\omega \leq \alpha$.

If $\alpha \leq \alpha_{0}+\omega$, then we stop our construction, and this Mirimanoff extraordinary set is nothing else than the initial skand $X_{\left(\alpha_{0}, \alpha\right)}$. But if $\alpha_{0}+$ $\omega<\alpha$, then in formula (6) we see only visible components and parameters of the initial function $f_{\left(\alpha_{0}, \alpha\right)}:\left(\alpha_{0}, \alpha\right) \rightarrow \mathbf{V}[\mathcal{U}]: f_{\left(\alpha_{0}, \alpha\right)}\left(\alpha^{\prime}\right), \alpha^{\prime} \in\left(\alpha_{0}, \alpha_{0}+\omega\right)$ and $\left(\alpha_{0}, \alpha_{0}+\omega\right)$, respectively. And we do not see in (6) other hidden components and parameters of the initial function: $f_{\left(\alpha_{0}+\omega, \alpha\right)}\left(\alpha^{\prime}\right), \alpha_{0}+\omega \leq$ $\alpha^{\prime}<\alpha$, and $\left(\alpha_{0}+\omega, \alpha\right)$, respectively.

We remember that each set $X_{(n)}, 0 \leq n<\omega$, is actually a map $f_{\left(\alpha_{0}+n, \alpha\right)}$, and the latter is a pasting map $f_{\left(\alpha_{0}+n, \alpha\right)}=f_{\left(\alpha_{0}+n, \alpha_{0}+\omega\right)} \cup f_{\left(\alpha_{0}+\omega, \alpha\right)}$. Thus, we have to add in (6) our description of $f_{\left(\alpha_{0}+\omega, \alpha\right)}$ by Mirimanoff sets $X_{(\omega)} \ni$ $X_{(\omega+1)} \ni \ldots \ni X_{\left(\omega_{n}\right)} \ni \ldots, 0 \leq n<\omega$, and so on ... up to the exhaustion of all components $f_{\left(\alpha_{0}, \alpha\right)}\left(\alpha^{\prime}\right), \alpha_{0} \leq \alpha^{\prime}<\alpha$, and all parameters $\left(\alpha_{0}, \alpha\right)$. Thus, we obtain the need skand $X_{\left(\alpha_{0}, \alpha\right)}$ in (5) with $X_{\alpha^{\prime}}=f_{\left(\alpha_{0}, \alpha\right)}\left(\alpha^{\prime}\right), \alpha_{0} \leq \alpha^{\prime}<\alpha$.

Remark 3. One can consider a rigid version of skand theory requiring $\varphi$ in Definition 2 to be an identical isomorphism. This skand theory can naturally describe the "world of non-well-founded sets" given in [19], Chap. II, $\S 5$, which was "supposed to be of a highly artificial nature". The formal symbols $x_{1}, x_{2}, \ldots$ were considered; and formally it was put that $x_{n+1} \in x_{n}$ and $\neg x_{i} \in x_{j}, i \neq j+1$. Putting $R(0)=\left\{x_{1}, x_{2}, \ldots\right\}$, it was defined by recursion $R(\alpha)$ as a power-set of the set $\bigcup_{\beta<\alpha} R(\beta)$. For elements of the set $R(\alpha)$ there was the following $\in$-relation: if $u$ is a set, then $v \in u$, if $v$ is an element of $u$; if $u=x_{i}$, then $v \in u$, if $v=x_{i+1}$. Notice that one needs in the above construction taken in [19] to identify $x_{i}$ with $\left\{x_{i+1}\right\}$, $i \geq 1$. This abstract and formal construction can be naturally described via rigid skands as follows: put $x_{1}=X_{(1, \alpha)}$ with $X_{\alpha^{\prime}}=\emptyset, 1 \leq \alpha^{\prime}<\alpha$, $\alpha \geq \omega$; then $x_{i}=X_{(i, \alpha)}$ are restrictions of $X_{(1, \alpha)}$ on $(i, \alpha), 1<i<\omega$. And then form worlds of non-well-founded sets $R(\alpha), \alpha \geq 1$, as above. We will not consider rigid skands in this paper because they do not allow us to investigate self-similar and reflexive sets.

\section{Example 1.}

$X_{\left(\alpha_{0}, \alpha\right)}=\{\{1,\{2,\{3,\{\ldots\}\}\}\}\}, Y_{\left(\alpha_{0}, \alpha\right)}=\{1,\{\{2,\{3,\{\ldots\}\}\}\}\}$.

Skands $X_{\left(\alpha_{0}, \alpha\right)}$ and $Y_{\left(\alpha_{0}, \alpha\right)}$ are not equal because the $\alpha_{0}$-component $X_{\alpha_{0}}$ of $X_{\left(\alpha_{0}, \alpha\right)}$ is empty and the $\alpha_{0}$-component $Y_{\alpha_{0}}$ of $Y_{\left(\alpha_{0}, \alpha\right)}$ consists of one element, which is equal to 1 . These skands have the same components, but in different order. 
Remark 4. We shall postulate below that an arbitrary skand $X_{\left(\alpha_{0}, \alpha\right)}=$ $\left\{x_{0}^{0}, x_{1}^{0}, \ldots, x_{\lambda_{0}}^{0}, \ldots,\left\{x_{0}^{1}, x_{1}^{1}, \ldots, x_{\lambda_{1}}^{1}, \ldots,\{\ldots\}\right\}\right\}$ is considered as a set in $\mathbf{V}[\mathcal{U}]^{-}$ whose elements are sets or individuals $x_{0}^{0}, x_{1}^{0}, \ldots, x_{\lambda}^{0}, \ldots$ of $\mathbf{U}$ and the skand $X_{\left(\alpha_{0}+1, \alpha\right)}=\left\{x_{0}^{1}, x_{1}^{1}, \ldots, x_{\lambda_{1}}^{1}, \ldots,\{\ldots\}\right\} ;$

i.e., $X_{\left(\alpha_{0}, \alpha\right)}=\left\{x_{0}^{0}, x_{1}^{0}, \ldots, x_{\lambda_{0}}^{0}, \ldots, X_{\left(\alpha_{0}+1, \alpha\right)}\right\}$. In particular, for $l \geq \omega$ a trivial skand $\mathbf{e}_{\left(\alpha_{0}, \alpha\right)}$ can be considered as a set whose only element is this set itself, i.e., $\mathbf{e}_{\left(\alpha_{0}, \alpha\right)}=\left\{\mathbf{e}_{\left(\alpha_{0}, \alpha\right)}\right\}$ because, by Definition $2, \mathbf{e}_{\left(\alpha_{0}, \alpha\right)}=\mathbf{e}_{\left(\alpha_{0}+1, \alpha\right)}$. So, Russell was not right saying that " $X=\{X\}$ must be absolutely meaningless, not simply false".

Clearly, if $\alpha=\alpha_{0}+n$, where $n=1,2, \ldots$ are natural numbers, then

$$
\begin{gathered}
X_{\left(\alpha_{0}, \alpha\right)}=\left\{x_{0}^{0}, x_{1}^{0}, \ldots, x_{\lambda_{0}}^{0}, \ldots,\left\{x_{0}^{1}, x_{1}^{1}, \ldots, x_{\lambda_{1}}^{1}, \ldots\right.\right. \\
\left.\left.\ldots,\left\{\ldots,\left\{x_{0}^{n-1}, x_{1}^{n-1}, \ldots, x_{\lambda_{n-1}}^{n-1}, \ldots\right\}\right\}\right\}\right\}
\end{gathered}
$$

is an ordinary well-founded set whose elements are sets or individuals $x_{0}^{0}, x_{1}^{0}, \ldots, x_{\lambda_{0}}^{0}, \ldots, X_{\left(\alpha_{0}+1, \alpha\right)}$ of $\mathbf{U}$.

Example 2. $X_{(0, \omega)}=\left\{a_{0},\left\{a_{1},\left\{a_{2},\{\ldots\}\right\}\right\}\right\}$, where $a_{i}, i=0,1,2, \ldots$, are individuals or sets in $\mathbf{U}$, where $\omega=\omega_{0}$ is the first infinite ordinal.

This is a skand of length $\omega$ or a two-element set in $\mathbf{V}^{-}$; i.e., $X=$ $\left\{a_{0}, X_{(1, \omega)}\right\}$, where $X_{(1, \omega)}=\left\{a_{1},\left\{a_{2},\{\ldots\}\right\}\right\}$. It is a typical example of a non-well-founded set, or an extraordinary set in the sense of Mirimanoff [43], [44], [45], where $a_{i}, i=0,1,2, \ldots$, are individuals; e.g., non-negative integers $0,1,2, \ldots$; i.e. $X_{(0, \omega)}=\{0,\{1,\{2,\{\ldots\}\}\}\}$, or examples of Mirimanoff's circular extraordinary sets of period $n$, where $a_{i}=a_{i+n}, i=0,1,2, \ldots$, and $n=1,2, \ldots$ is a fixed natural number; e.g., $X_{(0, \omega)}=\{0,\{1,\{0,\{1,\{\ldots\}\}\}\}\}$, where $n=2, a_{0}=0$ and $a_{1}=1$.

The following is a more general example of a circular set.

Example 3. Consider

$$
\begin{array}{r}
X_{(0, \omega)}=\left\{a_{0}, a_{1}, \ldots, a_{\lambda}, \ldots,\left\{b_{0}, b_{1}, . . b_{\mu}, \ldots,\left\{a_{0}, a_{1}, . . a_{\lambda}, \ldots\right.\right.\right. \\
\left.\left.\ldots,\left\{b_{0}, b_{1}, \ldots, b_{\mu}, \ldots,\{\ldots\}\right\}\right\}\right\},
\end{array}
$$

where $a_{\lambda}$ and $b_{\mu}$ are individuals or sets in $\mathbf{U}$ such that $\left\{a_{0}, a_{1}, \ldots, a_{\lambda}, \ldots\right\}$ and $\left\{b_{0}, b_{1}, . . b_{\mu}, \ldots\right\}$ are elements of $\mathbf{V}[\mathcal{U}], 0 \leq \lambda \leq \kappa$ and $0 \leq \mu \leq \nu$, respectively. In other words, an even component $X_{2 n}$ is the set $\left\{a_{0}, a_{1}, \ldots, a_{\lambda}, \ldots\right\}$ and an odd component $X_{2 n+1}$ is the set $\left\{b_{0}, b_{1}, \ldots, b_{\mu}, \ldots\right\}, 0 \leq n<\omega$.

This skand can be considered as circular sets, i.e., $X_{(0, \omega)} \ni X_{(1, \omega)} \ni$ $X_{(0, \omega)}$ and $X_{(1, \omega)} \ni X_{(0, \omega)} \ni X_{(1, \omega)}$ because $X_{(0, \omega)}=\left\{a_{0}, a_{1}, . . a_{\lambda}, \ldots, X_{(1, \omega)}\right\}$, $X_{(1, \omega)}=\left\{b_{0}, b_{1}, . . b_{\mu}, \ldots, X_{(2, \omega)}\right\}, X_{(2, \omega)}=\left\{a_{0}, a_{1}, . . a_{\lambda}, \ldots, X_{(3, \omega)}\right\}$ and, by Definition 2, $X_{(0, \omega)}=X_{(2, \omega)}$ and $X_{(1, \omega)}=X_{(3, \omega)}$.

Example 4. Consider two skands

$$
\begin{aligned}
X_{(0, \omega 2)}= & \left\{a_{0}, a_{1}, \ldots, a_{\lambda}, \ldots,\left\{b_{0}, b_{1}, \ldots, b_{\mu}, \ldots,\left\{a_{0}, a_{1}, . . a_{\lambda}, \ldots\right.\right.\right. \\
& \left.\left.\left.\left\{a_{0}, a_{1}, \ldots, a_{\lambda}, \ldots,\left\{b_{0}, b_{1}, . . b_{\mu},\{\ldots\}\right\}\right\} \ldots\right\}\right\}\right\}
\end{aligned}
$$


where $a_{\lambda}$ and $b_{\mu}$ are individuals or sets in $\mathbf{U}, 0 \leq \lambda \leq \kappa$ and $0 \leq \mu \leq$ $\nu$, respectively, where even components $X_{2 \tau}$ are equal to the fixed set $\left\{a_{0}, a_{1}, \ldots, a_{\lambda}, \ldots\right\}$ and odd components $X_{2 \tau+1}$ are equal to the other fixed set $\left\{b_{0}, b_{1}, \ldots, b_{\mu}, \ldots\right\}, 0 \leq \tau<\omega 2$, and

$$
\begin{aligned}
Y_{(0, \omega 2)}= & \left\{a_{0}, a_{1}, \ldots, a_{\lambda}, \ldots,\left\{b_{0}, b_{1}, \ldots, b_{\mu}, \ldots,\left\{a_{0}, a_{1}, . . a_{\lambda}, \ldots\right.\right.\right. \\
& \left.\left.\left.\left\{b_{0}, b_{1}, \ldots, b_{\mu},\left\{a_{0}, a_{1}, . . a_{\lambda},\{\ldots\}\right\}\right\} \ldots\right\}\right\}\right\}
\end{aligned}
$$

which can be obtained from $X_{(0, \omega 2)}$ by changing $2 \tau$-components $X_{2 \tau}$ on the set $\left\{b_{0}, b_{1}, \ldots, b_{\mu}, \ldots\right\}$ and $2 \tau+1$-components $X_{2 \tau+1}$ on the set $\left\{a_{0}, a_{1}, \ldots, a_{\lambda}, \ldots\right\}$, for all $\omega \leq \tau<\omega 2$.

They can also be considered as circular sets; i.e., $X_{(0, \omega 2)} \ni X_{(1, \omega 2)} \ni$ $X_{(0, \omega 2)}$ and $X_{(1, \omega 2)} \ni X_{(0, \omega)} \ni X_{(1, \omega)}$, because

$X_{(0, \omega 2)}=\left\{a_{0}, a_{1}, . . a_{\lambda}, \ldots, X_{(1, \omega 2)}\right\}, X_{(1, \omega 2)}=\left\{b_{0}, b_{1}, . . b_{\mu}, \ldots, X_{(2, \omega 2)}\right\}$,

$X_{(2, \omega 2)}=\left\{a_{0}, a_{1}, . . a_{\lambda}, \ldots, X_{(3, \omega 2)}\right\}$ and, by Definition $2, X_{(0, \omega 2)}=X_{(2, \omega 2)}$ and $X_{(1, \omega 2)}=X_{(3, \omega 2)}$ as well as $Y_{(0, \omega 2)} \ni Y_{(1, \omega 2)} \ni Y_{(0, \omega 2)}$ and $Y_{(1, \omega 2)} \ni$ $Y_{(0, \omega 2)} \ni Y_{(1, \omega 2)}$, because

$Y_{(0, \omega 2)}=\left\{a_{0}, a_{1}, . . a_{\lambda}, \ldots, Y_{(1, \omega 2)}\right\}, Y_{(1, \omega 2)}=\left\{b_{0}, b_{1}, . . b_{\mu}, \ldots, Y_{(2, \omega 2)}\right\}, Y_{(2, \omega 2)}=$ $\left\{a_{0}, a_{1}, . . a_{\lambda}, \ldots, Y_{(3, \omega 2)}\right\}$ and, by Definition $2, Y_{(0, \omega 2)}=Y_{(2, \omega 2)}$ and $Y_{(1, \omega 2)}=$ $Y_{(3, \omega 2)}$. Nevertheless, $X_{(0, \omega 2)} \neq Y_{(0, \omega 2)}$, as well as $X_{(1, \omega 2)} \neq Y_{(1, \omega 2)}$. It is also clear that $X_{(0, \omega)} \neq X_{(0, \omega 2)}$ and $X_{(0, \omega)} \neq Y_{(0, \omega 2)}$.

Note that every well-founded set $X=\left\{x_{0}, x_{1}, \ldots, x_{\lambda}, \ldots\right\}$ can be considered not only as a skand $X_{(0,1)}$ of length 1 but also as many other different skands of different finite lengths in general. Indeed, fix, for example, one element of $X$. Let it be $x_{0}$. Since $X$ is well-founded we choose a descending $\in$-chain $x_{0} \ni x_{0}^{1} \ni x_{0}^{2} \ni \ldots \ni x_{0}^{n}$ such that $x_{0}^{n}$ is an individual or the empty set, and fix it. Then $X=X_{(0, n+1)}=\left\{x_{1}, x_{2}, \ldots, x_{\lambda}, \ldots, x_{0}\right\}$, where $x_{0}=\left\{a_{1}^{1}, a_{2}^{1}, \ldots, a_{\lambda_{1}}^{1}, \ldots, a_{0}^{1}\right\}$ and $a_{0}^{1}=x_{0}^{1}=\left\{a_{1}^{2}, a_{2}^{2}, \ldots, a_{\lambda_{2}}^{2}, \ldots, a_{0}^{2}\right\}$, $a_{0}^{2}=x_{0}^{2}=\left\{a_{1}^{3}, a_{2}^{3}, \ldots, a_{\lambda_{3}}^{3}, \ldots, a_{0}^{3}\right\}, \ldots, a_{0}^{n-1}=x_{0}^{n-1}=\left\{a_{1}^{n}, a_{2}^{n}, \ldots, a_{\lambda_{n}}^{n}, a_{0}^{n}\right\}$, $a_{0}^{n}=x_{0}^{n}$; i.e., $X=X_{(0, n+1)}=\left\{x_{1}, x_{2}, \ldots, x_{\lambda}, \ldots\right.$ $\left.\ldots,\left\{a_{1}^{1}, a_{2}^{1}, \ldots, a_{\lambda_{1}}^{1}, \ldots,\left\{\ldots,\left\{a_{1}^{n}, a_{2}^{n}, \ldots, a_{\lambda_{n}}^{n}, \ldots, a_{0}^{n}\right\}\right\}\right\}\right\}$.

If for example, $x_{0}^{n}=\emptyset$, then there is another skand which gives the same set $X=X_{(0, n+2)}=\left\{x_{1}, x_{2}, \ldots, x_{\lambda}, \ldots,\left\{a_{1}^{1}, a_{2}^{1}, \ldots, a_{\lambda_{1}}^{1}, \ldots,\{\ldots\right.\right.$ $\left.\left.\left.\ldots,\left\{a_{1}^{n}, a_{2}^{n}, \ldots, a_{\lambda_{n}}^{n}, \ldots,\{\}\right\}\right\}\right\}\right\}$.

Example 5. $X_{(0, \omega+1)}=\left\{a_{0},\left\{a_{1},\left\{a_{2},\left\{\ldots\left\{a_{\omega}\right\} \ldots\right\}\right\}\right\}\right.$, where $a_{i}, i=$ $0,1,2, \ldots, \omega$, are individuals or sets in $\mathbf{U}$.

This is the simplest example of non-well-founded set whose only two elements are $a_{0}$ and skand $X_{(1, \omega+1)}=\left\{a_{1},\left\{a_{2},\left\{\ldots\left\{a_{\omega}\right\} \ldots\right\}\right\}\right.$. It was not considered by Mirimanoff because by remaining silent on the issue of an extraordinary set, i.e., $X \ni X_{1} \ni X_{2} \ni$..., Mirimanoff seems to suggest 
that it is in our terminology a skand of length $\omega$ only. Moreover, all such skands and others of length $\omega+\beta$, where $\beta \in \mathbf{O n}, \beta \geq 1$, are here essential generalizations and extensions of some of Mirimanoff's extraordinary sets.

3. The formal theory $N B G[\mathcal{U}]^{(1)}$ of non-well-founded sets and its model in the universe of elements $\mathbf{U}[\mathcal{U}]^{(1)}$

Recall that we work here inside $N B G^{-}$and $N B G[\mathcal{U}]^{-}$set theories, where the former is the theory of "pure" sets and latter is the theory with individuals $\mathcal{U}$, and in both theories the Axiom of Foundation FA has been omitted. As above, by $\mathbf{V}^{-}$and $\mathbf{V}[\mathcal{U}]^{-}$we denote the classes of sets in these theories, as well as the universes of elements $\mathbf{U}^{-}=\mathbf{V}^{-}$and $\mathbf{U}[\mathcal{U}]^{-}=$ $\mathbf{V}^{-} \cup \mathcal{U}$, respectively. Similar notations are for their extensions as the same set theories plus the Axiom of Foundation FA: $N B G$ and $N B G[\mathcal{U}]$ set theories, where the former is the theory of "pure" sets and latter is the theory with individuals $\mathcal{U}, \mathbf{V}$ and $\mathbf{V}[\mathcal{U}]$ are the classes of sets in these theories, as well as the universes of elements $\mathbf{U}=\mathbf{V}$ and $\mathbf{U}[\mathcal{U}]=\mathbf{V} \cup \mathcal{U}$, respectively.

We want to extend $N B G^{-}$and $N B G[\mathcal{U}]^{-}$to $N B G^{(1)}$ and $N B G[\mathcal{U}]^{(1)}$, respectively, by less restrictive axioms than FA, which will be based nevertheless on $N B G \stackrel{\text { def }}{=} N B G^{(0)}$. For transfinite inductive constructions we also put $N B G[\mathcal{U}] \stackrel{\text { def }}{=} N B G[\mathcal{U}]^{(0)}, \mathbf{V} \stackrel{\text { def }}{=} \mathbf{V}^{(0)}, \mathbf{V}[\mathcal{U}] \stackrel{\text { def }}{=} \mathbf{V}[\mathcal{U}]^{(0)}, \mathbf{U} \stackrel{\text { def }}{=} \mathbf{U}^{(0)}$ $\mathbf{U}[\mathcal{U}] \stackrel{\text { def }}{=} \mathbf{U}[\mathcal{U}]^{(0)}$, and $\mathcal{U}^{\text {def }}=\mathcal{U}^{(0)}$, respectively.

Suppose now that $\mathcal{U}^{(0)}$ is a set (which can be empty) or class and we will define a theory $N B G[\mathcal{U}]^{(1)}$ which extend in these different cases $N B G^{-}$ and $N B G[\mathcal{U}]^{-}$, turning our attention to the latter because the former is a particular case of $N B G[\mathcal{U}]^{-}$when $\mathcal{U}=\emptyset$. For this purpose we add to $N B G^{(0)}$ a wider class of individuals than $\mathcal{U}^{(0)}$ by adding to it a new class of individuals $\mathcal{U}^{(1)}$. Elements $u_{\lambda}^{(1)} \in \mathcal{U}^{(1)}, \lambda \in$ Ord, are arbitrary skands $X_{\left(\alpha_{0}, \alpha\right)}$ of length $l=\left(\alpha-\alpha_{0}\right) \geq \omega$ taken with the forgetful operator $E$ which "forgets" the inner structure ( $\in$ relations) of $X_{\left(\alpha_{0}, \alpha\right)}$. More precisely, $u_{\lambda}^{(1)}=E X_{\left(\alpha_{0}, \alpha\right)}$ and no element or object is a member of $E X_{\left(\alpha_{0}, \alpha\right)}$. Moreover, if $X_{\left(\alpha_{0}, \alpha\right)}=X_{\left(\beta_{0}, \beta\right)}$, then $E X_{\left(\alpha_{0}, \alpha\right)}=E X_{\left(\beta_{0}, \beta\right)}$ and they define the only individual $u_{\lambda}^{(1)} \in \mathcal{U}^{(1)}$. We will need the inverse operator, i.e., "remember operator" $E^{-1}$, i.e., for any individual $u_{\lambda}^{(1)}=E X_{\left(\alpha_{0}, \alpha\right)}$, $\lambda \in \operatorname{Ord}, E^{-1} u_{\lambda}^{(1)}=X_{\left(\alpha_{0}, \alpha\right)}$.

By $N B G[\mathcal{U}]^{(1)}$ we understand the theory $N B G[\mathcal{U}]^{-}$with the addition axiom: 
Skand Existence Axiom and Recognition Skand as a Set. For any map $f_{\left(\alpha_{0}, \alpha\right)}:\left(\alpha_{0}, \alpha\right) \rightarrow \mathbf{V}[\mathcal{U}], \alpha-\alpha_{0} \geq \omega$, there exists a unique skand $X_{\left(\alpha_{0}, \alpha\right)}$ such that for each $\alpha^{\prime} \in\left(\alpha_{0}, \alpha\right)$ one has $X_{\alpha^{\prime}}=f_{\left(\alpha_{0}, \alpha\right)}\left(\alpha^{\prime}\right)$. This skand is considered as a set $X \in \mathbf{V}[\mathcal{U}]^{-}$whose elements are elements of the set $f_{\left(\alpha_{0}, \alpha\right)}\left(\alpha_{0}\right)$ and one more element $X_{\left(\alpha_{0}+1, \alpha\right)}$ which is a restriction of $X_{\left(\alpha_{0}, \alpha\right)}$ on $\left(\alpha_{0}+1, \alpha\right)$, i.e.,

$$
X=\left\{x_{0}^{\alpha_{0}}, x_{1}^{\alpha_{0}}, \ldots, x_{\lambda}^{\alpha_{0}}, \ldots, X_{\left(\alpha_{0}+1, \alpha\right)}\right\} .
$$

We will note this very set by the same symbol $X_{\left(\alpha_{0}, \alpha\right)}$ but we shall differ meaning $X_{\left(\alpha_{0}, \alpha\right)}$ as a skand (5) and $X_{\left(\alpha_{0}, \alpha\right)}$ as a set (10).

Remark 5. One can notice that we ignore here slands $X_{\left(\alpha_{0}, \alpha\right)}$ of finite length, i.e., $l=\alpha-\alpha_{0}<\omega$, because in this case the set $X_{\left(\alpha_{0}, \alpha\right)}$ is well-founded set and will not enrich $\mathbf{V}[\mathcal{U}]^{(0)}$. When a skand $X_{\left(\alpha_{0}, \alpha\right)}$ has a length $l=\alpha-\alpha_{0} \geq \omega$, then the set $X_{\left(\alpha_{0}, \alpha\right)}$ is a non-well-founded set because by Skand Existence Axiom and Recognition Skand as a Set (shortly SEA\&RSS) in $X_{\left(\alpha_{0}, \alpha\right)}$, repeatting $\omega$ times, in the set $X_{\left(\alpha_{0}, \alpha\right)}$ there is an infinite $\in$-descending chain:

$$
X_{\left(\alpha_{0}, \alpha\right)} \ni X_{\left(\alpha_{0}+1, \alpha\right)} \ni X_{\left(\alpha_{0}+2, \alpha\right)} \ni \ldots \ni X_{\left(\alpha^{\prime}, \alpha\right)} \ni \ldots, \alpha_{0} \leq \alpha^{\prime}<\alpha .
$$

Since in $N B G[\mathcal{U}]^{(1)}$ we postulate existence of new objects and sets we have to revise the previous Extensionality Axiom of $N B G[\mathcal{U}]^{(0)}$.

Strong Extensionality Axiom. Two sets (resp., classes) $X^{(1)}$ and $Y^{(1)}$, whose elements are well-founded sets, individuals and skands as nonwell-founded sets are equal if for each element $x \in X^{(1)}$ there is an element $y \in Y^{(1)}$ such that $x=y$ and for each element $y \in Y^{(1)}$ there is an element $x \in X^{(1)}$ such that $y=x$, where "=" means the following:

1) the equality of individuals, when $x, y \in \mathcal{U}^{(0)}$, i.e., $(x=y) \Longleftrightarrow$ $(\forall z)(x \in z \Longleftrightarrow y \in z)$;

2) the equality of well-founded sets, when $x, y \in \mathbf{V}[\mathcal{U}]^{(0)}$, i.e., by $\mathbf{E A}$ of $N B G[\mathcal{U}]$

3) the equality of skands, when $x, y \in E^{-1} \mathcal{U}^{(1)}$, i.e., by Definition 2 ;

4) the (iterative) equality of sets in $\mathbf{V}[\mathcal{U}]^{(1)}$, i.e., by using 1), 2), 3) in SEA for a complex sets $x, y \in \mathbf{V}[\mathcal{U}]^{(1)}$.

The latter class $\mathbf{V}[\mathcal{U}]^{(1)}$ is defined by transfinite recursion. Denote by $\mathcal{U}^{\prime}$ the discrete union $\mathcal{U}^{(0)} \sqcup \mathcal{U}^{(1)}$. By formulas (2) and (3) we define a class $\mathbf{H}\left[\mathcal{U}^{\prime}\right]$ which is a class of well-founded sets and determines a model of $N B G\left[\mathcal{U}^{\prime}\right], \mathbf{H}\left[\mathcal{U}^{\prime}\right]=\mathbf{U}\left[\mathcal{U}^{\prime}\right]$ and is an inner model of $N B G\left[\mathcal{U}^{\prime}\right]^{-}$. Now we apply the remember operator $E^{-1}$ to all individuals of $\mathcal{U}^{(1)}$ which are in any constituents of sets in $\mathbf{V}\left[\mathcal{U}^{\prime}\right]=\mathbf{H}\left[\mathcal{U}^{\prime}\right] \backslash \mathcal{U}^{(0)}$ or individuals themselves 
in $\mathbf{H}\left[\mathcal{U}^{\prime}\right] \backslash \mathcal{U}^{(0)}$, in short words, in all objects in $X \in \mathbf{H}\left[\mathcal{U}^{\prime}\right] \backslash \mathcal{U}^{(0)}$ we change all individuals of $\mathcal{U}^{(1)}$ on the corresponding skands considered as sets, i.e., each individual $E X_{\left(\alpha_{0} . \alpha\right)}$ turns into a set $X_{\left(\alpha_{0} . \alpha\right)}$.

One can see that after such changing $\mathbf{V}\left[\mathcal{U}^{\prime}\right]$ turns into a class $\mathbf{V}[\mathcal{U}]^{(1)}$ of sets which can be well-founded and not-well-founded. Moreover, by Propositions 1 and 2, V $[\mathcal{U}]^{(1)}$ is a model of $N B G[\mathcal{U}]^{(1)}$ and an inner model of $N B G[\mathcal{U}]^{-}$.

These definitions and construction of $N B G[\mathcal{U}]^{(1)}$ give the following theorem.

Theorem 1. The set theory $N B G[\mathcal{U}]^{(1)}$ is consistent on the assumption that $N B G^{-}$is consistent.

Proof. As we mentioned above, $N B G^{-}$is consistent iff $N B G[\mathcal{U}]^{-}$is consistent. It is well known that if $N B G[\mathcal{U}]^{-}$is consistent, then $N B G[\mathcal{U}]=$ $N B G[\mathcal{U}]^{(0)}$ is also consistent (see Exercise 4.86 in [41]). But $N B G[\mathcal{U}]$ is consistent iff $N B G$ is consistent. Again $N B G$ is consistent iff $N B G\left[\mathcal{U}^{\prime}\right]$ is consistent. But the remember operator $E^{-1}$ makes from $N B G\left[\mathcal{U}^{\prime}\right]$ a consistent theory $N B G[\mathcal{U}]^{(1)}$, too. (See details in [47] or Chap. 4, Proposition 4.50 in [41]).

Remark 6. In our case we have an additional class of individuals $\mathcal{U}^{(1)}$ as "pseudo-individuals" in the above sense, i.e., each $\in$-descending chain is finite or terminates, i.e., reaches its bottom or "foundation" which is an empty set, or an individual, or a skand.

Since $\emptyset \subset \mathcal{U}$ it is clear that by the second part of SEA\&RSS, i.e., RSS, we obtain the following embeddings:

$$
\mathbf{V}[\mathcal{U}]=\mathbf{V}[\mathcal{U}]^{(0)} \subset \mathbf{V}[\mathcal{U}]^{(1)} \subset \mathbf{V}[\mathcal{U}]^{-} .
$$

Examples 3, 4, 5, show that the former embedding is proper; we shall see that the latter embedding is proper, too.

\section{Self-similar skands}

The following skands are of great interest in the study of the relation $X \in X$ in the Set Theory which we are going to clarify in this paper.

Definition 3. A skand $X_{\left(\alpha_{0}, \alpha\right)}$ is called self-similar if for each $\alpha_{1}$, $\alpha_{0} \leq \alpha_{1}<\alpha$, there is an equality $X_{\left(\alpha_{0}, \alpha\right)}=X_{\left(\alpha_{1}, \alpha\right)}$.

It happens iff $\alpha=\omega^{\kappa}$, where $\omega=\omega_{0}$ is the initial countable ordinal, $\kappa \geq 1$, and each $\alpha^{\prime}$-component $X_{\alpha^{\prime}}$ of $X_{\left(\alpha_{0}, \omega^{\kappa}\right)}, \alpha_{0} \leq \alpha^{\prime}<\omega^{\kappa}$, is the same set $\left\{x_{1}, x_{2}, \ldots, x_{\lambda}, \ldots,\{\right.$.

Indeed, it is well known that each remainder $\rho$ of an ordinal $\alpha \neq 0$ is equal to $\alpha$ if and only if $\alpha=\omega^{\kappa}, \kappa \geq 0$, ([36], Ch. VII, $\S 7$, Theorem 7). Recall that an ordinal $\rho$ is called a remainder of an ordinal $\alpha$ if $\rho \neq 0$ and there exists an ordinal $\sigma$ such that $\alpha=\sigma+\rho$. 
Then our assertion when skands are self-similar follows immediately from Definition 2.

Note that in the case $\kappa=0$ the skands $X_{(0,1)}$ which are ordinary wellfounded sets can be formally considered as "self-similar" skands of length 1 .

Recall also that ordinal numbers $\alpha$ of the form $\omega^{\kappa}, \kappa \geq 0$, turn out to be the prime components or principal numbers of addition, i.e., ordinal numbers $\alpha$ such that there is no decomposition $\alpha=\beta+\gamma$ where $\beta<\alpha$ and $\gamma<\alpha([56], \S 19$, Chap. XIV, Theorem 1, p. 323).

Example 6. $X_{(0, \omega)}=\left\{a_{0},\left\{a_{0},\left\{a_{0},\{\ldots\}\right\}\right\}\right.$, where $a_{0}$ is an individual or set in $\mathbf{U}$.

This is a self-similar skand of length $\omega$ or the simplest example of an extraordinary circular set of period 1 .

More generally, $X_{(0, \omega)}=\left\{a_{0}, a_{1}, a_{2}, \ldots, a_{\lambda}, \ldots\left\{a_{0}, a_{1}, a_{2}, \ldots, a_{\lambda}, \ldots\{\ldots\}\right\}\right\}$, where $a_{\lambda}, 0 \leq \lambda<\kappa$, are individuals or sets in $\mathbf{U}$, e.g., for $\lambda=i, a_{\lambda}=i$, $0 \leq i<\omega=\kappa, X_{(0, \omega)}=\{0,1,2,3, \ldots,\{0,1,2,3, \ldots,\{\ldots\}\}\}$.

Example 7. $X_{\left(0, \omega^{2}\right)}=\left\{a_{0},\left\{a_{0},\left\{a_{0},\{\ldots\}\right\}\right\}\right\}$, where $a_{0}$, is an individual or a set in $\mathbf{U}$.

This is an example of a self-similar skand of length $\omega^{2}$. We picture it in the following figure:

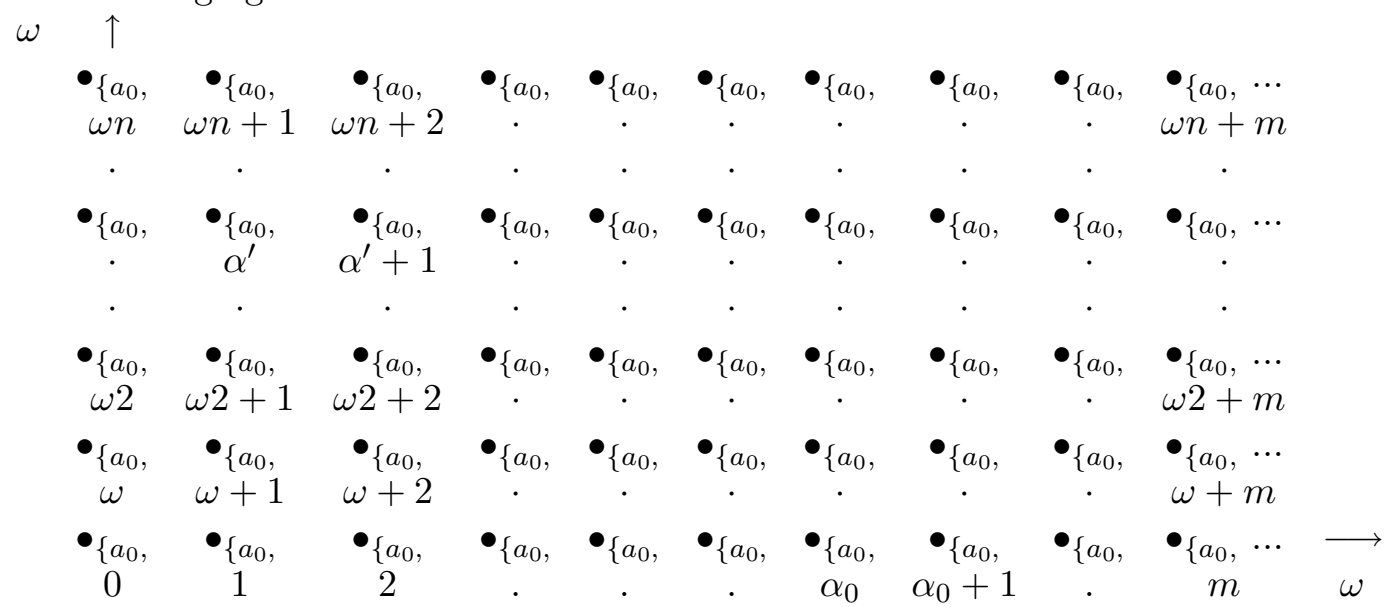

Fig. 1 .

and conclude that $X_{\left(\alpha_{0}, \omega^{2}\right)} \approx X_{\left(\alpha^{\prime}, \omega^{2}\right)}$, for all $0 \leq \alpha_{0}<\alpha^{\prime}<\omega^{2}$; i.e., we see that $X_{\left(0, \omega^{2}\right)}$ is really a self-similar skand of length $\omega^{2}$. Notice also that, by Definition 2, $X_{(0, \omega)}=\left\{a_{0},\left\{a_{0},\left\{a_{0},\{\ldots\}\right\}\right\}\right\} \neq X_{\left(0, \omega^{2}\right)}=$ $\left\{a_{0},\left\{a_{0},\left\{a_{0},\{\ldots\}\right\}\right\}\right\}$.

Definition 4. A set $X$ is called reflexive if $X \in X$. 
It is not clear at once that reflexive sets do exist, in spite of Mirimanoff's "ensembles de deuxième sort" [43] and Eklund's "Mengen, die Elemente ihrer selbst sind" [22].

Consider now an "indeterminate" object $X$ in $\mathbf{V}[\mathcal{U}]^{-}$of the following form:

$$
X=\left\{x_{0}, x_{1}, x_{2}, \ldots, x_{\lambda}, \ldots, X\right\} .
$$

It is clear that the equation (13) is a general form of reflexive sets if they exist.

Proposition 3. Reflexive sets do exist. Moreover, there are a huge number of different solutions (which form a proper class) of (13) in $\mathbf{V}[\mathcal{U}]^{-}$.

Proof. Indeed, the following self-similar skands:

$$
X_{\left(0, \omega^{\kappa}\right)}=\left\{x_{0}, x_{1}, x_{2}, \ldots, x_{\lambda}, \ldots,\left\{x_{0}, x_{1}, x_{2}, \ldots, x_{\lambda}, \ldots,\{\ldots\}\right\}\right\},
$$

for each $\kappa \in \mathbf{O n}, \kappa>0$, are solutions of (13) because, by the Axiom of Skand Existence, objects in the form (14) do exist and by Definition 2, $X_{\left(0, \omega^{\kappa}\right)}=X_{\left(1, \omega^{\kappa}\right)}$, and we obtain

$$
X=X_{\left(0, \omega^{\kappa}\right)}=\left\{x_{0}, x_{1}, x_{2}, \ldots, x_{\lambda}, \ldots, X_{\left(1, \omega^{\kappa}\right)}\right\}=\left\{x_{0}, x_{1}, x_{2}, \ldots, x_{\lambda}, \ldots, X\right\} .
$$

Remark 7. Proposition 3 shows that the relation $X \in X$ is extremely mulivalued and Russell was more or less right to call it "meaningless" because without additional description it is undefined. One can say the same thing about the relations $X \in Y \in X$ which are also many-valued and, without additional description, are undefined, as Examples 3 and 4 tell us.

Remark 8. Many years ago the author noticed (better to say perceived) [38] that there are self-similar skands of length greater than $\omega$ and for a long time has been thinking that only such skands of length $\omega^{\kappa}, \kappa \geq 2$, are solutions of (13) which differ from each other and from the solution of length $\omega$, i.e., Mirimanoff's extraordinary set solution. Now it is clear that not only, e.g.,

$$
X_{(0, \omega)}=\left\{x_{0}, x_{1}, x_{2}, \ldots, x_{\lambda}, \ldots,\left\{x_{0}, x_{1}, x_{2}, \ldots, x_{\lambda}, \ldots,\{\ldots\}\right\}\right\}
$$

and

$$
X_{\left(0, \omega^{2}\right)}=\left\{x_{0}, x_{1}, x_{2}, \ldots, x_{\lambda}, \ldots,\left\{x_{0}, x_{1}, x_{2}, \ldots, x_{\lambda}, \ldots,\{\ldots\}\right\}\right\}
$$

whose components are the same set $X_{\alpha^{\prime}}=\left\{x_{1}, x_{2}, \ldots, x_{\lambda}, \ldots\right\}, 0 \leq \alpha^{\prime}<\omega$ and $0 \leq \alpha^{\prime}<\omega^{2}$, respectively, are different solutions of (13), but also, e.g.,

$$
X_{(0, \omega+1)}=\left\{x_{0}, x_{1}, x_{2}, \ldots, x_{\lambda}, \ldots,\left\{x_{0}, x_{1}, x_{2}, \ldots, x_{\lambda},\{\ldots\{1\} \ldots\}\right\}\right\}
$$

and

$$
Y_{(0, \omega+1)}=\left\{x_{0}, x_{1}, x_{2}, \ldots, x_{\lambda}, \ldots,\left\{x_{0}, x_{1}, x_{2}, \ldots, x_{\lambda},\{\ldots\{2\} \ldots\}\right\}\right\}
$$


are also different solutions of (13) because $X_{(0, \omega+1)}=X_{(1, \omega+1)}$ and $Y_{(0, \omega+1)}=$ $Y_{(1, \omega+1)}$ and $X_{\omega}=\{1\} \neq\{2\}=Y_{\omega}$, although these solutions are not selfsimilar skands. So, reflexive sets need not be self-similar skands. On the other hand, all these different solutions above are isomorphic extraordinary sets in the sense of Mirimanoff [15], p. 40-41, and form a proper class. Moreover, they are identically isomorphic extraordinary sets in Mirimanoff's sense and thus must be equal. So, identically isomorphic extraordinary sets, or equal extraordinary sets need not be equal in $N B G^{(1)}$. In other words, reflexive extraordinary sets in [43] as well as in [22] are not well-defined, if we do not restrict them to skands of length $\omega$. Judging by his silence on the issue, it seems that Mirimanoff tacitly supposed that the length of the skands was equal to $\omega$. Consequently, in Mirimanoff's approach we see only $\omega$-phenomena and ignore trans- $\omega$-phenomena. This is indeed the origin of the following error in logic which we are now going to clarify.

\section{Applications to Russell's paradox and its variants 5.1 Russell's paradox.}

In 1903 Russell published the famous paradox he had discovered two years previously and of which he had informed other mathematicians by correspondence. Here is the original quotation: "We examined the contradiction resulting from the apparent fact that if $w$ be the class of all classes which as single terms are not members of themselves as many, then $w$ as one can be proved both to be and not to be a member of itself as many" ([54], Chap. X, p. 107). Thus, in modern terminology, he defined the following set:

$$
R=\{X \mid X \notin X\}
$$

where $X$ are sets; i.e., $R$ is the set of all sets that are not members of themselves, or the universal set formed by the property $X \notin X$, which he and then all mathematicians considered to be a paradoxical set, or Russell's antinomy. Therefore, the set $R$ was supposed to be inconsistent in Cantor's Naïve Set Theory and the latter was called inconsistent, e.g., [9], p. 488489.

The property or predicate $X \notin X$ was called Russell's condition.

Russell's argument is the following:

$$
R \in R \Longleftrightarrow R \notin R
$$

which is a contradiction, and that is why the set $R$ is inconsistent. Hence, $R$ does not exist from Poincaré's point of view: "En mathématiques le mot exister ne peut avoir qu'un sens: il signifie exempt de contradiction" ([52], 
p. 162), as well as from Russell's: "The contradiction proves that the class as one, if it ever exists, is certainly sometimes absent" ([54], Chap. X, p. 107).

Later on, when sets and proper classes were being distinguished and any predicate (in particular, Russell's condition) formed a class which existed by one of the class-formation axioms, it was supposed that Russell's paradox said nothing other than that $R$ was a proper class, not a set. E.g., an explicit exposition of it we take from [41], p. 239: "Let us verify now that the usual argument for Russell's paradox does not hold in $N B G^{-}$. By the class existence theorem, there is a class $Y=\{x \mid x \notin x\}$. Then $(\forall x)(x \in$ $Y \Longleftrightarrow x \notin x)$. In unabbreviated notion this becomes $(\forall X)(M(X) \Longrightarrow$ $(X \in Y \Longleftrightarrow X \notin X))$. Assume $M(Y)$. Then $Y \in Y \Longleftrightarrow Y \notin Y$, which, by the tautology $(A \Longleftrightarrow \neg A) \Longrightarrow(A \wedge \neg A)$, yields $Y \in Y \wedge Y \notin Y$. Now, by the deduction theorem, we obtain $\vdash M(Y) \Longrightarrow(Y \in Y \wedge Y \notin Y)$, and then, by tautology $(B \Longrightarrow(A \wedge \neg A)) \Longrightarrow \neg B$, i.e., the derived rule of proof by contradiction, we obtain $\vdash \neg M(Y)$. Thus, in $N B G^{-}$, the argument for Russell's paradox merely shows that Russell's class $Y$ is a proper class, not a set. $N B G^{-}$will avoid the paradoxes of Cantor and Burali-Forti in a similar way". (In this citation we have only changed $N B G$ into $N B G^{-}$, which is in our notation the corresponding set theory; $\vdash \mathcal{A}$ means that $\mathcal{A}$ is a theorem.)

Now, by Proposition 3, we can state the following conjecture:

Conjecture 1. In any set theory, if one assumes that $R=\{X \mid M(X) \wedge$ $X \notin X\}$ is a set, then the implication

$$
R \notin R \Longrightarrow R \in R
$$

is false, i.e., Russell's argument (21) is false and hence Russell's paradox is not a paradox in this set theory; thus, the proof that $R$ is a proper class, not a set, via Russell's paradox is incorrect.

The sense of this conjecture is the following: after assuming $M(R)$ we notice that

1) Russell's condition $X \notin X$ becomes impredicative;

2 ) by (13) and (14), the relation $R \in R$ is multi-valued;

3 ) a set $R$ with the relation $R \in R$ (which in this case is reflexive and hence a non-well-founded set) in general is not well-defined, i.e., indefinite.

We shall prove this conjecture for some well-known formal systems (set theories), some from the syntactic and the semantic sides, some only from semantic side.

Proposition 4. Conjecture 1 is true in $N B G$, i.e., if $M(R)$, then the implication $R \notin R \Longrightarrow R \in R$ is false in $N B G$. 
Proof. It is well known that the axiom of regularity implies $(\forall X)(M(X)$ $\Longrightarrow X \notin X)$. Indeed, otherwise, the set $Y=\{X\}$ would not satisfy the axiom of regularity $\operatorname{Reg}$ (4). (See the more general Proposition 4.44(c), p. 279, in [41].) Thus, for each set $X$ the statement (respectively, formula) $X \notin$ $X$ is always true (respectively, true in some and hence in all interpretations or models of $N B G$ ) and the statement $X \in X$ is always false (respectively, false in some, and hence in all interpretations or models of $N B G$ ) in $N B G$. In particular, if $M(R)$, then $R \notin R$ is true and $R \in R$ is false. Hence, by the calculation of the truth function for implication, the following statement (respectively, formula) $R \notin R \Longrightarrow R \in R$ is false (respectively, false in some and hence in all interpretations or models).

Corollary 1. Russell's paradox in $N B G$ is not a paradox which "shows $\operatorname{Pr}(R)$ ", and hence the classical proof that $R$, which in $N B G$ coincides with $\mathbf{V}[\mathcal{U}]$, is a proper class is not correct when it proceeds via Russell's paradox.

Proof. Since by Proposition 4, the implication $R \notin R \Longrightarrow R \in R$ is false in $N B G$ we obtain that there is no equivalence (21) and hence the above argument $\vdash M(R) \Longrightarrow(R \in R \wedge R \notin R)$ and, that by the derived rule of proof by contradiction, one obtains $\vdash \neg M(R)$, does not work any more. Thus, in $N B G \vdash \neg M(R)$ proved via Russell's paradox is incorrect.

Nevertheless, the latter $\vdash \neg M(R)$ can be easily proved by the Maximality Principle.

Proposition 5. Russell's collection $R=\mathbf{V}[\mathcal{U}]$ in $N B G$ is a proper class, not a set.

Proof. Since, by the axiom of regularity, for each set $X \in \mathbf{V}[\mathcal{U}]$ the statement $X \notin X$ is true, we obtain the relation $X \in R$. Thus, $R=\mathbf{V}[\mathcal{U}]$. Assume that $M(R)$. Then, as above, the statement $R \notin R$ is true. By the Axiom of Pairing, i.e., for every two sets $X, Y$ there exists a set $\{X, Y\}$ that has $X$ and $Y$ as its only members, in particular, when $X=Y$, there exists a singleton $\{X\}$, thus, we obtain the singleton $\{R\} \in \mathbf{V}[\mathcal{U}]$. Now, by the Axiom of Union, we obtain the set $R \cup\{R\}$ with a proper subset $R$, i.e., $R \subset R \cup\{R\}$, which is in contradiction with the maximality of $R$, since it is the set of all elements $X$ such that $X \notin X$; but by assertion $M(R)$, $R \notin R$. This proves the statement $\neg M(R)$, i.e., $\operatorname{Pr}(R)$.

Remark 9. In proofs of Propositions 4 and 5 we avoid the traditional (e.g., [41], Chap. 4, §1, [36], [12], [27], [4], etc.) arguments: Assuming $M(R)$, by the derived rule of proof by definition (actually, by a hidden axiom) and that $R \in R$ is a well-formed formula, the statement $R \notin R \Longrightarrow$ $R \in R$ is supposed to be true. Indeed, the formula $R \in R$ is well-formed, but in general a set $R$ with the relation $R \in R$ is not well-defined, because a reflexive set $R$, i.e., $R \in R$, has a non-definite element $R$ and we cannot say 
what set we are dealing with; moreover, if $R$ is well-defined in some formal systems, the statement $R \in R$ can be false, as we have seen in the above proofs. If the statement $R \notin R \Longrightarrow R \in R$ were true by the definition of $R$ and a well-formed formula $R \in R$ or a well-defined non-well-founded set $R$, there should be a double-paradox of Russell. Indeed, in $N B G$ there should be two opposite statements: $R \notin R \Longrightarrow R \in R$ is true and $R \notin R \Longrightarrow$ $R \in R$ is false. Since the statement $R \in R \Longrightarrow R \notin R$ is always true in all theories, we conclude that in $N B G$, after assuming $M(R)$, there is Russell's paradox and there is no Russell's paradox. Of course this double-paradox would imply that $\neg M(R)$, but we proved the statement $\neg M(R)$ via the Maximality Principle in a shorter and easier way, avoiding paradoxes and formal logical tricks with an impredicative definition of $R$ under assumption $M(R)$, together with the well-formed or well-defined formula $R \in R$. There is another reason for us to avoid the derived rule of proof by definition. If $M(R)$, then by the Axiom of Power, there exists the set $\mathcal{P}(R)$ of all subsets of $R$, and each element $X \in \mathcal{P}(R)$ satisfies Russell's condition $X \notin X$. By Cantor's theorem, the power of $\mathcal{P}(R)$ is greater than the power of $R$, and thus, by Cantor-Bernstein's theorem, $\mathcal{P}(R)$ is not a subset of $R$. So, the statement $M(R) \Longrightarrow \neg(\mathcal{P}(R) \subseteq R)$ is true. On the other hand, using the derived rule of proof, by definition we conclude that the statement $M(R) \Longrightarrow \mathcal{P}(R) \subseteq R$ is true in spite of the fact that $R \in \mathcal{P}(R)$ and $R \notin R$. We can use the definition of $R$ for every element $X$ of $\mathcal{P}(R)$ except $X=R$ to prove that $X \in R$, because all $X \in \mathcal{P}(R)$ are well-founded, and hence $X \notin X$; but for the well-founded set $R$ we cannot conclude that $R \in R$ because a set $R$ became indefinite. Notice that in $N B G$ Russell's "paradox" coincides with Cantor's "paradox" as well as with Mirimanoff's "paradox," and after well-ordering $R$, it coincides with Burali-Forti's "paradox" (see all of them below).

Proposition 6. For any formal system (axiomatic set theory) $K$ an inner model of which is the class $\mathbf{V}[\mathcal{U}]$ in $N B G$, after the supposition $M(R)$ for $R=\{X \mid M(X) \wedge X \notin X\}$ the following statement (formula) $R \notin R \Longrightarrow$ $R \in R$ is false.

Proof. It is known that for any theorem $\mathcal{A}$ of $K, \operatorname{Rel}_{\mathbf{V}[\mathcal{U}]} \mathcal{A}$ is also a theorem of $K$ (see [41], Chap. 4, p. 282-283) or if $\mathcal{A}$ is a true statement in $K$, then it is true in any model (see Chap. $1, \S$, Theorem 1 in [19]). By Proposition $4, R \notin R \Longleftrightarrow R \in R$ is false in inner model $\mathbf{V}[\mathcal{U}]$ of $K$, where $\mathbf{V}[\mathcal{U}]$ is the universe in $N B G$ of all well-founded sets. Hence it is false in $K$.

Corollary 2. $R \notin R \Longrightarrow R \in R$ is false in $N B G^{-}$.

Proof. The same universe $\mathbf{V}[\mathcal{U}]$ in $N B G$ is an inner model for $N B G^{-}$. Corollary 3. $R \notin R \Longrightarrow R \in R$ is false in $Z F[\mathcal{U}]+($ AFA). 
Proof. Von Neumann's universe $\mathbf{V}[\mathcal{U}]$ is an inner model for $Z F[\mathcal{U}]+$ (AFA).

Corollary 4. $R \notin R \Longrightarrow R \in R$ is false in Cantor's Naïve Set Theory.

Proof. We turn Cantor's Naïve Set Theory into the following naïve formal system by adding to the first order predicate caculus some naïve axioms, judging by his silence on the issue, used without proving:

(I) Axiom of the singleton. If $X$ is a set, then there exists a set $\{X\}$ which contains $X$ and only $X$ as an element.

(II) Axiom of the union of two disjoint sets. If $X$ and $Y$ are two sets which have no common elements, then there exists a set $X \cup Y$ whose elements are the elements of $X$ and of $Y$, taken together.

(III) Axiom of the power set. If $X$ is a set, then there exists a power set $\mathcal{P}(X)$ whose elements are subsets of $X$.

(IV) Axiom of infinity. There exists an infinite set.

Clearly, von Neumann's universe $\mathbf{V}$ is an inner model for such a formal system (naïve set theory) and for each $X \in \mathbf{V}$ the formula $X \notin X$ is always true and the formula $X \in X$ is always false.

Proposition 7. Russell's collection $R$ in all the above set theories is a proper class, not a set.

Proof. Since in all these theories, after assuming $M(R)$, the statement $R \in R$ is false, we conclude that $R \notin R$ and, by the Maximality Principle, we obtain that $R$ is not maximal. Thus, $\vdash M(R)$.

We can even avoid the definition of $R$. Indeed, assuming $M(R)$, we notice that von Neumann's universe $\mathbf{V}$ is a subclass of $R$. Hence $R \backslash(R \backslash$ $\mathbf{V})=\mathbf{V}$ is a set which contradicts Proposition 5 .

Thus, the set $R$ is not inconsistent because of Russell's famous paradox, or antinomy, as has been believed for more than one hundred years [61] but it is inconsistent because of a different argument, i.e., the Maximality Principle.

Conjecture 2. In general, for any reflexive set $X$, there is no nontrivial predicate (well-formed) $\mathcal{B}(x)$ such that $X=\{x \mid \mathcal{B}(x)\}$, in particular, $\mathcal{B}(X)=M(X) \wedge X \notin X$ does not define the reflexive set $R$ under assumption $M(R)$.

We say "in general" because in $N B G^{-}+($AFA $)$a reflexive set $X \in X$ is uniquely determined by elements $X \backslash\{X\}$. We say "non-trivial predicate" because for each reflexive set $X$ there is a rivial predicate (tautology): $X=\{x \mid x \in X\}$. The problem is that for each reflexive set $X$ the nature of its elements in $X \backslash\{X\}$ is different from the nature of its reflexive element $X \in X$. We know only one example in $N B G^{-}+($AFA) of a unique reflexive set $\Omega$ such that $\Omega=\{\Omega\}$.

\subsection{Russell's set is a parametric set.}


Later in [59], [28], [3] and [4] a parametric version of a real Russellian set (not a proper class) was introduced. For any set $a \in \mathbf{V}[\mathcal{U}]^{-}$(well-founded or non-well-founded, it does not matter) the following set

$$
R_{a}=\{b \in a \mid b \notin b\}
$$

always exists as a set because it is an intersection of the proper class $R$ and the set $a$. As has been observed: "There is nothing paradoxical about $R_{a}$. The reasoning that seemed to give rise to paradox only tells us that $R_{a} \notin a$ ". (See [4], p. 60). That means that the assumption $R_{a} \in a$ gives the paradox $R_{a} \in R_{a} \Longleftrightarrow R_{a} \notin R_{a}$. This is the usual Russellian argument. Consider, e.g., the proof, given by Halmos in [28], p. 6, changing only the notation, which we take from [3]. "Can it be that $R_{a} \in a$ ? We proceed to prove that the answer is no. Indeed, if $R_{a} \in a, R_{a} \in R_{a}$ also (unlikely, but not obviously impossible), or else $R_{a} \notin R_{a}$. If $R_{a} \in R_{a}$, then, by (23), the assumption $R_{a} \in a$ yields $R_{a} \notin R_{a}-$ a contradiction. If $R_{a} \notin R_{a}$, then, by (23) again, the assumption $R_{a} \in a$ yields $R_{a} \in R_{a}$ - a contradiction again. This completes the proof that $R_{a} \in a$ is impossible, so that we must have $R_{a} \notin a "$.

In [59] Zermelo showed, by this relative Russell's paradox, that the domain $\mathcal{B}$ of his sets is not a set.

We see that here the implication $R_{a} \notin R_{a} \Longrightarrow R_{a} \in R_{a}$ is false, too, as it was in Russell's argument. If one, still believing in Russell's paradox, uses the derived rule of proof by definition and states that the implication $R_{a} \notin R_{a} \Longrightarrow R_{a} \in R_{a}$ is true, then the result $R_{a} \notin a$ follows logically from the double-paradox $R_{a} \notin R_{a} \Longrightarrow R_{a} \in R_{a}$ is true and $R_{a} \notin R_{a} \Longrightarrow R_{a} \in R_{a}$ is false, since $R_{a} \in R_{a} \Longrightarrow R_{a} \notin R_{a}$ is always true.

Here is a shorter correct proof of this statement based on the Maximality Principle.

Proposition 8. $R_{a} \notin a$.

Proof. It is clear, that $R_{a} \notin R_{a}$ because otherwise, (i.e., $R_{a} \in R_{a}$ ), it would be a member of $a$ and, therefore, by (23), $R_{a} \notin R_{a}$. Thus, we have proved that $R_{a} \notin R_{a}$. Notice the implication that $R_{a} \in R_{a} \Longrightarrow R_{a} \notin R_{a}$ is always true in similar situations.

Suppose now that $R_{a} \in a$. Then $R_{a} \cup\left\{R_{a}\right\}$ is a subset of $a$; moreover, it is a subset of $a$ whose elements do not contain themselves, in particular, $R_{a}$, as we have proved above. Therefore, $R_{a}$ is a proper subset of $R_{a} \cup\left\{R_{a}\right\}$; i.e., $R_{a} \subset R_{a} \cup\left\{R_{a}\right\} \subset a$ which, by (23), is in contradiction with the maximality of $R_{a}$, since it is the set of all elements $b$ of $a$ such that $b \notin b$. Consequently, $R_{a} \notin a$.

Remark 10. In spite of the gap in Halmos's proof, the author of this paper cannot refrain from relating a witty observation made by Paul R. 
Halmos (the author once met him and appreciated his wit as well as his brilliant lectures and books): "The most interesting part of this conclusion is that there exists something (namely $R_{a}$ ) that does not belong to $a$. The set $a$ in this argument was quite arbitrary. We have proved, in other words, that nothing contains everything, or, more spectacularly, there is no universe. 'Universe' here is used in the sense of 'universe of discourse,' meaning, in any particular discussion, a set that contains all the objects that enter into that discussion. In older (pre-axiomatic) approaches to set theory, the existence of a universe was taken for granted, and the argument in the preceding paragraph was known as the Russell paradox. The moral is that it is impossible, especially in mathematics, to get something for nothing. To specify a set, it is not enough to pronounce some magic words (which may form a sentence such as ' $x \notin x$ '); it is necessary also to have at hand a set to whose elements the magic words apply". (Ibid., p. 6-7).

\subsection{Zermelo's paradox.}

Zermelo founded his own paradox independently of Russell and said in 1908 that he had mentioned it to Hilbert and other people already before 1903. Later, in 1936, in his letter to Scholz, he wrote that the set-theoretical paradoxes were often discussed in the Hilbert circle around 1900, and he himself had at that time given a precise formulation of the paradox which was later named after Russell (see [63]). We shall see below that Zermelo's opinion was mistaken, and that Russell's and his paradoxes are similar but not the same. Zermelo's paradox is the following: a set $M$ that comprises as elements all of its subsets is inconsistent. Indeed, consider the set $M_{0}$ of all elements of $M$ which are not elements of themselves (e.g., the empty set is in $M_{0}$ ) This set is a subset of $M$ and hence by assumption on $M, M_{0} \in M$. If $M_{0} \in M_{0}$, then $M_{0}$ is not a member of itself. Hence $M_{0} \notin M_{0}$ and since $M_{0} \in M, M_{0} \in M_{0}$ : contradiction. (See [64], § 2.4; [61], p. 507). We notice the same mistake: the implication $\left(M_{0} \notin M_{0} \wedge M_{0} \in M\right) \Longrightarrow M_{0} \in M_{0}$ is false.

We can repeat said above, if one still believing in Russell's paradox uses the derived rule of proof by definition and states that the implication $\left(M_{0} \notin M_{0} \wedge M_{0} \in M\right) \Longrightarrow M_{0} \in M_{0}$ is true, then the result $M_{0} \notin M$ follows logically from the double-paradox $\left(M_{0} \notin M_{0} \wedge M_{0} \in M\right) \Longrightarrow M_{0} \in M_{0}$ is true and $\left(M_{0} \notin M_{0} \wedge M_{0} \in M\right) \Longrightarrow M_{0} \in M_{0}$ is false.

Here is the solution of Zermelo's paradox based on the Maximaliti Principle.

Proposition 9. There is no set $M$ such that it comprises as elements all of its subsets.

Proof. Suppose the contrary, and such set $M$ exists. Then $M_{0}=\{X \in$ $M \mid X \notin X\}$ is well-defined and $M_{0} \in M$. If $M_{0} \in M_{0}$ in any possible way, 
then $M_{0} \in M_{0} \in M_{0}$ and, clearly, by definition of it, $M_{0} \notin M_{0}$. (The first part of Zermelo's proof is correct.) By axiom (I), the singleton $\left\{M_{0}\right\}$ also exists and it is a subset of $M$, i.e., $\left\{M_{0}\right\} \subset M$ (because $M_{0} \subset M$ and, by definition of $\left.M, M_{0} \in M\right)$. By axiom (II), we obtain that $M_{0} \cup\left\{M_{0}\right\}$ is also a subset of $M$ and consists of elements which are not elements of themselves because in addition to all elements of $M_{0}$ with such a condition, $M_{0}$ itself satisfied the same condition, i.e., $M_{0} \notin M_{0}$, as was proved in the first part. Clearly, $M_{0}$ is a proper subset of $M_{0} \cup\left\{M_{0}\right\}$; i.e., $M_{0} \subset M_{0} \cup\left\{M_{0}\right\}$ which is in contradiction with the maximality of $M_{0}$. Consequently, $M$ doest not exist.

Notice also that contrary to Russell's "set" $R$, which is not a set but does exist as a proper class, Zermelo's set $M$ does not exist as a proper class at all; in other words, there is no mathematical object such as Zermelo's set $M$, but there is an object $R$ in $N B G^{-}$and in $N B G$. Moreover, in the latter it is called the Universe $\mathbf{V}[\mathcal{U}]$ or the class of all well-founded sets WF. That is why Zermelo's and Russell's paradoxes are meaningfully different. $M$ cannot be a proper class because in its rigid definition $M$ has to be an element of $M$, which is impossible for proper classes. If of course one distinguishes sets and proper classes, then $M$ is an object of set theory (a proper class) such that it comprises as elements all of its subsets (not proper subclasses) but $M$ in this case is not defined in the original sense of Zermelo.

\section{Other set-theoretical paradoxes}

The same argument is valid for the "set" On (Burali-Forti's "paradox" [13]), for the "set" Card of all cardinal numbers (Cantor's "paradox," first mentioned in the letter to Dedekind of August 31. 1899, and later in [17]), for the "set" $\mathcal{U}$ (Hilbert's "paradox" [61], p. 505), and for the "set" WF (Mirimanoff's "paradox" [43]).

We shall also prove that the family NWF of all non-well-founded sets is not a set but a proper class and it does not concern Russell-like paradoxes at all. As above all proofs will be based on Maximality Principle. Admittedly, it was implicitly used by Cantor in proving that the second number-class set is not countable [16], $\S 16$, [Theorem] D. His proof takes up at least one page-more precisely, 32 lines). Now this proof requires only five lines.

Proposition 10. For each initial ordinal $\omega_{\alpha}$, the set of all ordinals $\lambda$ such that $\lambda<\omega_{\alpha}$ cannot be of a power smaller than the power of $\omega_{\alpha}$.

Proof. Indeed, if the power of the set $X=\left\{\lambda \mid \lambda<\omega_{\alpha}\right\}$ were smaller than the power of $\omega_{\alpha}$, then the power of the next ordinal number $\beta=$ $X \cup\{X\}$ would be smaller than the power of $\omega_{\alpha}$, too. Since $\beta$ is greater than each ordinal $\lambda$ in $X$, it is not a member of $X$. Hence $X \subset X \cup\{\beta\}$; 
i.e., $X$ is a proper subset of $X \cup\{\beta\}$, which is in contradiction with the maximality of $X$.

\subsection{Burali-Forti's paradox, or the antinomy of the greatest or- dinal.}

The earliest antinomy in set theory was published in 1897 in [13]. It has, however, a non-trivial history, starting out from the fact that there was nothing paradoxical in it, since in [13] there was no contradiction. This is because Burali-Forti had misconstrued Cantor's definition of a well-odered set, and used his own notion of a different kind of ordered sets which he called "perfectly ordered classes", and proved that such classes are non-wellordered. Russell [54], p. 323, reformulated the argument of Burali-Forti as a contradiction and gave it its present name. (This observation was borrowed from [24], p. 306-307, and [61], p. 350). And we will finish this story with an amusing remark given by Halmos: "The contradiction, based on the assumption that there is a set of all ordinals, is called the BuraliForti paradox. (Burali-Forti was one man, not two.)" (See [28], p. 80.) We are going to show that Russell and others did not supply Burali-Forti's paradox with a contradiction, although there are axiomatic systems where this contradiction arises.

Look at the modern explanation of Russell's correction of Burali-Forti's antinomy of the greatest ordinal, e.g., in [61], p. 350. "Let $\Omega$ be the ordinal number of the well-ordered set of all ordinals, No. But, for every ordinal $\alpha, \alpha+1>\alpha$. Thus $\Omega+1>\Omega$. But, for every $\alpha \in$ No, $\alpha \leq \Omega$. Thus $\Omega+1 \leq \Omega$.

Recall that, due to Cantor, "ordinal numbers" are ordinal types of "wellordered aggregates", i.e., well-ordered sets. Proper classes of equivalent well-ordered sets can be represented by von Numann's method [49], by choosing a canonical set-representative of each proper class, that is to define an ordinal as a set $\alpha$ of sets $X$ which is well-ordered by the relation $\epsilon$ between its elements and transivity, i.e., if $Z \in Y \in X$, then $Z \in X$, starting, e.g., with the empty set, i.e., $\emptyset ;\{\emptyset,\{\emptyset\}\} ;\{\emptyset,\{\emptyset\},\{\emptyset,\{\emptyset\}\}\} ; \ldots$. (It would perhaps have been fairer to say that the idea of this method for the first time was given by Mirimanoff [43], p. 46.) The collection of all ordinals On is well ordered by the relation $\in$ (see [18], p. 8) and class transitive; i.e., if $Z \in Y \in \mathbf{O n}$, then $Z \in \mathbf{O n}$. Note that the class of all ordinals No above is in bijection with On; moreover, they are order isomorphic. Note also that in $N B G$ all ordinals are well-founded sets. Thus, for each $X \in \alpha$ we have $X \notin X$, in particular, $\alpha \notin \alpha$, and moreover, we can even omit "well" in "well-ordered" (see, e.g., [41], Chap. 4, §5, Exercise 4.84). The 
situation in $N B G^{-}$and in Naïve Set Theory is more complicated: "wellordered" is sufficient and $\alpha \in \alpha$ can happen. Nevertheless, we remember that in general the relations $\alpha \in \alpha$ as well as $\alpha \in \beta \in \alpha$ are indeterminate.

Proposition 11. On is a proper class, not a set.

Proof. Let On be a collection of all transitive, well-founded sets ordered by the relation $\in$, i.e., we are in $N B G$. Suppose that $\mathbf{O n}$ is a set. Thus, it is a transitive set well-ordered by the relation $\in$ and hence is an ordinal. Then, by $(\mathbf{I}),\{\mathbf{O n}\}$ is also a set, and, by $(\mathbf{I I}), \mathbf{O n} \cup\{\mathbf{O n}\}$ is a set which is evidently well-ordered by the relation $\in$ and transitive, too. Consequently, $\mathbf{O n} \cup\{\mathbf{O n}\}$ is an ordinal. (Notice that in $N B G$ On $\notin \mathbf{O n}$ and $\{\mathbf{O n} \cup$ $\{\mathbf{O n}\}\} \notin \mathbf{O n}$ because all sets in $N B G$ are well-founded.) Moreover, by the same argument, $\{\mathbf{O n} \cup\{\mathbf{O n}\}\}$ and $\mathbf{O n} \cup\{\mathbf{O n} \cup\{\mathbf{O n}\}\}$ are sets and thus On is a proper subset of $\mathbf{O n} \cup\{\mathbf{O n} \cup\{\mathbf{O n}\}\}$ because of the relation noted above; i.e., On $\cup\{\mathbf{O n}\} \notin \mathbf{O n}$. The relation On $\subset \mathbf{O n} \cup\{\mathbf{O n} \cup\{\mathbf{O n}\}\}$ is in contradiction with the maximality of On. Thus On is not a set. It is a proper class since its existence is guaranteed by the predicate: to be an ordinal. If On is a family of all ordinal numbers represented in some other way, then there is an isomorphism $\varphi: \mathbf{O n} \rightarrow \mathbf{O n}$. Thus, the relations On $\in \mathbf{O n}$ and $\mathbf{O n}+1 \in \mathbf{O n}$ are impossible because by isomorphism $\varphi$ applied to these relation the corresponding relations are false in $\mathbf{O n}$.

Remark 11. Burali-Forti's antinomy of the greatest ordinal is seen now as two inconsistent inequalities $\Omega+1>\Omega$ and $\Omega+1 \leq \Omega$. Consequently, we have the relations $\Omega<\Omega+1 \leq \Omega$ in No and hence, by the isomorphism between No and On mentioned above, we obtain the relations $\Omega \in \Omega \cup$ $\{\Omega\} \in \Omega$ in $\mathbf{O n}$, which are indeterminate, as we have already seen many times. So in the classical argument, to conclude by definition that $\Omega+1 \in \Omega$ is false. (The true state of affairs is that after the supposition that $\mathbf{O n}=\Omega$ is a set, the definition of $\mathbf{O n}$ has been radically changed; i.e., each possible relation On $\in \mathbf{O n}$ or $\Omega \in \Omega \cup\{\Omega\} \in \Omega$ changes the set On itself, and that is why On is indeterminate.) Consequently, when Russell supposedly showed a contradiction in the Burali-Forti example, he was wrong. If we suppose that $\mathbf{O n}$ is a set, then naturally $\mathbf{O n} \cup\{\mathbf{O n}\}=\Omega+1$ is an ordinal and $\Omega+1>\Omega=$ On. But $\Omega \cup\{\Omega\} \notin \Omega$, i.e., $\Omega+1 \not \leq \Omega$. Because $\Omega=\mathbf{O n}$ is a well-founded set in $N B G$, and in $N B G^{-}$as well as in Naïve Set Theory, the relations $\Omega \cup\{\Omega\} \in \Omega$ are indeterminate. Moreover, by the isomorphism of classes of all ordinals represented in any way, the above relations are false in On, i.e., in the collection of all well-founded, transitive sets ordered by the relation $\in$. In our proof of the inconsistency of the set On we omit this erroneous step $\mathbf{O n} \cup\{\mathbf{O n}\} \in \mathbf{O n}$ but add a new ordinal $\mathbf{O n} \cup\{\mathbf{O n}\}$ (different from each element of $\mathbf{O n}$ ) to $\mathbf{O n}$ and obtain a larger set than $\mathbf{O n}$, and this is a real contradiction, because $\mathbf{O n}$ is a collection of all ordinals. 


\subsection{Cantor's paradox.}

The historically second set-theory antinomy belongs to Cantor: the set $\mathbf{V}^{*}$ of all sets (which was called later "Cantor's paradise") is inconsistent or paradoxical because the power set $\mathbf{P} \mathbf{V}^{*}$ is always of a greater cardinality than $\mathbf{V}^{*}$, but at the same time $\mathbf{P} \mathbf{V}^{*} \subseteq \mathbf{V}^{*}$ since $\mathbf{V}^{*}$ is the most inclusive set of sets. Notice that the same mistake obtains: the relation $\mathbf{P V}^{*} \subseteq \mathbf{V}^{*}$ implies, in particular, $\mathbf{V}^{*} \in \mathbf{V}^{*}$ since $\mathbf{V}^{*} \in \mathbf{P V}^{*}$. But the relation $\mathbf{V}^{*} \in$ $\mathbf{V}^{*}$ is many-valued and indeterminate, as we saw above, and therefore, false. Thus there is no real paradox or contradiction in Cantor's argument; moreover, in this case Cantor's paradox is not a paradox at all. But what was correctly asserted via Cantor's "paradox" was the following:

Proposition 12. $\mathbf{V}^{*}$ is a proper class, not a set, or in Cantor's denomination $\mathbf{V}^{*}$ is an "inconsistent system".

Proof. Suppose that $\mathbf{V}^{*}$ is a set. Then, by the Power-set Axiom, the collection (system) $\mathbf{P} \mathbf{V}^{*}$ of all subsets of $\mathbf{V}^{*}$ is a set. It is clear, by $(\mathbf{I})$, that there is an injection $i: \mathbf{V}^{*} \rightarrow \mathbf{P V}^{*}$, given by $i(X)=\{X\}$. On the other hand, $\mathbf{P V}^{*}$ is not a subset of $\mathbf{V}^{*}$. Otherwise, there would be an embedding $j: \mathbf{P} \mathbf{V}^{*} \rightarrow \mathbf{V}^{*}$ and, by the Cantor-Bernstein theorem, $\mathbf{P} \mathbf{V}^{*}$ and $\mathbf{V}^{*}$ would be equivalent, which is false because of Cantor's theorem $\left|\mathbf{P V}^{*}\right|>\left|\mathbf{V}^{*}\right|$. Thus, there exists an element $X \in \mathbf{P V}^{*}$ such that $X$ is a set and $X \notin \mathbf{V}^{*}$. Then, by $(\mathbf{I}),\{X\}$ is a set and, by $(\mathbf{I I}), \mathbf{V}^{*} \cup\{X\}$ is a set. Moreover, $\mathbf{V}^{*} \subset \mathbf{V}^{*} \cup\{X\}$, i.e., $\mathbf{V}^{*}$ is a proper subset of $\mathbf{V}^{*} \cup\{X\}$, which is in contradiction with the maximality of $\mathbf{V}^{*}$. Thus $\mathbf{V}^{*}$ is not a set but a proper class, given by the predicate $X=X$, i.e., $\mathbf{V}^{*} \stackrel{\text { def }}{=}\{X \mid X=X \& \exists\{X\}\}$ and called the universal class (see [27], p. 124). (Of course, we could take $X=\mathbf{V}^{*}$ at the beginning, and since $\mathbf{V}^{*} \in \mathbf{V}^{*}$ is indeterminate, conclude that $\mathbf{V}^{*} \notin \mathbf{V}^{*}$ and, by supposition that $\mathbf{V}^{*}$ is a set, obtain, by (I) and (II), that $\mathbf{V}^{*}$ is a proper subset of $\mathbf{V}^{*} \cup\left\{\mathbf{V}^{*}\right\}$. In other words, we could repeat our method of maximality; but we wished to find a mistake in Cantor's argument.)

Cantor himself reached the faulty conclusion that $\mathbf{P} \mathbf{V}^{*} \subseteq \mathbf{V}^{*}$, which is in contradiction with their cardinality, and obtained a supposed "paradox". But it was only a presumption, which was proved to be false by Cantor's theorem $\left|\mathbf{P V}^{*}\right|>\left|\mathbf{V}^{*}\right|$, nothing more. Therefore, there is no Cantor's paradox. It would be a paradox if Cantor could prove that $\mathbf{P V}^{*} \subseteq \mathbf{V}^{*}$. Meanwhile his conclusion $\mathbf{P V}^{*} \subseteq \mathbf{V}^{*}$ was made via the Definition of $\mathbf{V}^{*}$; but after the assumption that $\mathbf{V}^{*}$ was a set, the Definition of $\mathbf{V}^{*}$ was radically changed, and $\mathbf{V}^{*} \in \mathbf{V}^{*}$ became automatically many-valued. In other words, Cantor had to prove that each set $X \in \mathbf{P V}^{*}$ is an element of $\mathbf{V}^{*}$. He could do it by Definition of $\mathbf{V}^{*}$ except in the one case when 
$X=\mathbf{V}^{*} \in \mathbf{P} \mathbf{V}^{*}$, because in $\mathbf{V}^{*}$ there are many (actually all) reflexive sets $X$ including all skands $X=X_{(0, \alpha)}, \alpha \geq \omega$, such that $X_{(0, \alpha)}=X_{(1, \alpha)}$. Then, since $\mathbf{V}^{*} \in \mathbf{V}^{*}$ as Cantor concluded by Definition, a reflexive set $\mathbf{V}^{*}$ should be at once the skands of all lengths $\alpha \geq \omega$, which is impossible. Thus, it is impossible to prove that $\mathbf{P V}^{*} \subseteq \mathbf{V}^{*}$.

Absolutely by a similar argument Cantor proved that the collection Card of all cardinal numbers is not a set (August 31, 1899, letter to Dedekind) and Cantor made the following conclusion: “... the system Card is not a set. That is why there exist certain pluralities which are not at the same time wholes (unities), i.e., pluralities for which the real 'mutual being of all their elements' is impossible. They are those I call the 'inconsistent systems'; as for the rest I call them the 'sets' " [62].

Here is a correct proof of Cantor's statement.

Proposition 13. Card is a proper class, not a set, or in Cantor's denomination Card is an "inconsistent system".

Proof. Suppose that Card is a set. It is known that Card is in a bijection with the family In of all finite numbers together with the all initial ordinals $\omega_{\kappa}$ of On which we identify with the initial segments $\left(0, \omega_{\kappa}\right)$ of $\mathbf{O n}$, $\kappa \in \mathbf{O n}$ (if $\kappa=0$, then the corresponding segment is the empty set). Since we supposed that Card is a set, therefore In is a set, by the Replacement Axiom for sets, and we obtain that the discrete sum $X=\coprod_{\omega_{\kappa} \in \mathbf{I n}}\left[0, \omega_{\kappa}\right)$ is also a set, by the Union Axiom for sets, and, by the Power-set Axiom, $\mathbf{P} X$ is a set. Hence there exists an initial ordinal $\omega_{\lambda}$ such that $\mathbf{P} X$ and $\left[0, \omega_{\lambda}\right)$ are bijective. It is clear that $\omega_{\lambda}>\omega_{\kappa}$, for each $\omega_{\kappa} \in \mathbf{I n}$ (because of Cantor's theorem: $\left.\left|\mathbf{P}\left[0, \omega_{\kappa}\right)\right|>\left|\left[0, \omega_{\kappa}\right)\right|, \kappa \in \mathbf{O n}\right)$, which is in contradiction with the maximality of In. Thus Card is not a set but a proper class, since Card $\subset \mathbf{V}^{*}$.

Remark 12. Notice that from the fact that On is not a set but a proper class, as has been proved above, it does not follow that its subclass Card is also a proper class. On the other hand, there is a bijection between $\mathbf{O n}$ and Card because there is an injection $j:$ On $\rightarrow$ Card, given by $j(\kappa)=\omega_{\kappa}$, $\kappa \in \mathbf{O n}$, and we apply the Cantor-Bernstein theorem. Thus Card is not a set, but a proper class.

\subsection{Hilbert's paradox.}

At least earlier than 1905 Hilbert formulated a paradox of his own which he had considered "purely mathematical" in the sense that it did not make use of notions from Cantor's theory of cardinals and ordinals. Hilbert never published the paradox: "I have never published this contradiction, but it is known to set theorists, especially to G. Cantor" [33], p. 204. 
Let us quote it from [61], p. 505-506. "The paradox is based on a special notion of set which Hilbert introduces by means of two set formation principles starting from the natural numbers. The first principle is the addition principle (Additionsprinzip). In analogy to the finite case, Hilbert argued that the principle can be used for uniting two sets together 'into a new conceptual unit', a new set that contains the elements of both sets. This operation can be extended: 'In the same way, we are able to unite several sets and even an infinitely many into a union.' The second principle is called the mapping principle (Belegungsprinzip). Given a set $\mathcal{M}$, he introduces the set $\mathcal{M}^{\mathcal{M}}$ of self-mappings (Selbstbelegungen) of $\mathcal{M}$ to itself. A self-mapping is just a total function ('transformation') which maps the elements of $\mathcal{M}$ to elements of $\mathcal{M}$.

Now, he considers all sets which result from the natural numbers 'by applying the operations of addition and self-mapping an arbitrary number times.' By the addition priciple, which allows us to form the union of arbitrary sets, one can 'unite them all into a sum set $\mathcal{U}$, which is welldefined.' In the next step the mapping principle is applied to $\mathcal{U}$, and we get $\mathcal{F}=\mathcal{U}^{\mathcal{U}}$ as the set of all self-mappings of $\mathcal{U}$. Since $\mathcal{F}$ was built from the natural numbers, by using the two principles alone, Hilbert concludes that it has to be contained in $\mathcal{U}$. From this fact he derives a contradiction.

Since there are 'not more' elements in $\mathcal{F}$ than in $\mathcal{U}$ there is an assignment of the elements $u_{i}$ of $\mathcal{U}$ to elements $f_{i}$ of $\mathcal{F}$ such that all elements of $f_{i}$ are used. Now one can define a self-mapping $g$ of $\mathcal{U}$ which differs from all $f_{i}$. Thus, $g$ is not contained in $\mathcal{F}$. Since $\mathcal{F}$ contains all self-mappings, we have a contradiction. In order to define $g$, Hilbert used Cantor's diagonalization method. If $f_{i}$ is a mapping $u_{i}$ to $f_{i}\left(u_{i}\right)=u_{f_{i}^{i}}$ he chooses an element $u_{g^{i}}$ different from $u_{f_{i}^{i}}$ as the image of $u_{i}$ under $g$. Thus, we have $g\left(u_{i}\right)=u_{g^{i}} \neq$ $u_{f_{i}^{i}}$ and $g$ 'is distinct from any mapping $f_{k}$ of $\mathcal{F}$ in at least one asssignment.' Hilbert finishes his argument with the following obsorvation:

"We could also formulate this contradiction so that, according to the last consideration, the set $\mathcal{U}^{\mathcal{U}}$ is always bigger [of greater cardinality] than $\mathcal{F}=\mathcal{U}$, but according to the former, is an element of $\mathcal{F}=\mathcal{U}$."

Let us comment on the above proof. In spite of the difference between Cantor's and Hilbert's definitions of set (e.g., the empty set set is not in $\mathcal{U}$, and other non-intersections) their proofs and arguments are similar and contain the same mistake. Let us see it in Hilbert's paradox. Hilbert has proved, using Cantor's diagonalization method, only that his supposition that $\mathcal{F} \subseteq \mathcal{U}$ is false, nothing more. Thus, there is no contradiction in such an unfortunate supposition. If he had proved that $\mathcal{F} \subseteq \mathcal{U}$ was true, there would be a real paradox to be named after him. What he has essentially 
proved is that there is an element $g$ of $\mathcal{F}$ such that it was not an element of $\mathcal{U}$. Then by his first principle $\mathcal{U} \cup \mathcal{F}$ is a set; moreover, $\mathcal{U}$ is a proper subset of $\mathcal{U} \cup \mathcal{F}$ because $b$ is not in $\mathcal{U}$ and it is in $\mathcal{U} \cup \mathcal{F}$. Thus, by the universality of $\mathcal{U}$, (i.e., $\mathcal{U}$ is the set of all Hilbert's sets), $\mathcal{U}$ is not a Hilbert

set, nor is $\mathcal{F}=\mathcal{U}^{\mathcal{U}}$ a Hilbert set. There is no paradox here at all. There is only a proof by contradiction, and the supposition that $\mathcal{U}$ is a set is simply false.

6.4 Mirimanoff's paradox.

In [43], p. 43, Mirimanoff was actually concerned with the collection WF of all well-founded sets, and presented his paradox:

$$
\mathbf{W F} \in \mathbf{W F} \Longleftrightarrow \mathbf{W F} \notin \mathbf{W F}
$$

as an analogue of Russell's paradox. And he concluded that the set WF did not exist, i.e., in Cantor's terminology, it is an inconsistent system, or in modern terminology, it is not a set. His argument is the following. It is clear that $\mathbf{W F} \notin \mathbf{W F}$ because if it were not so and $\mathbf{W F} \in \mathbf{W F}$, then there would exist an infinite descending chain in $\mathbf{W F}$, e.g., $\mathbf{W F} \ni \mathbf{W F} \ni \mathbf{W F} \ni \ldots$ and we would obtain that $\mathbf{W F}$ is non-well-founded and hence $\mathbf{W F} \notin \mathbf{W F}$.

On the other hand, if $\mathbf{W F}$ is non-well-founded; there exists an infinite chain $x_{0} \ni x_{1} \ni x_{2} \ldots$ in $\mathbf{W F}$ for some $x_{0} \in \mathbf{W F}$, and then $x_{0}$ is evidently non-well-founded, which is wrong. Therefore, $\mathbf{W F}$ is well-founded. Hence $\mathbf{W F} \in \mathbf{W F}$; contradiction.

The last conclusion is false because $\mathbf{W F} \in \mathbf{W F}$ is indeterminate as we saw above more than once.

Here is a correct proof of the following

Proposition 14. WF is a proper class, not a set.

Proof. Assume that $\mathbf{W F}$ is a set. Then by $(\mathbf{I})$ the singleton $\{\mathbf{W F}\}$ is also a set. Since all elements of $\mathbf{W F}$ are well-founded we conclude that $\mathbf{W F}$ is not an element of $\mathbf{W F}$ because if it were, then there would be an evident infinite descending $\in$-sequence $\mathbf{W F} \ni \mathbf{W F} \ni \mathbf{W F} \ni \ldots$ Thus $\mathbf{W F}$ and $\{\mathbf{W F}\}$ are two sets without common elements. Then, by (II), $X^{\prime}=\mathbf{W F} \cup\{\mathbf{W F}\}$ is also a set. Moreover, its elements are well-founded and $\mathbf{W F}$ is a proper subset of $X^{\prime}$ because $\mathbf{W F}$ is a member of $X^{\prime}$ but not a member of WF. This is in contradiction with the maximality of WF because it is the well-founded universe, since $\mathbf{W F}$ is the collection of all well-founded sets. Consequently, the assertion that WF is a set is false. By the well-founded predicate and the Existence Axiom for a class, WF is a proper class.

In $N B G^{(1)}$ we have the following collection:

$$
\mathbf{T}=\{X \mid X \in X\}
$$


which is symmetric to Russell's "set" $R=\{X \mid X \notin X\}$ [21], p. 277, and on the assumption that it is a set it does not look paradoxical or inconsistent. We are not saying that $\mathbf{T} \in \mathbf{T}$, because otherwise, the relation $\mathbf{T} \in \mathbf{T}$ cannot even be called indeterminate as above; it is meaningless as Russell tells us in [58], p. 80. Indeed, $\mathbf{T}$ contains, e.g., all skands of arbitrary lengths; $\mathbf{T} \in \mathbf{T}$, if it were the case, would be a skand of a fixed length, and other skands of different lengths would be different from this fixed one. In other words, $\mathbf{T}$ cannot be a skand of all lengths at once. Nevertheless,

Proposition 15. $\mathbf{T}$ is a proper class, not a set.

Proof. Consider for an arbitrary well-founded set $X$, i.e., $X \in \mathbf{W F}$, a self-similar skand $X_{(0, \omega)}$ with the components $X_{i}=X, 0 \leq i<\omega$, and denote by $\mathbf{L}$ the subclass $\left\{X_{(0, \omega)} \mid X \in \mathbf{W F}, X_{i}=X, 0 \leq i<\omega\right\}$ which consists of all such self-similar skands $X_{(0, \omega)}$ with $X_{i}=X, 0 \leq i<\omega$. Clearly, $\mathbf{L} \subset \mathbf{T}$. Since $\mathbf{W F}$ is a proper class then $\mathbf{L}$, which is bijective to it, is also a proper class. Consequently, $\mathbf{T}$ is also a proper class because on the contrary, if $\mathbf{T}$ were a set the relation $\mathbf{L} \subset \mathbf{T}$ would imply that $\mathbf{L}$ were a set.

All these results are well-known. What is new is the simple proofs of them and the unexpected discovery that the classical set-theoretical paradoxes are not paradoxes at all. Moreover, we maintain that the substance of all such "paradoxical sets", actually proper classes, is in the following simplest lemma of such a kind of proposition.

Lemma 1. The collection $\mathbf{S}$ of all singletons in $\mathbf{W F}$ is a proper class, not a set.

Proof. Suppose the contrary, and $\mathbf{S}$ is a set. By $(\mathbf{I}),\{\mathbf{S}\}$ and $\{\{\mathbf{S}\}\}$ are also well-founded sets. Moreover, $\{\mathbf{S}\} \notin \mathbf{S}$ because all elements of $\mathbf{S}$ are well-founded. Then, by (II), the disjoint union $\mathbf{S} \cup\{\{\mathbf{S}\}\}$ is also a set and $\mathbf{S}$ is a proper subset of $\mathbf{S} \cup\{\{\mathbf{S}\}\}$ which contradicts the maximality of S.

Corollary 5. That propositions 4, 6-12 together with Generalized Lemma 1, i.e., the collection of all singletons in $\mathbf{V}[\mathcal{U}]^{-}$form a proper class, not a set, is a consequence of Lemma 1.

The Proof is evident. One can easily find in all these cases that $\mathbf{S}$ is a subclass of the supposed "sets" or that there is a subclass of the supposed "sets" which is in bijection with $\mathbf{S}$. Then the assumption that the wider class is a set implies, by an axiom in $N B G$ which says that the intersection of any set $X$ with an abitrary class $Y$ is a set (see, [41], Chap. 4, Axiom S), that $\mathbf{S}$ is a set, contrary to Lemma 1 . Indeed, the simplest proof is of the Generalized Lemma 1, Proposition 12, Proposition 14, because evidently the class of all well-founded singletons is a subclass of all singletons as well as $\mathbf{S} \subset \mathbf{V}^{-}$and $\mathbf{S} \subset \mathbf{W F}$, and the statements made of them hold. 
Since, by von Neumann's axiom $\mathbf{N}, \mathbf{V}^{-} \approx$ On there is a subclass of $\mathbf{N}, \mathbf{O n}$ which is equivalent to $\mathbf{S}$, and by the injection $j: \mathbf{O n} \rightarrow$ Card (Remark 12), the statements of Propositions 11 and 12 hold. As to Russell's set $R$ (Proposition 5), it contains $\mathbf{S}$ as a subclass because, for each well-founded set $X \in R$, the singleton $\{X\}$ is also well-founded and $\{X\} \in R$; otherwise, $\{X\} \in\{X\}$ and hence $\{X\}=X$ and the latter is a non-well-founded set. In $\mathbf{T}$ there is a subclass of self-similar skands $X_{(0, \omega)}$ whose components $X_{i}=X, 0 \leq n<\omega, X \in \mathbf{S}$, and thus Proposition 15 holds.

Remark 13. In [4] there is another, also very short proof of a generalized Lemma 1, but "from the top to the bottom", whereas in our proof we are going "from the bottom to the top". Here it is. If $\mathbf{S}$ in $\mathbf{V}[\mathcal{U}]^{-}$were a set, its union would be a set. But $\bigcup \mathbf{S}$ contains the proper class $\mathbf{V}[\mathcal{U}]^{-}$of all sets. This is because for all sets $a,\{a\} \in \mathbf{S}$. Since $\bigcup \mathbf{S}$ is both a proper class and a set, we have a contradiction. (See [4], p. 338, and note that in [4] it has already been proved that the class of all sets is a proper class, although via Russell's paradox; see Ibidem, p. 16).

\section{Comparison of $N B G^{(1)}$ with some other approaches to non- well-founded sets}

First of all, notice that objects $X^{(1)}$ in $\mathbf{V}[\mathcal{U}]^{(1)}$ are essential extensions of some but not all extraordinary sets in the sense of Mirimanoff [43], [44], [45] as well as non-well-founded sets in [22]. In [45], p. 33, Mirimanoff considered extraordinary sets in the following form:

$$
E=\{y, z, \ldots a, b, c, \ldots\}
$$

where sets $y, z, \ldots$ depend themselves on $E$; in particular, he considered sets in the form

$$
E=\{E, a, b, c, \ldots\} .
$$

In $N B G^{(1)}$ we consider not only the latter form and those which are self-similar skands, or circular skands of period 1, but also skands or extraordinary sets of the form

$$
E=\{a, b, c, \ldots,\{E, x, y, z, \ldots\}\},
$$

i.e., circular skands of period 2, and of course we consider also circular skands of arbitrary finite period $n$. But we do not consider extraordinary sets, or self-similar skands, e.g., of the form

$$
E=\{E, a, b, c, \ldots,\{E\}\}
$$

which is a particular case of (26). This restriction of ours is done knowingly and it is similar to the restriction of well-founded sets in comparison with 
non-well-founded sets which can be called 0-rank self-similar skands (wellfounded sets), 1-rank self-similar skands (circular sets of period 1), and we could postulate the existence of self-similar skands of arbitrary finite rank. For our purposes we need such a restriction. So, skands are not extensions of all of Mirimanoff's extraordinary sets of the form (26).

On the other hand, the definition (26) provides the possibility of the existence of skands whose lengths are greater than $\omega$. However nothing was said in [45] about such things, and it appears that Mirimanoff tacitly supposed that the length of skands was equal to $\omega$. Only a single footnote on page 12 in [22] says that " $\in_{\mu}$-constituent of relation (i.e., $\in$-chains of length $\mu$ ) can be defined for an arbitrary ordinal number $\mu$, but we consider in this paper only finite numbers $\mu$ ". Nevertheless, this footnote, given by Elkund, does not determine the fact that non-well-founded objects, presented in [22], are skands of $\omega$-length in our terminology, and hence non-well-defined. So, skands in $N B G^{(1)}$ are essential extensions of extraordinary sets of length $\omega$.

Now we want to compare $N B G^{(1)}$ with Aczel's model or theory of nonwell-founded sets [1] which was motivated by Robin Milner's work [42] on computer science modeling of concurent processes. Aczel's model of so-called hypersets was successfully applied to the treatment of the Liar paradox [3] and various other vicious circle phenomena [4].

Starting out with the Zermelo-Frankel set axiomatic system ZFC, which includes the axiom of choice and the axiom of foundation, Aczel rejected the latter and proposed his own Anti-Foundation Axiom (AFA for short); and with a natural correction of the Axiom of Extensionality he obtained the set axiomatic system $\mathbf{Z F A}^{-}+\mathbf{A F A}$, which is consistent on the assumption that $\mathbf{Z F C}$ is consistent.

The binary relation $x \in y$ Aczel pictures as an element of a graph $x \longrightarrow y$ with nodes $x, y$ and edge $(x, y)$ between them; finite $x_{0} \longrightarrow x_{1} \longrightarrow \ldots \longrightarrow$ $x_{n-1} \longrightarrow x_{n}$ and infinite sequences finite $x_{0} \longrightarrow x_{1} \longrightarrow x_{2} \longrightarrow \ldots$ as well as pointed and accesible graphs, labelled graphs, children, decorations of graphs, etc., are understood in the usual way. So, every set with an $\in$-relation can be pictured by labelled graphs.

Then the Labelled Anti-Foundation Axiom says: Every labelled graph has a unique labelled decoration.

This axiom is a natural extension of Mostowski's Collapsing Lemma that tells us that every well-founded labeled graph has a unique decoration in WF. On the other hand, AFA is a strong restriction of $\mathbf{V}[\mathcal{U}]^{-}$because of Scott's axiom: For any extensional relation $R$ on $A$ and for any not $R$-well-founded $x \in A$ there is no set containing all possible images of $x$ 
under isomorphisms between $(A, R)$ and a transitive structure $(T, \in)$. (Unpublished paper of 1960). As a consequence of this axiom the uniqueness of a "Mostowski collapse" cannot be consistently postulated for non-wellfounded sets. (See details in [26].)

There is an equivalent formulation of AFA which is nearer to our paper called the Solution Lemma.

The Anti-Foundation Axiom: Every flat system of equations has a unique solution, [4], p. 72.

It is enough for us because a particular example of a flat equation is (13). Thus, axiomatic systems $\mathbf{Z F A}{ }^{-}+\mathbf{A F A}$ and $N B G^{(1)}$ are incompatible, although they have mutual objects such as the empty skand $\mathbf{e}_{(0, \omega)}=\{\{\ldots\}\}$ in our paper and $\boldsymbol{\Omega}=\{\{\ldots\}\}$ in [1]. Moreover, the hyperset $\Omega^{*}=\left\{\boldsymbol{\Omega}, \boldsymbol{\Omega}^{*}\right\}$ is equal to $\boldsymbol{\Omega}$ because $\boldsymbol{\Omega}=\{\boldsymbol{\Omega}\}=\{\boldsymbol{\Omega}, \boldsymbol{\Omega}\}$ and we apply the Solution Lemma. We will see below that the generalized skand $\left\{\mathbf{e}_{(0, \omega)},\left\{\mathbf{e}_{(0, \omega)},\{\ldots\}\right\}\right\}$ is not equal to $\mathbf{e}_{(0, \omega)}$.

Analysing AFA we see that a unique solution of a flat equation (13) is actually a proper class of all its solutions in $\mathbf{V}[\mathcal{U}]^{(1)}$. Thus the quotient class $\mathbf{V}[\mathcal{U}]^{(1)}$ recieved by the solution lemma equivalent relation, we obtain a subclass of the hyperset universe. That is why Russell's paradox and other set-theoretical paradoxes (except Cantor's and Hilbert's paradoxes) are possible in the hyperset universe (and only in such systems) of course, on the assumption that $R$ or other considered classes are sets, and, what is especially important, that one has to refer to AFA in implications such as, e.g., $R \notin R \Longrightarrow R \in R$.

\section{8. $\nu$-generalized skands, formal theory $N B G[\mathcal{U}]^{(\nu)}$ and its model} in the universe of elements $\mathbf{U}[\mathcal{U}]^{(\nu)}$, and the universe $\mathbf{U}[\mathcal{U}]^{(\Omega)}$

We have already extended the formal set theory $N B G[\mathcal{U}]^{-}$by a new axiom SEA\&RSS which is less restrictive than FA and have obtained the formal theory $N B G[\mathcal{U}]^{(1)}$ as well as have enriched $\mathbf{V}[\mathcal{U}]=\mathbf{V}[\mathcal{U}]^{(0)}$ by new objects and have extended it to the class $\mathbf{V}[\mathcal{U}]^{(1)}$ of sets $X$ which can be either well-founded or non-well-founded the latter are skands of length $l \geq \omega$ considered as sets of $\mathbf{V}[\mathcal{U}]$. Moreover, we have shown that the class $\mathbf{U}[\mathcal{U}]^{(0)}=\mathbf{V}[\mathcal{U}]^{(0)} \cup \mathcal{U}$ is a model of $N B G[\mathcal{U}]^{(1)}$ and an inner model of $N B G[\mathcal{U}]^{-}$. We can successively continue this process of construction

for each ordinal number $\nu \in \mathbf{O n}$, defining formal theories $N B G[\mathcal{U}]^{\left(\nu^{\prime}\right)}$, $1<\nu^{\prime} \leq \nu$, enriching $\mathbf{V}[\mathcal{U}]^{(0)}$ by new $\nu^{\prime}$-generalized skands, $1<\nu^{\prime} \leq \nu$. Moreover, there are sequential embeddings:

$$
\mathbf{V}[\mathcal{U}]^{(0)} \subset \mathbf{V}[\mathcal{U}]^{(1)} \subset \mathbf{V}[\mathcal{U}]^{(2)} \subset \ldots \subset \mathbf{V}[\mathcal{U}]^{(\nu)} \subset \mathbf{V}[\mathcal{U}]^{-},
$$


where all embeddings are proper.

This can be done by the following transfinite induction.

Suppose that we have already constructed $N B G[\mathcal{U}]^{\left(\nu^{\prime}\right)}$ for each $1 \leq$ $\nu^{\prime}<\nu$, and classes $\mathbf{V}[\mathcal{U}]^{\left(\nu^{\prime}\right)}$ of sets in the coresponding theories, enriching them by $\nu^{\prime \prime}$-generelized skands as sets of $\mathbf{V}[\mathcal{U}]^{-}, 1<\nu^{\prime \prime} \leq \nu^{\prime}$, and hence the universe of elements $\mathbf{U}[\mathcal{U}]^{\left(\nu^{\prime}\right)}=\mathbf{V}[\mathcal{U}]^{\left(\nu^{\prime}\right)} \cup \mathcal{U}$ of this theory $N B G[\mathcal{U}]^{\left(\nu^{\prime}\right)}$. Now we construct a formal set theory $N B G[\mathcal{U}]^{(\nu)}$ and the class $\mathbf{V}[\mathcal{U}]^{(\nu)}$ of all sets in $N B G[\mathcal{U}]^{(\nu)}$ and hence the universe of elements $\mathbf{U}[\mathcal{U}]^{(\nu)}=\mathbf{V}[\mathcal{U}]^{(\nu)} \cup \mathcal{U}$ of this theory $N B G[\mathcal{U}]^{(\nu)}$, and the following sequential embeddings are proper:

$$
\mathbf{V}[\mathcal{U}]=\mathbf{V}[\mathcal{U}]^{(0)} \subset \mathbf{V}[\mathcal{U}]^{(1)} \subset \mathbf{V}[\mathcal{U}]^{(2)} \subset \ldots \subset \mathbf{V}[\mathcal{U}]^{\left(\nu^{\prime}\right)} \subset \mathbf{V}[\mathcal{U}]^{-},
$$

$0 \leq \nu^{\prime}<\nu$.

Definition 5. Let $f_{\left(\alpha_{0}, \alpha\right)}:\left(\alpha_{0}, \alpha\right) \rightarrow \bigcup_{0 \leq \nu^{\prime}<\nu} \mathbf{V}[\mathcal{U}]^{\left(\nu^{\prime}\right)}$ be a map. By a $\nu$-generalized skand $X_{\left(\alpha_{0}, \alpha\right)}^{(\nu)}$ we understand a usual skand as a system of embedded curly braces $\left\{\alpha_{0}\left\{\alpha_{0}+1 \cdots\left\{\alpha^{\prime} \cdots \alpha^{\prime}\right\} \cdots \alpha_{0}+1\right\} \alpha_{0}\right\}$, indexed by ordinals $\alpha^{\prime} \in\left(\alpha_{0}, \alpha\right)$, whose pairs of braces $\{\{$ and \{\} are not filled or filled by previously defined sets of $\bigcup_{0 \leq \nu^{\prime}<\nu} \mathbf{V}[\mathcal{U}]^{\left(\nu^{\prime}\right)}$, i.e., whose components $X_{\alpha^{\prime}}^{(\nu)}$ coincide with the images $f_{\left(\alpha_{0}, \alpha\right)}\left(\alpha^{\prime}\right)$, respectively, $\alpha_{0} \leq \alpha^{\prime}<\alpha$.

Definition 6. Two $\nu$-generalized skands $X_{\left(\alpha_{0}, \alpha\right)}^{(\nu)}$ and $Y_{\left(\beta_{0}, \beta\right)}^{(\nu)}$ are equal if the segments $\left(\alpha_{0}, \alpha\right)$ and $\left(\beta_{0}, \beta\right)$ are isomorphic as well-ordered sets and the corresponding components $X_{\alpha^{\prime}}^{(\nu)}=Y_{\beta^{\prime}}^{(\nu)}$, where this unique isomorphism is given by $\varphi:\left(\alpha_{0}, \alpha\right) \rightarrow\left(\beta_{0}, \beta\right)$ and $\beta^{\prime}=\varphi\left(\alpha^{\prime}\right), \alpha_{0} \leq \alpha^{\prime}<\alpha, \beta_{0} \leq \beta^{\prime}<\beta$, are equal as sets of $\bigcup_{0 \leq \nu^{\prime}<\nu} \mathbf{V}[\mathcal{U}]^{\left(\nu^{\prime}\right)}$.

\section{$\nu$-Generalized Skand Existence Axiom and Recognition $\nu$-Gene- ralized Skand as a Set.}

For any map $f_{\left(\alpha_{0}, \alpha\right)}:\left(\alpha_{0}, \alpha\right) \rightarrow \bigcup_{0 \leq \nu^{\prime}<\nu} \mathbf{V}[\mathcal{U}]^{\left(\nu^{\prime}\right)}, \alpha-\alpha_{0} \geq \omega$, there exists a unique skand $X_{\left(\alpha_{0}, \alpha\right)}^{(\nu)}$ such that for each $\alpha^{\prime} \in\left(\alpha_{0}, \alpha\right)$ one has $X_{\alpha^{\prime}}^{(\nu)}=$ $f_{\left(\alpha_{0}, \alpha\right)}\left(\alpha^{\prime}\right)$. This $\nu$-generalized skand $X_{\left(\alpha_{0}, \alpha\right)}^{(\nu)}$ is considered as a set $X \in$ $\mathbf{V}[\mathcal{U}]^{-}$whose elements are elements of the set $f_{\left(\alpha_{0}, \alpha\right)}\left(\alpha_{0}\right)$ and one more element $X_{\left(\alpha_{0}+1, \alpha\right)}^{(\nu)}$ which is a restriction of $X_{\left(\alpha_{0}, \alpha\right)}^{(\nu)}$ on $\left(\alpha_{0}+1, \alpha\right)$, i.e.,

$$
X=\left\{x_{0}^{\alpha_{0}}, x_{1}^{\alpha_{0}}, \ldots, x_{\lambda}^{\alpha_{0}}, \ldots, X_{\left(\alpha_{0}+1, \alpha\right)}^{(\nu)}\right\},
$$


where $\left\{x_{0}^{\alpha_{0}}, x_{1}^{\alpha_{0}}, \ldots, x_{\lambda}^{\alpha_{0}}, \ldots\right\}$ is a set whose elements are in the class $\bigcup_{0 \leq \nu^{\prime}<\nu} \mathbf{V}[\mathcal{U}]^{\left(\nu^{\prime}\right)}$. The only element of the set $X$ is the skand $X_{\left(\alpha_{0}+1, \alpha\right)}^{(\nu)}$ considered as a set is not an element of $\bigcup_{0 \leq \nu^{\prime}<\nu} \mathbf{V}[\mathcal{U}]^{\left(\nu^{\prime}\right)}$ but an element of $\mathbf{V}[\mathcal{U}]^{-}$.

Notice that $\nu$-genelized skands $X_{\left(\alpha_{0}, \alpha\right)}^{(\nu)}$ of a length $l=\left(\alpha-\alpha_{0}\right)<\omega$ are elements of the class $\bigcup_{0 \leq \nu^{\prime}<\nu} \mathbf{V}[\mathcal{U}]^{\left(\nu^{\prime}\right)}$ and are out of our interest.

As above we will note this very set by the same symbol $X_{\left(\alpha_{0}, \alpha\right)}^{(\nu)}$ but we shall differ $X_{\left(\alpha_{0}, \alpha\right)}^{(\nu)}$ as a $\nu$-generalized skand in Definition 5 and $X_{\left(\alpha_{0}, \alpha\right)}^{(\nu)}$ as a set (32).

As in the case of the first step of induction we want to extend $N B G^{-}$ and $N B G[\mathcal{U}]^{-}$to $N B G^{(\nu)}$ and $N B G[\mathcal{U}]^{(\nu)}$, respectively, by less restrictive axioms than FA, which will be based nevertheless on $N B G$.

As above we suppose that $\mathcal{U}^{(0)}$ is a set (which can be empty) or class, then we will define a theory $N B G[\mathcal{U}]^{(\nu)}$ which is an extension of theories $N B G[\mathcal{U}]^{-}$and $N B G^{-}$; the latter in the case $\mathcal{U}=\emptyset$. For this purpose we add to $N B G^{(0)}$ a wider class of individuals than $\mathcal{U}^{(0)}$ by adding to it a new class of individuals $\mathcal{U}^{(\nu)}$. Elements $u_{\lambda}^{(\nu)} \in \mathcal{U}^{(\nu)}, \lambda \in \mathbf{O n}$, are arbitrary skands $X_{\left(\alpha_{0}, \alpha\right)}^{(\nu)}$ of length $l=\left(\alpha-\alpha_{0}\right) \geq \omega$ taken with the forgetful operator $E$ which "forgets" the inner structure $(\in$ relations $)$ of $X_{\left(\alpha_{0}, \alpha\right)}^{(\nu)}$. More precisely, $u_{\lambda}^{(1)}=E X_{\left(\alpha_{0}, \alpha\right)}^{(\nu)}$ and no element or object is a member of $E X_{\left(\alpha_{0}, \alpha\right)}$. Moreover, if $X_{\left(\alpha_{0}, \alpha\right)}^{(\nu)}=X_{\left(\beta_{0}, \beta\right)}^{(\nu)}$, then $E X_{\left(\alpha_{0}, \alpha\right)}^{(\nu)}=E X_{\left(\beta_{0}, \beta\right)}^{(\nu)}$ and they define the only individual $u_{\lambda}^{(\nu)} \in \mathcal{U}^{(\nu)}$. As above we need the inverse operator, i.e., "remember operator" $E^{-1}$, i.e., for any individual $u_{\lambda}^{(\nu)}=E X_{\left(\alpha_{0}, \alpha\right)}^{(\nu)}, \lambda \in \mathbf{O n}, E^{-1} u_{\lambda}^{(\nu)}=X_{\left(\alpha_{0}, \alpha\right)}^{(\nu)}$.

Since in $N B G[\mathcal{U}]^{(\nu)}$ we postulate existence of new objects and sets we have to revise the previous Extensionality Axiom (sortly EA) of $N B G[\mathcal{U}]^{(0)}$.

\section{$\nu$-Strong Extensionality Axiom.}

Two sets (resp., classes) $X^{(\nu)}$ and $Y^{(\nu)}$ whose elements are well-founded sets, individuals and $\nu$-generalized skands as non-well-founded sets are equal if for each element $x \in X^{(\nu)}$ there is an element $y \in Y^{(\nu)}$ such that $x=y$ and for each element $y \in Y^{(\nu)}$ there is an element $x \in X^{(\nu)}$ such that $y=x$, where "=" means the following: 
1) the equality of individuals, when $x, y \in \mathcal{U}^{(0)}$, i.e., $(x=y) \Longleftrightarrow$ $(\forall z)(x \in z \Longleftrightarrow y \in z)$;

2) the equality of well-founded sets, when $x, y \in \mathbf{V}[\mathcal{U}]^{(0)}$, i.e., by $\mathbf{E A}$ of $N B G[\mathcal{U}]$

3) the equality of $\nu$-generalized skands, when $x, y \in E^{-1} \mathcal{U}^{(\nu)}$, i.e., by Definition 6;

4) the (iterative) equality of sets in $\mathbf{V}[\mathcal{U}]^{(\nu)}$, i.e., by using 1), 2), 3) in $\nu$-SEA for a complex sets $x, y \in \mathbf{V}[\mathcal{U}]^{(\nu)}$.

The latter class $\mathbf{V}[\mathcal{U}]^{(\nu)}$ is defined by transfinite recursion. Denote by $\mathcal{U}^{\prime}$ the discrete union $\mathcal{U}^{(0)} \sqcup \mathcal{U}^{(\nu)}$. By formulas (2) and (3), we define a class $\mathbf{H}\left[\mathcal{U}^{\prime}\right]$ which is a class of well-founded sets and determines a model of $N B G\left[\mathcal{U}^{\prime}\right], \mathbf{H}\left[\mathcal{U}^{\prime}\right]=\mathbf{U}\left[\mathcal{U}^{\prime}\right]$ and is an inner model of $N B G\left[\mathcal{U}^{\prime}\right]^{-}$. Now we apply the remember operator $E^{-1}$ to all individuals of $\mathcal{U}^{(\nu)}$ which are in any constituents of sets in $\mathbf{V}\left[\mathcal{U}^{\prime}\right]=\mathbf{H}\left[\mathcal{U}^{\prime}\right] \backslash \mathcal{U}^{(0)}$ or individuals themselves in $\mathbf{H}\left[\mathcal{U}^{\prime}\right] \backslash \mathcal{U}^{(0)}$, in short words, in all sets $X \in \mathbf{H}\left[\mathcal{U}^{\prime}\right] \backslash \mathcal{U}^{(0)}$ as well as individuals in $\mathcal{U}_{(\nu)} \subset X \in \mathbf{H}\left[\mathcal{U}^{\prime}\right] \backslash \mathcal{U}^{(0)}$ we change all these individuals on the corresponding $\nu$-generalized skands and consider them as sets like in (??).

One can see that after such changing, $\mathbf{V}\left[\mathcal{U}^{\prime}\right]$ turns into the class $\mathbf{V}[\mathcal{U}]^{(\nu)}$ of all sets in this theory $N B G[\mathcal{U}]^{(\nu)}$ which can be well-founded and nonwell-founded. Moreover, by Propositions 1 and $2, \mathbf{V}[\mathcal{U}]^{(\nu)}$ is a model of $N B G[\mathcal{U}]^{(\nu)}$ and an inner model of $N B G[\mathcal{U}]^{-}$.

This construction gives the following theorem.

Theorem 2. The set theory $N B G[\mathcal{U}]^{(\nu)}$ is consistent on the assumption that $N B G^{-}$is consistent.

Proof is the same as in Theorem 1 .

In our case we have an additional class of individuals $\mathcal{U}^{(\nu)}$ as "pseudoindividuals" in the above sense, i.e., each $\in$-descending chain is finite or terminates, i.e., reaches its bottom or "foundation" which is an empty set, or an individual, or a skand, or $\nu^{\prime}$-generalized skand, $1<\nu^{\prime} \leq \nu$.

Since by definitions and construction of $\mathcal{U}^{\prime}$ we obtain the following embeddings:

$$
\mathcal{U}=\mathcal{U}^{(0)} \subset \mathcal{U}^{(1)} \subset \mathcal{U}^{(2)} \subset \ldots \subset \mathcal{U}^{(\nu)}=\mathcal{U}^{\prime} .
$$

It is clear that by (33) and the second part of $\nu$-SEA\&RSS, i.e., $\nu \mathbf{R S S}$, we obtain the following embeddings:

$$
\mathbf{V}[\mathcal{U}]=\mathbf{V}[\mathcal{U}]^{(0)} \subset \mathbf{V}[\mathcal{U}]^{(1)} \subset \mathbf{V}[\mathcal{U}]^{(2)} \subset \ldots \subset \mathbf{V}[\mathcal{U}]^{(\nu)} \subset \mathbf{V}[\mathcal{U}]^{-} .
$$

Note that the latter embedding is proper because of the possibility of the next step $\nu+1$ of trasfinite induction. 
At last, since we have constructed $N B G[\mathcal{U}]^{(\nu)}$ and $\mathbf{V}[\mathcal{U}]^{(\nu)}$ for all $\nu \in \mathbf{O n}$ we obtain the following proper embeddings:

$\mathbf{V}[\mathcal{U}]=\mathbf{V}[\mathcal{U}]^{(0)} \subset \mathbf{V}[\mathcal{U}]^{(1)} \subset \mathbf{V}[\mathcal{U}]^{(2)} \subset \ldots \subset \mathbf{V}[\mathcal{U}]^{(\nu)} \subset \ldots \subset \mathbf{V}[\mathcal{U}]^{(\Omega)} \subset \mathbf{V}[\mathcal{U}]^{-}$

where $\mathbf{V}[\mathcal{U}]^{(\Omega)}=\bigcup_{\nu \in \mathbf{O} \mathbf{n}} \mathbf{V}[\mathcal{U}]^{(\nu)}$ and the formal non-well-founded sets theory $N B G[\mathcal{U}]^{(\Omega)}$ has all axioms of $N B G[\mathcal{U}]^{-}$plus two additional axioms. We shall start with the following definitions

Definition 7. Let $f_{\left(\alpha_{0}, \alpha\right)}:\left(\alpha_{0}, \alpha\right) \rightarrow \mathbf{V}[\mathcal{U}]^{(\Omega)}$ be a map. By a $\Omega$ generalized skand $X_{\left(\alpha_{0}, \alpha\right)}^{(\Omega)}$ we understand a usual skand as a system of embedded curly braces $\left\{\alpha_{0}\left\{\alpha_{0}+1 \cdots\left\{\alpha^{\prime} \cdots \alpha^{\prime}\right\} \cdots \alpha_{0}+1\right\} \alpha_{0}\right\}$, indexed by ordinals $\alpha^{\prime} \in\left(\alpha_{0}, \alpha\right)$, whose pairs of braces $\{\{$ and \{\} are not filled or filled by sets of $\mathbf{V}[\mathcal{U}]^{(\Omega)}$, i.e., whose components $X_{\alpha^{\prime}}^{(\Omega)}$ coincide with the images $f_{\left(\alpha_{0}, \alpha\right)}\left(\alpha^{\prime}\right)$, $\alpha_{0} \leq \alpha^{\prime}<\alpha$.

Definition 8. Two $\Omega$-generalized skands $X_{\left(\alpha_{0}, \alpha\right)}^{(\Omega)}$ and $Y_{\left(\beta_{0}, \beta\right)}^{(\Omega)}$ are equal if the segments $\left(\alpha_{0}, \alpha\right)$ and $\left(\beta_{0}, \beta\right)$ are isomorphic as well-ordered sets and the corresponding components $X_{\alpha^{\prime}}^{(\Omega)}=Y_{\beta^{\prime}}^{(\Omega)}$, where this unique isomorphism is given by $\varphi:\left(\alpha_{0}, \alpha\right) \rightarrow\left(\beta_{0}, \beta\right)$ and $\beta^{\prime}=\varphi\left(\alpha^{\prime}\right), \alpha_{0} \leq \alpha^{\prime}<\alpha, \beta_{0} \leq \beta^{\prime}<\beta$, are equal as sets of $\mathbf{V}[\mathcal{U}]^{(\Omega)}$.

$\Omega$-Generalized Skand Existence Axiom and Recognition $\Omega$-Generalized Skand as a Set.

For any map $f_{\left(\alpha_{0}, \alpha\right)}:\left(\alpha_{0}, \alpha\right) \rightarrow \mathbf{V}[\mathcal{U}]^{(\Omega)}, \alpha-\alpha_{0} \geq \omega$, there exists a unique skand $X_{\left(\alpha_{0}, \alpha\right)}^{(\Omega)}$ such that for each $\alpha^{\prime} \in\left(\alpha_{0}, \alpha\right)$ one has $X_{\alpha^{\prime}}^{(\Omega)}=$ $f_{\left(\alpha_{0}, \alpha\right)}\left(\alpha^{\prime}\right)$. This $\Omega$-generalized skand $X_{\left(\alpha_{0}, \alpha\right)}^{(\Omega)}$ is considered as a set $X \in$ $\mathbf{V}[\mathcal{U}]^{-}$whose elements are elements of the set $f_{\left(\alpha_{0}, \alpha\right)}\left(\alpha_{0}\right)$ and one more element $X_{\left(\alpha_{0}+1, \alpha\right)}^{(\Omega)}$ which is a restriction of $X_{\left(\alpha_{0}, \alpha\right)}^{(\Omega)}$ on $\left(\alpha_{0}+1, \alpha\right)$, i.e.,

$$
X=\left\{x_{0}^{\alpha_{0}}, x_{1}^{\alpha_{0}}, \ldots, x_{\lambda}^{\alpha_{0}}, \ldots, X_{\left(\alpha_{0}+1, \alpha\right)}^{(\Omega)}\right\}
$$

where $\left\{x_{0}^{\alpha_{0}}, x_{1}^{\alpha_{0}}, \ldots, x_{\lambda}^{\alpha_{0}}, \ldots, X_{\left(\alpha_{0}+1, \alpha\right)}^{(\Omega)}\right\}$ is a set whose elements are mebmers of the class $\mathbf{V}[\mathcal{U}]^{(\Omega)}$.

Notice that in this limit case each $\Omega$-generalized skand $X_{\left(\alpha_{0}, \alpha\right)}^{(\Omega)}, \alpha-\alpha_{0} \geq$ $\omega$, coincides with $\nu$-generalized skand $X_{\left(\alpha_{0}, \alpha\right)}^{(\nu)}$ for some ordinal $\nu \in$ On and thus $X_{\left(\alpha_{0}+1, \alpha\right)}^{(\Omega)}$ is not a new element which should be enreach $\mathbf{V}[\mathcal{U}]^{(\Omega)}$. Indeed, it is a consequence of the following proposition. 
Proposition 16. For any $\Omega$-generalized skand $X_{\left(\alpha_{0}, \alpha\right)}^{(\Omega)}$ there exists a minimal ordinal $\nu$ and a unique $\nu$-generalized skand $X_{\left(\alpha_{0}, \alpha\right)}^{(\nu)}$ such that for each $\alpha^{\prime} \in\left(\alpha_{0}, \alpha\right)$ one has $X_{\alpha^{\prime}}^{(\Omega)}=X_{\alpha^{\prime}}^{(\nu)}$.

Proof. Since, by Definition 7, each $X_{\alpha^{\prime}}^{(\Omega)}$ is a set and $X_{\alpha^{\prime}}^{(\Omega)}=f_{\left(\alpha_{0}, \alpha\right)}\left(\alpha^{\prime}\right)$, $\alpha_{0} \leq \alpha^{\prime}<\alpha$, and $X_{\alpha^{\prime}}^{(\Omega)} \in \mathbf{V}[\mathbf{U}]^{(\Omega)}=\bigcup_{\nu^{\prime} \in \mathbf{O} \mathbf{n}} X^{\left(\nu^{\prime}\right)}$ we can find a minimal ordinal $\nu_{\alpha^{\prime}}^{\prime}$ such that $f_{\left(\alpha_{0}, \alpha\right)}\left(\alpha^{\prime}\right) \in \mathbf{V}[\mathcal{U}]^{\left(\ni_{\alpha^{\prime}}^{\prime}\right)}$. Indeed, $\mathbf{V}[\mathcal{U}]^{\left(\ni_{\alpha^{\prime}}^{\prime}\right)}$ is the intersection of all $\mathbf{V}[\mathcal{U}]^{\left(\ni^{\prime \prime}\right)}$ such that $f_{\left(\alpha_{0}, \alpha\right)}\left(\alpha^{\prime}\right) \in \mathbf{V}[\mathcal{U}]^{\left(\ni^{\prime \prime}\right)}$. Since $\left\{\nu_{\alpha_{0}}, \nu_{\alpha_{0}+1}, \ldots, \nu_{\alpha^{\prime}}, \ldots\right\}$ is a set which contains $\alpha$ ordinals we can take $\nu^{\prime \prime}=$ $\sup \nu_{\alpha^{\prime}}^{\prime}$ which always exists. Then putting $\nu=\nu^{\prime \prime}+1$, we obtain the $\alpha^{\prime} \in\left(\alpha_{0}, \alpha\right)$

conclusion of Proposition 16.

Moreover, $\Omega$-GSEA\&R $\Omega$-GSS is the weakest restriction axiom among all previous axioms FA, SEA $\&$ RSS, 1-SEA $\&$ RSS, ..., -SEA $\&$ RSS,,. $1<\nu \in \mathbf{O n}$. Hence $\mathbf{V}[\mathcal{U}]^{(\Omega)}$ is the largest class of wel-founded and nonwell-founded sets in the theory $N B G[\mathbf{U}]^{(\Omega)}$. We also denote by $\mathbf{U}[\mathcal{U}]^{(\Omega)}=$ $\mathbf{V}[\mathcal{U}]^{(\Omega)} \cup \mathcal{U}$ the universe of elements or as usually say "the universe of arguments".

$\Omega$-Strong Extensionality Axiom. Two sets (resp., classes) $X^{(\Omega)}$ and $Y^{(\Omega)}$ whose elements are well-founded sets, individuals and $\Omega$-generalized skands as non-well-founded sets are equal if for each element $x \in X^{(\Omega)}$ there is an element $y \in Y^{(\Omega)}$ such that $x=y$ and for each element $y \in Y^{(\Omega)}$ there is an element $x \in X^{(\Omega)}$ such that $y=x$, where "=" means the following:

1) the equality of individuals, when $x, y \in \mathcal{U}^{(0)}$, i.e., $(x=y) \Longleftrightarrow$ $(\forall z)(x \in z \Longleftrightarrow y \in z)$;

2) the equality of well-founded sets, when $x, y \in \mathbf{V}[\mathcal{U}]^{(0)}$, i.e., by $\mathbf{E A}$ of $N B G[\mathcal{U}]$;

3) the equality of $\Omega$-generalized skands, when $x, y \in E^{-1} \mathcal{U}^{(\Omega)}$, i.e., by Definition 8;

4) the (iterative) equality of sets in $\mathbf{V}[\mathcal{U}]^{(\Omega)}$, i.e., by using 1$\left.\left.), 2\right), 3\right)$ in $\Omega$-SEA for a complex sets $x, y \in \mathbf{V}[\mathcal{U}]^{(\Omega)}$.

Theorem 3. The set theory $N B G[\mathcal{U}]^{(\Omega)}$ is consistent on the assumption that $N B G^{-}$is consistent.

Proof is the same as in Theorem 1.

In our case we have an additional class of individuals $\mathcal{U}^{(\nu)}$ as "pseudoindividuals" in the above sense, i.e., each $\in$-descending chain is finite or terminates, i.e., reaches its bottom or "foundation" which is an empty set, or an individual, or a skand, or $\nu$-generalized skand, for some $1<\nu \in \mathbf{O n}$. 


\section{Skand operations and categories of generalized skands}

Let $\alpha \geq \omega$ be an arbitrary fixed ordinal number. We consider now all $\Omega$-generalized skands $X_{(0, \alpha)}^{(\Omega)}$ whose components $X_{\alpha^{\prime}}^{(\Omega)}, 0 \leq \alpha^{\prime}<\alpha$, are elements of $\mathbf{U}^{(\Omega)}=\mathbf{V}^{(\Omega)}$, i.e., we consider the formal non-well-founded set theory $N B G^{(\Omega)}$ without individuals, i.e., $\mathcal{U}=\emptyset$ or, in other words, the theory of "pures" sets. (For simplicity, we omit the index $(\Omega)$ and write $X_{(0, \alpha)}$ instead of $X_{(0, \alpha)}^{(\Omega)}, X_{\alpha^{\prime}}$ instead of $X_{\alpha^{\prime}}^{(\Omega)}$, and generalized skand instead of $\Omega$-generalized skand.)

It is clear, that all such skands form a class (proper class), not a set. We denote it here by $\mathbf{S}^{(\alpha)}$. Inside $\mathbf{S}^{(\alpha)}$ we define the following so called skand-operations.

Definition 9. By the union $X_{(0, \alpha)} \cup Y_{(0, \alpha)}$, intersection $X_{(0, \alpha)} \cap Y_{(0, \alpha)}$, difference $X_{(0, \alpha)} \backslash Y_{(0, \alpha)}$ of two elements $X_{(0, \alpha)}$ and $Y_{(0, \alpha)}$ of $\mathbf{S}^{(\alpha)}$ we understand a generalized skand $Z_{(0, \alpha)}$ whose components $Z_{\alpha^{\prime}}$ are $X_{\alpha^{\prime}} \cup Y_{\alpha^{\prime}}$, $X_{\alpha^{\prime}} \cap Y_{\alpha^{\prime}}$ and $X_{\alpha^{\prime}} \backslash Y_{\alpha^{\prime}}$, respectively, $0 \leq \alpha^{\prime}<\alpha$. By the power of a generalized skand $X_{(0, \alpha)}$ we understand a generalized skand $\mathcal{P} X_{(0, \alpha)}$ whose components $(\mathcal{P} X)_{\alpha^{\prime}}$ are $\mathcal{P} X_{\alpha^{\prime}}$.

Definition 10. By the mapping $f_{(0, \alpha)}: X_{(0, \alpha)} \rightarrow Y_{(0, \alpha)}$ of two generalized skands $X_{(0, \alpha)}$ and $Y_{(0, \alpha)}$ we understand a generalized skand $f_{(0, \alpha)}$ whose components $f_{\alpha^{\prime}}$ are the mappings $f_{\alpha^{\prime}}: X_{\alpha^{\prime}} \rightarrow Y_{\alpha^{\prime}}$, where $X_{\alpha^{\prime}}$ and $Y_{\alpha^{\prime}}$ are components of $X_{(0, \alpha)}$ and $Y_{(0, \alpha)}$, respectively, $0 \leq \alpha^{\prime}<\alpha$. Moreover, $f_{(0, \alpha)}$ is an injection, surjection and bijection, respectively, if the same are all mappings $f_{\alpha^{\prime}}, 0 \leq \alpha^{\prime}<\alpha$. In particular, a generalized skand $X_{(0, \alpha)}$ is a subskand of $Y_{(0, \alpha)}$, if there exists an identical injection $1_{(0, \alpha)}: X_{(0, \alpha)} \rightarrow Y_{(0, \alpha)}$.

It is clear that one can define other skand-theoretical operations such as products and coproducts, inverse and direct limits, pull-backs and pushouts, the equivalence relation and quotients, etc., by the set-operations on the corresponding components of skands, respectively. We shall not use these constructions here, and thus omit details.

One can easily verify that the elements of $\mathbf{S}^{(\alpha)}$ as objects and mappings between such objects as morphisms form a category which we denote by $\mathbf{S k}^{(\alpha)}$.

Of special interest for us here are categories $\mathbf{S k}^{\left(\omega^{\kappa}\right)}(P), \kappa \geq 0$, whose objects are self-similar generalized skands $X_{(0, \alpha)}(X)$, i.e., when $\alpha=\omega^{\kappa}$, $\kappa \geq 0$ is fixed, and whose components are permanent, i.e., $X_{\alpha^{\prime}}=X$, for each $0 \leq \alpha^{\prime}<\alpha, X \in \mathbf{V}[\mathcal{U}]^{(\Omega)}$ and whose morphisms are self-similar generalized skand-mappings $f_{(0, \alpha)}(f)$ of such generalized skands whose components are permanent, i.e., mappings $f_{\alpha^{\prime}}=f: X \rightarrow Y$, for each $0 \leq \alpha^{\prime}<\alpha$.

For $\kappa=0$, we consider the usual category of sets whose objects are elements of $\mathbf{V}[\mathcal{U}]^{(\Omega)}$; i.e., sets and morphisms are mappings of sets. 
Proposition 17. For each $X_{\left(0, \omega^{\kappa}\right)}(X) \in \mathbf{S k}^{\left(\omega^{\kappa}\right)}(P), \kappa \geq 1$, there is an index $\lambda, 1 \leq \lambda$, such that $X_{\left(0, \omega^{\kappa}\right)}(X) \in \mathcal{U}^{(\lambda)}$.

Proof. Since $X \in \mathbf{V}[\mathcal{U}]^{(\Omega)}$ there exists an ordinal number $\nu \geq 0$ such that $X \in \mathbf{V}[\mathcal{U}]^{(\nu)}$. Among all such $\nu$ there is a minimal number and we denote it also by $\nu$. Then $X_{\left(0, \omega^{\kappa}\right)}(X) \in \mathbf{V}[\mathcal{U}]^{(\nu+1)}$ and we put $\lambda=\nu+1$.

It is clear that all skand-operations on self-similar generalized skands give us a self-similar generalized skand as a result. We shall often use the following skand-operation:

Definition 9. Let $X_{\left(0, \omega^{\kappa}\right)}(X)\left(X_{\left(0, \omega^{\kappa}\right)}\right.$ for short) be a self-similar generalized skand, where $\kappa \geq 1$ is fixed. By a singleton-skand we understand the following self-similar generalized skand $S_{\left(0, \omega^{\kappa}\right)}\left(X_{\left(0, \omega^{\kappa}\right)}\right)=\left\{X_{\left(0, \omega^{\kappa}\right)},\left\{X_{\left(0, \omega^{\kappa}\right)},\{\ldots\}\right\}\right\}$.

It is clear that, by Definition 6 , the empty skand $\mathbf{e}_{\left(0, \omega^{\kappa}\right)}$ is not equal to the singleton-skand $S_{\left(0, \omega^{\kappa}\right)}\left(\mathbf{e}_{\left(0, \omega^{\kappa}\right)}\right)$ in contrast to their equality in $\mathbf{Z F A}^{-}+\mathbf{A F A}$ (see [1], p. 8) as we promised to show in paragraph 7 .

There is an evident functor $F_{\kappa^{\prime} \kappa^{\prime \prime}}: \mathbf{S k}^{\left(\omega^{\kappa^{\prime}}\right)}(P) \rightarrow \mathbf{S k}^{\left(\omega^{\kappa^{\prime \prime}}\right)}(P), 1 \leq \kappa^{\prime} \leq$ $\kappa^{\prime \prime}$, which associates with every object $X_{\left(0, \omega^{\kappa^{\prime}}\right)}(X)$ the object $X_{\left(0, \omega^{\kappa^{\prime \prime}}\right)}(X)$ and with every self-similar morphism $f_{\left(0, \omega^{\kappa^{\prime}}\right)}(f): X_{\left(0, \omega^{\kappa^{\prime}}\right)}(X) \rightarrow Y_{\left(0, \omega^{\kappa^{\prime}}\right)}(Y)$ the self-similar morphism $f_{\left(0, \omega^{\kappa^{\prime \prime}}\right)}(f): X_{\left(0, \omega^{\kappa^{\prime \prime}}\right)}(X) \rightarrow Y_{\left(0, \omega^{\kappa^{\prime \prime}}\right)}(Y)$. A verification of the category's axioms is trivial. Moreover, all categories $\mathbf{S k}^{\left(\omega^{\kappa}\right)}(P)$, $\kappa \geq 1$, are isomorphic to each other and hence to the usual category of sets, which are elements of $\mathbf{U}^{(\Omega)}$ and morphisms are maps of such sets.

Proposition 18. The skand $\mathbf{V}_{\left(0, \omega^{\kappa}\right)}$ which is a skand-union of all selfsimilar generalized skands of length $\omega^{\kappa}$, i.e., $\mathbf{V}_{\left(0, \omega^{\kappa}\right)}=\bigcup_{X \in \mathbf{V}[\mathcal{U}]^{(\Omega)}} X_{\left(0, \omega^{\kappa}\right)}(X)$, where $\kappa \geq 1$ and fixed, is not an element of $\mathbf{V}[\mathcal{U}]^{(\Omega)}$; i.e., it is a proper class, not a set.

Proof. Suppose the contrary, and $\mathbf{V}_{\left(0, \omega^{\kappa}\right)}$ is a set. Consequently, there exists a generalized singleton-skand $\mathbf{S}_{\left(0, \omega^{\kappa}\right)}\left(\mathbf{V}_{\left(0, \omega^{\kappa}\right)}\right)$ of $\mathbf{V}_{\left(0, \omega^{\kappa}\right)}$ which is a set, too. Thus, there exists a singleton skand $\mathbf{S}_{\left(0, \omega^{\kappa}\right)}\left(\mathbf{S}_{\left(0, \omega^{\kappa}\right)}\left(\mathbf{V}_{\left(0, \omega^{\kappa}\right)}\right)\right)$. Since $\mathbf{S}_{\left(0, \omega^{\kappa}\right)}\left(\mathbf{V}_{\left(0, \omega^{\kappa}\right)}\right)$ is not an element of a permanent component of $\mathbf{V}_{\left(0, \omega^{\kappa}\right)}$ (otherwise, we should have a skand of the form

$\mathbf{V}_{\left(0, \omega^{\kappa}\right)}=\left\{a, b, c, \ldots,\left\{\mathbf{V}_{\left(0, \omega^{\kappa}\right)}\right\}, \mathbf{V}_{\left(0, \omega^{\kappa}\right)}\right\}$ which is impossible) we obtain that $\mathbf{V}_{\left(0, \omega^{\kappa}\right)}$ is a proper subskand of $\mathbf{V}_{\left(0, \omega^{\kappa}\right)} \cup \mathbf{S}_{\left(0, \omega^{\kappa}\right)}\left(\mathbf{S}_{\left(0, \omega^{\kappa}\right)}\left(\mathbf{V}_{\left(0, \omega^{\kappa}\right)}\right)\right)$ which is in contradiction with the maximality of $\mathbf{V}_{\left(0, \omega^{\kappa}\right)}$. Consequently, $\mathbf{V}_{\left(0, \omega^{\kappa}\right)}$ is not a set, but a proper class, and there is no a generalized singleton-skand $\mathbf{S}_{\left(0, \omega^{\kappa}\right)}\left(\mathbf{V}_{\left(0, \omega^{\kappa}\right)}\right)$ of it, which is of a great importance for us.

\section{A new representation of ordinal and cardinal numbers}


We recall once more that, due to Cantor, an ordinal number $\alpha$ (shortly an ordinal or an element of On) is "the ordinal type of a well-ordered set" [34], p. 152. Likewise, a cardinal number $\aleph_{\alpha}$ (shortly a cardinal or an element of Card) was defined by Cantor as "the power type of equivalent sets" [34], p. 87. In other words, an ordinal number $\alpha$ is a common symbol for the class of all isomorphic ("similar") well-ordered sets and a cardinal number $\left(n \geq 0\right.$, for natural or finite cardinal numbers and $\aleph_{\nu}, \nu \in \mathbf{O n}$, $\nu \geq 0$, for transfinite or infinite cardinal numbers) is a common symbol for the class of equivalent sets, i.e., which are into a one-to-one correspondence. There is a natural binary relation $<$ between two ordinals (respectively, cardinals) $\alpha$ and $\beta$ iff there exist well-ordered sets (respectively, sets) $A$ and $B$ of the ordinal (respectively, cardinal) types $\alpha$ and $\beta$, respectively, and an initial segment $B^{\prime} \subset B$ such that $A$ and $B^{\prime}$ are similar (respectively, equivalent). (Note that we identify here cardinal numbers with the natural and initial ordinal numbers.)

There are diffferent represenations of ordinals numbers as special wellfounded well-ordered sets, e.g., initial segments $(0, \alpha)$ of On ordered by inclusion (Cantor [15]); the canonical representation of ordinals by pure sets $\emptyset,\{\emptyset\},\{\emptyset,\{\emptyset\}\},\{\emptyset,\{\emptyset\},\{\emptyset,\{\emptyset\}\}\}$, etc. (Mirimanoff $[43]$ ); the represenation of ordinal numbers by well-founded sets which are ordered by $\in$ and transitive (von Neumann [49]). There is a series of similar constructions which represent the class of all ordinal numbers (and hence all cardinal numbers) by non-well-founded sets which are well-ordered by $\in$ and transitive. The state of affairs is that these constructions give one more detail concerning the last ordinal and cardinal number, called here the eschaton.

Definition 10. A reflexive set (in particular, a self-similar skand) $\alpha$, which elements are also reflective sets, is called an ordinal number if it is reflexively well-ordered by the relation $\in$ between its elements, i.e., for $X, Y \in \alpha, X \in Y$ includes the case $X=Y$ since $X \in X$; moreover, it is transitive, i.e., if $X, Y, Z \in \alpha$ and $Z \in Y \in X$, then $Z \in X$; it satisfies the following conditions: 1) for every $X, Y \in \alpha$, if $X \neq Y$, then either $X \in Y$ or $Y \in X$, but not both; 2) every non-empty subset of $\alpha$ has a minimal element. Two ordinal numbers $\alpha$ and $\beta$ are equal if there exists a bijection between them, which is an order-preserving function.

Remark 14. Definition 10 differs from the analogous classical definition of an ordinal number in $N B G$ because in the latter case sets $X, Y, Z$ are well-founded, as opposite to the former; one more remark: if $\alpha \in \beta$, then $\alpha<\beta$ and there is no relation $\alpha \in \alpha$ in the classical case, contrary to Definition 10, where $\alpha \in \beta$ implies in general $\alpha \leq \beta$, since an equality may exist in the case $\beta=\alpha$. One can compare the definition of a reflexive 
well-ordering with a reflexive total ordering $R$ in [41], p. 9 , such that every non-empty subset of the field of $R$ has an $R$-least element.

The classes $\mathbf{O n}_{\omega^{\kappa}}, \kappa \geq 1$, of all ordinals in the sense of Definition 10 can be defined by the following transfinite induction.

Let $\kappa>0$ be a fixed usual ordinal number, i.e., an element of $\mathbf{O n}$. We begin with the empty skand $\mathbf{e}_{\left(0, \omega^{\kappa}\right)}=\{\{\{\ldots\}\}\}$ and call it the first element of $\mathbf{O n}_{\omega^{\kappa}}$, denoting it by $\mathbf{e}^{(1)}$. Using Definition 9, we put successively

$$
\begin{aligned}
& \mathbf{e}^{(1)}=\mathbf{e}_{\left(0, \omega^{\kappa}\right)} \\
& \mathbf{e}^{(2)}=\left\{\mathbf{e}^{(1)},\left\{\mathbf{e}^{(1)},\{\ldots\}\right\}\right\}=\left\{\mathbf{e}^{(1)}, \mathbf{e}^{(2)}\right\}, \\
& \mathbf{e}^{(3)}=\left\{\mathbf{e}^{(1)}, \mathbf{e}^{(2)},\left\{\mathbf{e}^{(1)}, \mathbf{e}^{(2)},\{\ldots\}\right\}\right\}=\left\{\mathbf{e}^{(1)}, \mathbf{e}^{(2)}, \mathbf{e}^{(3)}\right\}, \\
& \ldots \ldots \ldots \ldots \ldots \ldots \ldots \ldots \ldots \ldots \ldots \ldots \ldots \ldots \ldots \\
& \mathbf{e}^{(n)}=\left\{\mathbf{e}^{(1)}, \mathbf{e}^{(2)}, \ldots, \mathbf{e}^{(n-1)},\left\{\mathbf{e}^{(1)}, \mathbf{e}^{(2)}, \ldots, \mathbf{e}^{(n-1)},\{\ldots\}\right\}=\right. \\
& \quad=\left\{\mathbf{e}^{(1)}, \mathbf{e}^{(2)}, \ldots, \mathbf{e}^{(n-1)}, \mathbf{e}^{(n)}\right\}, \\
& \quad \mathbf{e}^{(\omega)}=\left\{\mathbf{e}^{(1)}, \mathbf{e}^{(2)}, \mathbf{e}^{(3)}, \ldots,\left\{\mathbf{e}^{(1)}, \mathbf{e}^{(2)}, \mathbf{e}^{(3)}, \ldots,\{\ldots\}\right\}=\right. \\
& \quad=\left\{\mathbf{e}^{(1)}, \mathbf{e}^{(2)}, \mathbf{e}^{(3)}, \ldots, \mathbf{e}^{(\omega)}\right\}, \\
& \mathbf{e}^{(\omega+1)}=\left\{\mathbf{e}^{(1)}, \mathbf{e}^{(2)}, \mathbf{e}^{(3)}, \ldots, \mathbf{e}^{(\omega)},\left\{\mathbf{e}^{(1)}, \mathbf{e}^{(2)}, \mathbf{e}^{(3)}, \ldots, \mathbf{e}^{(\omega)},\{\ldots\}\right\}\right\}= \\
& \left\{\mathbf{e}^{(1)}, \mathbf{e}^{(2)}, \mathbf{e}^{(3)}, \ldots, \mathbf{e}^{(\omega)}, \mathbf{e}^{(\omega+1)}\right\},
\end{aligned}
$$

It is clear that

$\mathbf{e}^{(1)} \in \mathbf{e}^{(1)}$ and $\mathbf{e}^{(1)} \subseteq \mathbf{e}^{(1)}, \mathbf{e}^{(1)} \in \mathbf{e}^{(2)}$ and $\mathbf{e}^{(1)} \subset \mathbf{e}^{(2)}, \mathbf{e}^{(1)} \in \mathbf{e}^{(3)}$ and $\mathbf{e}^{(1)} \subset \mathbf{e}^{(3)}, \ldots \mathbf{e}^{(1)} \in \mathbf{e}^{(\omega)}$ and $\mathbf{e}^{(1)} \subset \mathbf{e}^{(\omega)}, \ldots ;$

$\mathbf{e}^{(2)} \in \mathbf{e}^{(2)}$ and $\mathbf{e}^{(2)} \subseteq \mathbf{e}^{(2)}, \mathbf{e}^{(2)} \in \mathbf{e}^{(3)}$ and $\mathbf{e}^{(2)} \subset \mathbf{e}^{(3)}, \mathbf{e}^{(2)} \in \mathbf{e}^{(4)}$ and $\mathbf{e}^{(2)} \subset \mathbf{e}^{(4)}, \ldots \mathbf{e}^{(2)} \in \mathbf{e}^{(\omega)}$ and $\mathbf{e}^{(2)} \subset \mathbf{e}^{(\omega)}, \ldots ;$

and so forth.

One can see that each ordinal $\mathbf{e}^{(\alpha)}$ is the ordinal type of the 0 -component $\mathbf{e}_{0}^{(\alpha)}$ of the skand $\mathbf{e}^{(\alpha)}$. Moreover, although there is no the ordinal number 0 among self-similar skands, there is a one-to-one correspondence between the class of all ordinals $\mathbf{O n}_{\omega^{\kappa}}=\left\{\mathbf{e}^{(1)}, \mathbf{e}^{(2)}, \ldots, \mathbf{e}^{(\alpha)}, \ldots\right\}, \alpha \geq 1$, and the class of all $\alpha \in \mathbf{O n}$, because the ordinal type of 0 -component $\mathbf{e}^{(\alpha)}$, which is not a self-similar skand, is the same as the initial segment $(0, \alpha)$ of On.

Consider now a more interesting description of the class of all ordinals in the sense of Definition 10. It is the skand-union of all ordinal numbers, i.e., $\boldsymbol{\Omega}_{\left(0, \omega^{\kappa}\right)}=\left\{\bigcup_{\alpha \geq 1} \mathbf{e}^{(\alpha)},\left\{\bigcup_{\alpha \geq 1} \mathbf{e}^{(\alpha)},\{\ldots\}\right\}\right\}$.

Proposition 19. The self-similar skand $\boldsymbol{\Omega}_{\left(0, \omega^{\kappa}\right)}$, where $\kappa \geq 1$ and is fixed, is not an element of $\mathbf{V}[\mathcal{U}]^{(\Omega)}$; i.e., it is a proper class, not a set.

Proof. Suppose the contrary, and $\boldsymbol{\Omega}_{\left(0, \omega^{\kappa}\right)}$ is a set. Consequently, there exists a generalized singleton-skand $S_{\left(0, \omega^{\kappa}\right)}\left(\boldsymbol{\Omega}_{\left(0, \omega^{\kappa}\right)}\right)=\left\{\boldsymbol{\Omega}_{\left(0, \omega^{\kappa}\right)},\left\{\boldsymbol{\Omega}_{\left(0, \omega^{\kappa}\right)},\{\ldots\}\right\}\right\}$ of $\boldsymbol{\Omega}_{\left(0, \omega^{\kappa}\right)}$ which is a set, too. 
Thus, there exists a singleton skand

$S_{\left(0, \omega^{\kappa}\right)}\left(S_{\left(0, \omega^{\kappa}\right)}\left(\boldsymbol{\Omega}_{\left(0, \omega^{\kappa}\right)}\right)\right)$. Since $S_{\left(0, \omega^{\kappa}\right)}\left(\boldsymbol{\Omega}_{\left(0, \omega^{\kappa}\right)}\right)$ is not an element of a permanent component of $\boldsymbol{\Omega}_{\left(0, \omega^{\kappa}\right)}$ (otherwise, we should have a skand of the form $\omega_{\left(0, \omega^{\kappa}\right)}=\left\{a, b, c, \ldots,\left\{\boldsymbol{\Omega}_{\left(0, \omega^{\kappa}\right)}\right\}, \boldsymbol{\Omega}_{\left(0, \omega^{\kappa}\right)}\right\}$ which is impossible) we obtain that $\boldsymbol{\Omega}_{\left(0, \omega^{\kappa}\right)}$ is a proper subskand of $\boldsymbol{\Omega}_{\left(0, \omega^{\kappa}\right)} \cup S_{\left(0, \omega^{\kappa}\right)}\left(S_{\left(0, \omega^{\kappa}\right)}\left(\boldsymbol{\Omega}_{\left(0, \omega^{\kappa}\right)}\right)\right)$ which is in contradiction with the maximality of $\boldsymbol{\Omega}_{\left(0, \omega^{\kappa}\right)}$. Consequently, $\boldsymbol{\Omega}_{\left(0, \omega^{\kappa}\right)}$ is not a set, but a proper class, and there is not a generalized singleton-skand $S_{\left(0, \omega^{\kappa}\right)}\left(\boldsymbol{\Omega}_{\left(0, \omega^{\kappa}\right)}\right)$ of it, which is of great importance for us.

Thus $\boldsymbol{\Omega}_{\left(0, \omega^{\kappa}\right)}$ with a fixed $\kappa \geq 1$ is a generalized skand-class whose permanent component is a well-ordered class $\mathbf{O n}_{\omega^{\kappa}}$.

Moreover, formally $\boldsymbol{\Omega}_{\left(0, \omega^{\kappa}\right)} \in \boldsymbol{\Omega}_{\left(0, \omega^{\kappa}\right)}$, which is not in contradiction with an agreement that classes are not elements of classes. In our case, with a specific definition of skand-operations, $\boldsymbol{\Omega}_{\left(0, \omega^{\kappa}\right)}$ cannot be an element of any class or set, e.g., the singleton $S_{\left(0, \omega^{\kappa}\right)}\left(\boldsymbol{\Omega}_{\left(0, \omega^{\kappa}\right)}\right)$ which does not exist at all as an element of $\mathbf{V}[\mathcal{U}]^{(\Omega)}$, but, by our natural construction, it is an element of itself.

We see also that $\boldsymbol{\Omega}_{\left(0, \omega^{\kappa^{\prime}}\right)}$ and $\boldsymbol{\Omega}_{\left(0, \omega^{\kappa^{\prime \prime}}\right)}$ are isomorphic for every $1 \leq \kappa^{\prime}<$ $\kappa^{\prime \prime}$ and we can omit indexes; i.e., we write $\boldsymbol{\Omega}$ and call this generalized skandclass the last ordinal number or the eschaton in the sense of Definition 10, because it is well-ordered by $\in$, and is class-transitive. In other words, $\boldsymbol{\Omega}$ is a common symbol for the ordinal type of well-ordered proper classes whose all initial segments are sets. It is the last, indeed, because there are no more units, i.e., generalized singleton-skands one could add to $\boldsymbol{\Omega}$.

It is clear that $\boldsymbol{\Omega}$ is the initial class-ordinal number because it is not equinumerous to any smaller ordinal number. Indeed, any $\alpha<\boldsymbol{\Omega}$ is a set and hence is not equivalent to $\Omega$. By definition, the cardinality $|A|$ of any proper class $A$ is defined as the unique class-cardinal $\boldsymbol{\Omega}$ which is equinumerous to $A$ (the existence of such equinumerousness follows from the well-ordering theorem).

Proposition 20. $\boldsymbol{\Omega}$ is a strongly inaccessible class-cardinal, not a set cardinal.

Proof. Let $\mathbf{e}^{\left(\omega_{\nu}\right)}<\boldsymbol{\Omega}$, where $\omega_{\nu}$ is the initial ordinal number. Then the power-skand $\mathcal{P} \mathbf{e}^{\left(\omega_{\nu}\right)}$ is a set, and hence its permanent component is not in one-to-one correspondence with the permanent component $\boldsymbol{\Omega}_{0}$ of $\boldsymbol{\Omega}_{\left(0, \omega^{\kappa}\right)}, \kappa \geq 1$ is fixed; thus, $\mathcal{P} \mathbf{e}^{\left(\omega_{\nu}\right)}<\boldsymbol{\Omega}$. Moreover, for any $\mathbf{e}^{\left(\omega_{\alpha}\right)}<\boldsymbol{\Omega}$ and $\beta<\Omega$, the sum of cardinals, i.e., the initial ordinals, $\sum_{\alpha<\beta} \mathbf{e}^{\left(\omega_{\alpha}\right)}$ is a set and hence its permanent component is not in one-to-one correspondence with the permanent component $\boldsymbol{\Omega}_{0}$ of $\boldsymbol{\Omega}_{\left(0, \omega^{\kappa}\right)}, \kappa \geq 1$ is fixed and hence $\boldsymbol{\Omega}$ is not its ordinal type. 
Remark 15. The eschaton $\boldsymbol{\Omega}$ looks like the initial ordinal $\omega$ which is also a strongly inaccessible cardinal with respect to all finite numbers, and is the first transfinite ordinal. The same can be said for $\boldsymbol{\Omega}$, which is a strongly inaccessible class-cardinal with respect to all infinite numbers, i.e., all transfinite numbers, and is the first trans-infinite class-ordinal, or the first trans-definite ordinal, as it was called in [6].

Definition 11. By a proper generalized skand-class we understand $X_{\left(\alpha_{0}, \alpha\right)}$, at least one component $X_{\alpha^{\prime}}, \alpha_{0} \leq \alpha^{\prime}<\alpha, \alpha \in$ On of which is a proper class, in particular, a proper generalized self-similar skand-class.

Definition 12. By a hyper-skand and generalized hyper-skand we understand $X_{\left(\alpha_{0}, \boldsymbol{\Omega}\right)}$, whose components $X_{\alpha^{\prime}}, \alpha_{0} \leq \alpha^{\prime}<\Omega$, are elements of $\mathbf{V}[\mathcal{U}]$ and $\mathbf{V}[\mathcal{U}]^{(\Omega)}$, respectively, in particular, a self-similar hyper-skand and generalized self-similar hyper-skand $X_{\left(\alpha_{0}, \Omega\right)}(X)$.

Thus $\mathbf{O}_{(0, \omega)}=\{0,1,2, \ldots, \omega, \omega+1, \ldots,\{0,1,2, \ldots, \omega, \omega+1, \ldots,\{\ldots\}\}\}$,

$\mathbf{C}_{(0, \Omega)}=\{0,\{1,\{\ldots,\{n,\{\ldots\{\omega,\{\omega+1,\{\ldots\{\alpha,\{\ldots\}\}\}\}\}\}\}\}\}\}$ and

$\left.\left.\mathbf{E}_{(0, \boldsymbol{\Omega})}=\left\{\mathbf{e}^{(1)},\left\{\mathbf{e}^{(2)}, \ldots, \mathbf{e}^{(n)},\left\{\ldots\left\{\mathbf{e}^{(\omega)},\left\{\mathbf{e}^{(\omega+1)},\left\{\ldots\left\{\mathbf{e}^{(\alpha)},\{\ldots\}\right\}\right\}\right\}\right\}\right\}\right\}\right\}\right\}\right\}$, $\alpha \in \mathbf{O n}$, are examples of a skand-class, a hyper-skand and a generalized hyper-skand of all ordinals, respectively.

Definition 13. By a proper (generalized) hyper-skand-class we understand $X_{\left(\alpha_{0}, \Omega\right)}$, at least one component $X_{\alpha^{\prime}}, \alpha_{0} \leq \alpha^{\prime}<\Omega$ of which is a proper class, in particular, a proper (generalized) self-similar hyper-skandclass.

Remark 16. Definitions $11,12,13$ are very general in the sense that defined objects are outside of the $\mathbf{U}^{-}$-world and are not even subclasses of it. Nevertheless, there are operations similar to operations on proper classes which are subclasses of $\mathbf{V}[\mathcal{U}]^{(\Omega)}$. On the other hand, there are no such operations as the power-skand, singleton-skand or other set-theoretic operations. It is indeed true: "The content of a concept diminishes as its extension increases; if its extension becomes all-embracing, its content must vanish altogether". We need these definitions for descriptions of some aspects of the Skand Theory considered here.

\section{Applications to $\varepsilon$-numbers}

What we now want to show are applications of skands outside of the Skand Theory considered above.

Remark 17. The concept of a skand, i.e., objects $X_{\left(\alpha_{0}, \alpha\right)}$ above, is wider than its concrete realization as a system of embedded braces and the components thereof; it is not rigidly attached to curly brackets and it may also be a system of embedded round brackets, e.g., streams

$$
s=\left(a_{1},\left(a_{2},\left(a_{3},\left(\ldots .\left(a_{\omega},\left(a_{\omega+1},\left(\ldots\left(a_{\lambda},\left(a_{\lambda+1},(\ldots)\right)\right)\right)\right)\right)\right)\right)\right),\right.
$$


where $a_{\lambda} \in A, A$ is a set, $1 \leq \lambda<\Lambda \in \mathbf{O n}$, (see, in particular, the case of a countable system of embedded round brackets in [4], p. 34-35, 197-208), or a system of embedded angle brackets for the set theoretic operation modeling the operation of ordered pairs, etc. Skands $X_{(0, \alpha)}$ can be interpreted as a limit power or continued exponential with a basis which is an ordinal $\gamma_{0} \geq 1$ such that $X_{0}=\gamma_{0}$ is the first component of $X_{(0, \alpha)}$ and with the exponent $X_{(1, \alpha)}$, i.e.,

$$
X_{(0, \alpha)}=\gamma_{0}^{X_{(1, \alpha)}}
$$

such that $X_{\alpha^{\prime}}=\gamma_{\alpha^{\prime}}$ (notice that here we do not differ a singleton $\left\{\gamma_{\alpha^{\prime}}\right\}$ ) which is exactly $X_{\alpha^{\prime}}$ with its element itself $\gamma_{\alpha^{\prime}}$, the more so, as it is an individual), $\gamma_{\alpha^{\prime}} \in \mathbf{O n}, \gamma_{\alpha^{\prime}}>0,0 \leq \alpha^{\prime}<\alpha$, i.e., a transfinite sequence of basis-exponents

$$
X_{(0, \alpha)} \stackrel{\text { sign }}{=} \gamma_{0}^{\wedge} \gamma_{1}^{\wedge} \gamma_{2}^{\wedge} \ldots{ }^{\wedge} \gamma_{\omega}^{\wedge} \gamma_{\omega+1}^{\wedge} \ldots{ }^{\wedge} \gamma_{\alpha^{\prime}} \ldots \stackrel{\text { def }}{=} \gamma_{0}^{X_{(1, \alpha)}}=\ldots=\gamma_{0}^{\gamma_{1}^{\gamma_{2}}}
$$

Note only that the latter term in (39) should be properly defined (see below), and in (39) it is only a designation, nothing else.

We shall use such a skand-exponent in application to the theory of $\varepsilon$ numbers in the sense of Cantor. For convenience in the further designation of such a skand-exponent we prefer braces $\left\{\left\{\right.\right.$, i.e., $E_{(0, \alpha)}=\left\{\gamma_{0},\left\{\gamma_{1},\{\ldots\}\right\}\right\}$ to the power-sign ${ }^{\wedge}$ in (39) or expressions like $\left[\gamma_{0}, \gamma_{1}, \gamma_{2}, \ldots \gamma_{\omega}, \gamma_{\omega+1}, \ldots, \gamma_{\alpha^{\prime}}, \ldots\right]$ in [51], as a descending sequence of two-element non-well-founded sets $E_{(0, \alpha)} \ni E_{(1, \alpha)} \ni \ldots \ni E_{\left(\alpha^{\prime}, \alpha\right)} \ni \ldots$, where $E_{\left(\alpha^{\prime}, \alpha\right)}=\left\{\gamma_{\alpha^{\prime}}, E_{\left(\alpha^{\prime}+1, \alpha\right)}\right\}$, $0 \leq \alpha^{\prime}<\alpha$.

Let $\gamma, \xi$ be arbitrary ordinal numbers such that $\gamma>0$ and $\xi \geq 0$. We recall that the $\xi$ th power of $\gamma$, i.e., $\gamma^{\xi}$, is defined by the following transfinite induction:

$$
\begin{gathered}
\gamma^{0}=1, \\
\gamma^{\alpha+1}=\gamma^{\alpha} \gamma, \\
\gamma^{\lambda}=\lim _{\alpha<\lambda} \gamma^{\alpha},
\end{gathered}
$$

for limit-numbers $\lambda=\lim _{\alpha<\lambda} \alpha$.

And as in arithmetic, $\gamma$ is called the basis, $\xi$, the exponent, of the power $\gamma^{\xi}$.

Consider now the following equation:

$$
\gamma^{\xi}=\xi
$$


with indeterminate $\xi$.

The roots $\xi=\alpha$ of the equation (43) in the case $\gamma=\omega$ and $\alpha<\omega_{1}$, where $\omega_{1}$ is the smallest non-denumerable ordinal, Cantor called epsilonnumbers. More precisely, "to distinguish them from all other numbers I call them the " $\varepsilon$-numbers of the second number-class" ([16], §20).

We can omit these restrictions of Cantor's, since all his results on the $\varepsilon$-numbers are valid in general cases. Here we repeat Cantor's construction in a generalized form.

Let $\gamma>0$ be a fixed ordinal. If $\alpha>0$ is any ordinal number which does not satisfy the equation (43), it determines an increasing sequence $\alpha_{n}$, $0 \leq n<\omega$, by means of the equalities

$$
\alpha_{0}=\alpha, \quad \alpha_{1}=\gamma^{\alpha} \quad \alpha_{2}=\gamma^{\alpha_{1}}, \ldots, \quad \alpha_{n}=\gamma^{\alpha_{n-1}}, \ldots .
$$

Then $\lim \alpha_{n} \stackrel{\text { def }}{=} \sup \alpha_{n}=E(\alpha)$ of this increasing sequence always exists because $\mathbf{O n}$ is well-ordered, and we call it an $\varepsilon$-number, too.

Indeed, in the trivial case, when $\gamma=1$, the only root of the equation (43) is evidently $\xi=1$, and hence this actually increasing sequence in (44) is the constant sequence $\alpha_{n}=1,0 \leq n<\omega$, and thus $\lim _{n} \alpha_{n}=\sup _{n} \alpha_{n}=$ $E(\alpha)=1$, for every $\alpha>1$.

If $\gamma>1$, then (44) is an ascending sequence (in Cantor's terminology, an "ascending fundamental series") because

$$
\gamma>1 \Longrightarrow \gamma^{\alpha} \geq \alpha
$$

for every $\alpha \geq 0$ (see, e.g., [36], Chap. VII, $\S 6$ ); in our case, when $\alpha>1$ and does not satisfy the equation (43), we have $\gamma^{\alpha}>\alpha$ and, by (45), $\gamma^{\gamma^{\alpha}}>\gamma^{\alpha}$, and so on. Consequently, (44) is an ascending sequence; indeed, for all $0<n<\omega, \alpha_{1}>\alpha_{0}, \alpha_{2}>\alpha_{1}, \alpha_{3}>\alpha_{2}, \ldots, \alpha_{n}>\alpha_{n-1}, \ldots$.

Put now $E(\alpha)=\lim _{n} \alpha_{n}=\sup _{n} \alpha_{n}$ which is a limit-ordinal number and always exists, because for the set $A=\left\{\alpha_{0}, \alpha_{1}, \ldots, \alpha_{n}, \ldots\right\}$, there exists an ordinal $\beta<\beta_{0}$ (for some fixed $\beta_{0}$ ), which is greater than each element of $A$ (see [36], Chap. VII, $\S 2$, Theorem 6). Consequently, the least of such $\beta$, which always exists in the well-ordered set $\left(0, \beta_{0}\right)$ since each of its subsets has the smallest element, is the desired ordinal number $E(\alpha)$.

By (41), (42), the function $f(\alpha)=\gamma^{\alpha}$ is ascending and continuous; therefore, we have $\gamma^{E(\alpha)}=\gamma^{\lim _{n} \gamma^{\alpha_{n}}}=\lim _{n} \gamma^{\alpha_{n}}=\lim _{n} \alpha_{n+1}=E(\alpha)$; i.e., $E(\alpha)$ satisfies (43).

Cantor considered the case $\gamma=\omega, \alpha=1$ and proved that

$$
E(1)=\lim _{n} \omega_{n}
$$


where

$$
\alpha_{0}=1 \quad \alpha_{1}=\omega \quad \alpha_{2}=\omega^{\alpha_{1}}, \quad \ldots, \quad \alpha_{n}=\omega^{\alpha_{n-1}}, \quad \ldots,
$$

is an $\varepsilon$-number [16], $\S 20$, [Theorem] A. Moreover, $\varepsilon_{0}=E(1)=\lim _{n} \omega_{n}$ is the least of all the $\varepsilon$-numbers $([16], \S 20$, [Theorem] B). (This, of course, is true in his own sense; in our general construction there are two more $\varepsilon$-numbers 1 , when $\gamma=1$ and $\omega$, when $1<\gamma<\omega$ and $\alpha=\gamma$, which are evidently smaller than $\varepsilon_{0}$.)

He also showed that after the least $\varepsilon$-number, $\varepsilon_{0}$, there follows then the next greater one:

$$
\varepsilon_{1}=E\left(\varepsilon_{0}+1\right),
$$

and so on; i.e., there is the following formula of recursion:

$$
\varepsilon_{n}=E\left(\varepsilon_{n-1}+1\right),
$$

$1 \leq n<\omega .([16], \S 20,[$ Theorem] D).

The $\operatorname{limit} \lim \varepsilon_{\nu_{n}}$ of any ascending sequence $\varepsilon_{\nu_{0}}, \varepsilon_{\nu_{1}}, \ldots, \varepsilon_{\nu_{n}}, .$. of $\varepsilon$-number $\varepsilon_{\nu_{n}}, 0 \leq n<\omega$, is an $\varepsilon$-number, too. ([16], §20, [Theorem] E). Finally, all the totality of $\varepsilon$-numbers of the second number-class is a well-ordered set

$$
\varepsilon_{0}, \varepsilon_{1}, \ldots, \varepsilon_{n} \ldots \varepsilon_{\omega}, \varepsilon_{\omega+1}, \ldots \varepsilon_{\alpha^{\prime}}, \ldots
$$

of the second number-class type, and has thus the power $\aleph_{1}$, where $0 \leq$ $\alpha^{\prime}<\Omega$, ([16], §20, [Theorem] F); i.e., $\Omega$ is the initial ordinal, in recent terminology, which is the first after the initial ordinal $\omega$. (Here we quote notations $\omega_{n}, n \geq 1$, and $\Omega$ in [34], pp. 196, 199, literally; thus, do not confuse them with $\omega_{\kappa}, \kappa=1,2, \ldots$, and $\boldsymbol{\Omega}$ below, respectively, which denote absolutely different objects.)

We also mention two more of Cantor's results. If $\varepsilon^{\prime}$ is any $\varepsilon$-number, $\varepsilon^{\prime \prime}$ is the next greater $\varepsilon$-number, and $\alpha$ is any number which lies between them:

$$
\varepsilon^{\prime}<\alpha<\varepsilon^{\prime \prime}
$$

then $E(\alpha)=\varepsilon^{\prime \prime}([16], \S 20$, [Theorem] $\mathrm{C})$; and if $\varepsilon$ is any $\varepsilon$-number and $\alpha$ is any number such that $1<\alpha<\varepsilon$, then $\varepsilon$ satisfies the three equations:

$$
\alpha+\varepsilon=\varepsilon ; \alpha \varepsilon=\varepsilon ; \alpha^{\varepsilon}=\varepsilon
$$

([16], §20, [Theorem] G).

Note that for the $\varepsilon$-number $\omega$ in our general construction, for each $\alpha$ such that $1<\alpha<\omega,(52)$ also holds.

All these results of Cantor are valid in the general case of course, with natural corrections: ordinal types, the cardinality of the initial ordinals, etc. But we want more. We want with the help of self-similar skands to clarify this general situation. 
Cantor's formula (46) of the least (in Cantor's sense) $\varepsilon$-number $\varepsilon_{0}$ can be symbolically written in the following form:

$$
\varepsilon_{0}=\omega^{\omega^{\omega \cdot{ }^{\omega}}} \quad ;
$$

i.e., by misuse of language, " $\varepsilon_{0}$ is the power of $\omega$ whose exponent is the power of $\omega$, whose exponent is the power $\omega$, etc., more precisely, $\omega$ times 'the power of ", or "the first 'limit power' of $\omega$ whose exponents at each $n$th place of the skand-exponent $E_{(0, \omega)}$ is $\omega, 1 \leq n<\omega$ ". How else?

Let us denote (53) by a bit shorter formula:

$$
\varepsilon_{0}=\omega^{\omega^{\omega \cdot}}=E_{(0, \omega)}(\omega) \stackrel{\text { def }}{=} \omega^{E_{(1, \omega)}(\omega)},
$$

where $X_{(0, \omega)}(\omega)$ denotes the self-similar skand-exponent of length $\omega$ whose components $E_{n}, 0 \leq n<\omega$, are $\omega$ (see Remarks 1, 17). Actually, we want to generalize this notation and the notion of the "limit power" to the following one:

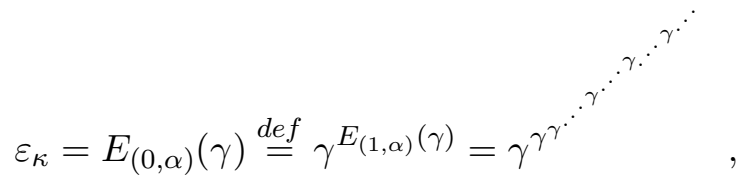

for $\gamma>1, \alpha=\omega^{\kappa}, \kappa \geq 1$, and $\kappa \in \mathbf{O}$.

Actually, we have to explain the meaning of the symbol $E_{(0, \alpha)}(\gamma)=$

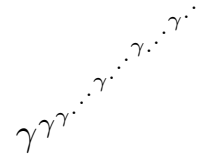

as a good way of describing all possible $\varepsilon$-numbers because, by Definition $2, E_{\left(0, \omega^{\kappa}\right)}(\gamma)=E_{\left(1, \omega^{\kappa}\right)}(\gamma)$ and therefore,

$$
\gamma^{E_{\left(0, \omega^{\kappa}\right)}(\gamma)}=\gamma^{E_{\left(1, \omega^{\kappa}\right)}(\gamma)}=E_{\left(0, \omega^{\kappa}\right)}(\gamma) ;
$$

i.e., $E_{\left(0, \omega^{\kappa}\right)}(\gamma)$ in $(55)$ is a root of the equation (43), for an arbitrary ordinal $\kappa \geq 1$, and hence is an $\varepsilon$-number.

Here is an explicit explanation of this idea.

Definition 14. By a skand-exponent $E_{\left(\alpha_{0}, \alpha\right)}$ of length $l=\alpha-\alpha_{0} \geq \omega$ we understand a system of embedded curly braces, indexed by $\alpha^{\prime} \in\left(\alpha_{0}, \alpha\right)$, all of whose components are one-element, moreover, for each $\alpha_{0} \leq \alpha^{\prime}<\alpha$, $E_{\alpha^{\prime}}=\left\{\gamma_{\alpha^{\prime}}\left\{\right.\right.$ or $E_{\alpha^{\prime}}=\left\{\gamma_{\alpha^{\prime}}\right\}$, if $\alpha^{\prime}=\alpha-1$, where $\gamma_{\alpha^{\prime}} \neq 0$ and $\gamma_{\alpha^{\prime}} \in$ On. If all components are equal to $\gamma$, then we write $E_{\left(\alpha_{0}, \alpha\right)}(\gamma)=\{\gamma,\{\gamma,\{\ldots\}\}\}$ and consiner it as a descending sequence of a two-elements non-well-founded set $E_{\left(\alpha_{0}, \alpha\right)} \ni\left\{\gamma, E_{\left(\alpha_{0}+1, \alpha\right)}\right\} \ni\left\{\ldots \ni\left\{\gamma, E_{\left(\alpha_{0}+\alpha^{\prime}, \alpha\right)}\right\} \ni \ldots, 0 \leq \alpha^{\prime}<\alpha-\alpha_{0}\right.$. 
Definition 15. Two skand-exponents $E_{\left(\alpha_{0}, \alpha\right)}^{1}$ and $E_{\left(\beta_{0}, \beta\right)}^{2}$ are called equal if the segments $\left(\alpha_{0}, \alpha\right)$ and $\left(\beta_{0}, \beta\right)$ are isomorphic as well-ordered sets, where $\varphi:\left(\alpha_{0}, \alpha\right) \rightarrow\left(\beta_{0}, \beta\right)$ is this isomorphism, and the corresponding components $E_{\alpha^{\prime}}^{1}$ and $E_{\beta^{\prime}}^{2}$ are equal, for each $\beta^{\prime}=\varphi\left(\alpha^{\prime}\right), \alpha_{0} \leq \alpha^{\prime}<\alpha$.

Definition 16. By an $\omega$-limit power of the skand-exponent

$$
E_{\left(\alpha_{0}, \omega\right)}=\left\{\gamma_{\alpha_{0}},\left\{\gamma_{\alpha_{0}+1},\{\ldots\}\right\}\right\}=\gamma_{\alpha_{0}}^{\gamma_{\alpha_{0}+1}^{*}}
$$

we understand 1 , if $\gamma_{\alpha_{0}}=1$; if $\gamma_{\alpha_{0}} \neq 1$, then we understand $\lim _{n} \beta_{\alpha_{0}+n}=$ $\sup _{n} \beta_{\alpha_{0}+n}$ of the following $\omega$-sequence

$$
E_{\alpha_{0}, \alpha_{0}+1}, E_{\left(\alpha_{0}, \alpha_{0}+2\right)}, \ldots E_{\left(\alpha_{0}, \alpha_{0}+n+1\right)}, \ldots
$$

where $E_{\left(\alpha_{0}, \alpha_{0}+1\right)}=\gamma_{\alpha_{0}}$ and

$$
E_{\left(\alpha_{0}, \alpha_{0}+n+1\right)}=\gamma_{\alpha_{0}}^{\gamma_{\alpha_{0}+1}^{\gamma_{\alpha_{0}+n-1}^{\gamma_{\alpha_{0}+n}}}},
$$

for each $1 \leq n<\omega$, is understood in the usual way: we descend, begin-

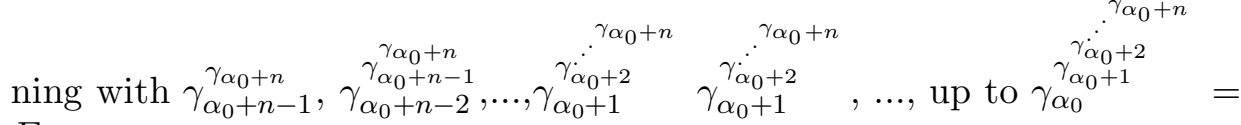
$E_{\left(\alpha_{0}, \alpha_{0}+n+1\right)}$.

Now, first of all, we shall prove the following lemmas.

Lemma 2. Let $\varepsilon_{0}$ and $\varepsilon_{1}$ be the first and the second $\varepsilon$-numbers in Cantor's sense. Then for each $\gamma, \varepsilon_{0} \leq \gamma<\varepsilon_{1}$, we have

$$
E_{(0, \omega)}(\gamma)=\varepsilon_{1}
$$

in particular,

$$
E_{(0, \omega)}\left(\varepsilon_{0}\right)=\varepsilon_{0}^{\varepsilon_{0}^{\varepsilon_{0}}}=\varepsilon_{1} .
$$

Moreover, for each $\omega$-sequence of ordinals $\gamma_{0}, \gamma_{1}, \ldots, \gamma_{n}, \ldots$ such that $\varepsilon_{0} \leq$ $\gamma_{n}<\varepsilon_{1}, 0 \leq n<\omega$, we have

$$
E_{(0, \omega)}\left\{\gamma_{0},\left\{\gamma_{1},\left\{\gamma_{2},\{\ldots\}\right\}\right\}\right\}=\gamma_{0}^{\gamma_{1}^{\gamma_{2}}}=\varepsilon_{1} .
$$

Proof. Since by the third equation in (52), $\gamma_{0}^{\varepsilon_{1}}=\varepsilon_{1}$, we have $\gamma_{0}^{\gamma_{1}}<$ $\gamma_{0}^{\varepsilon_{1}}=\varepsilon_{1}$ as well as $\gamma_{1}^{\gamma_{2}}<\gamma_{1}^{\varepsilon_{1}}=\varepsilon_{1}$, and hence $\gamma_{0}^{\gamma_{1}^{\gamma_{2}}}<\varepsilon_{1}$. The same argument says that $\gamma_{0}^{\gamma_{1}^{\gamma}}<\varepsilon_{1}$, for each $0 \leq n<\omega$. Consequently,

$$
E_{(0, \omega)}=\left\{\gamma_{0}\left\{\gamma_{1}\left\{\gamma_{1} \ldots\right\}\right\}\right\}=\gamma_{0}^{\gamma_{1}^{*}}=\lim _{n} E_{(0, n)}=\lim _{n} \gamma_{0}^{\gamma_{1}^{\gamma_{2}^{*}}} \leq \varepsilon_{1} .
$$


In particular, for $\gamma=\gamma_{n}, 0 \leq n<\omega, E_{(0, \omega)}(\gamma)=\gamma^{\gamma^{*}} \leq \varepsilon_{1}$.

In spite of the fact that $E_{(0, \omega)}(\gamma)$ satisfies the equation (43) and seems to be an $\varepsilon$-number, it is not a definition in Cantor's sense, and it might be something different from Cantor's classical $\varepsilon$-numbers and $X_{(0, \omega)}(\gamma)=$ $\gamma^{\gamma}<\varepsilon_{1}$. Why not? We shall show now that this is not the case. Clearly, by $\varepsilon_{0} \leq \gamma, E_{(0, \omega)}\left(\varepsilon_{0}\right) \leq E_{(0, \omega)}(\gamma)$ and, by $\varepsilon_{0} \leq \gamma_{n}, 0 \leq n<\omega, E_{(0, \omega)}\left(\varepsilon_{0}\right) \leq$ $E_{(0, \omega)}=\gamma_{0}^{\gamma_{1}^{\gamma_{2}}}$. On the other hand, by $\omega<\varepsilon_{0}$, we obtain

$$
E_{(0, n+1)}=\omega^{\omega \cdot \cdot^{\omega^{\varepsilon_{0}}}}<\varepsilon_{0}^{\varepsilon_{0}^{\cdot \varepsilon_{0}^{\varepsilon_{0}^{\varepsilon_{0}}}}}=X_{(0, n+1)}\left(\varepsilon_{0}\right) .
$$

Since $\varepsilon_{0}+1<\varepsilon_{0}^{\varepsilon_{0}}<\varepsilon_{1}$, by (48) and [Theorem] C, we obtain

$$
\begin{gathered}
\varepsilon_{1}=\lim _{n} E_{(0, n+1)}=\lim _{n} \omega^{\omega \cdot{ }^{\varepsilon_{0}^{\varepsilon_{0}}}} \leq \lim _{n} \varepsilon_{0}^{\varepsilon_{0} \cdot{ }^{\varepsilon_{0}^{\varepsilon_{0}}}}=\lim _{n} E_{(0, n+1)}\left(\varepsilon_{0}\right) \leq \\
\leq \lim _{n}\left\{\gamma_{0}\left\{\gamma_{1}\left\{\gamma_{2}\left\{\ldots\left\{\gamma_{n}\right\}\right\}\right\}\right\}\right\}=\gamma_{0}^{\gamma_{1}^{\gamma_{2}}} \leq \varepsilon_{1} .
\end{gathered}
$$

Consequently, $E_{(0, \omega)}=\gamma_{0}^{\gamma_{1}^{\gamma}}=\varepsilon_{1}$, which completes the proof of Lemma 2 .

Lemma 3. Let $\varepsilon^{\prime}$ and $\varepsilon^{\prime \prime}$ be neighboring $\varepsilon$-numbers in Cantor's sense. Then for each $\gamma, \varepsilon^{\prime} \leq \gamma<\varepsilon^{\prime \prime}$, we have

$$
E_{(0, \omega)}(\gamma)=\varepsilon^{\prime \prime}
$$

in particular,

$$
E_{(0, \omega)}\left(\varepsilon^{\prime}\right)=\varepsilon^{\prime \varepsilon^{\prime \varepsilon^{\prime} \cdot}}=\varepsilon^{\prime \prime}
$$

Moreover, for each $\omega$-sequence of ordinals $\gamma_{0}, \gamma_{1}, \ldots, \gamma_{n}, \ldots$ such that $\varepsilon^{\prime} \leq$ $\gamma_{n}<\varepsilon^{\prime \prime}, 0 \leq n<\omega$, we have

$$
E_{(0, \omega)}\left\{\gamma_{0},\left\{\gamma_{1}\left\{\gamma_{2}\{\ldots\}\right\}\right\}\right\}=\gamma_{0}^{\gamma_{1}^{\gamma}}=\varepsilon^{\prime \prime}
$$

The Proof is absolutely similar to the proof of Lemma 2 .

Lemma 4. Let $\varepsilon_{0}, \varepsilon_{1}, \varepsilon_{2}, \ldots$ be an ascending $\omega$-sequence of $\varepsilon$-numbers. Then $E_{(0, \omega)}=\varepsilon_{0}^{\varepsilon_{1}}=\lim _{n} \varepsilon_{n}$, and hence is an $\varepsilon$-number in Cantor's sense. 
Proof. Since $\varepsilon_{0}<\varepsilon_{1}<\varepsilon_{2}<\ldots$ we have, by $(52), E_{(0, n+1)}=\varepsilon_{0}^{\varepsilon_{1}^{\varepsilon_{2}^{\varepsilon^{\varepsilon_{n}}}}}=$ $\varepsilon_{n}$. Consequently, by Cantor's Theorem E, $E_{(0, \omega)}=\lim _{n} E_{(0, n+1)}=\lim _{n} \varepsilon_{n}$ is an $\varepsilon$-number.

Lemma 5. For any ordinal number $\gamma \geq \omega, E_{(0, \omega)}(\gamma)=\gamma^{\gamma^{\gamma^{*}}}$ is an $\varepsilon$-number in the sense of Cantor. Moreover, for each increasing $\omega$-sequence of ordinals $\gamma_{0}, \gamma_{1}, \ldots, \gamma_{n}, \ldots$ such that $\omega \leq \gamma_{0}, E_{(0, \omega)}=\gamma_{0}^{\gamma_{1}^{\gamma_{2}^{*}}}$ is an $\varepsilon$-number in Cantor's sense.

Proof. By (45), we have $\omega^{\gamma} \geq \gamma$ and hence $\gamma<E(\gamma)$. This is the well-known fact that for each ordinal $\gamma$, there is an $\varepsilon$-number greater than $\gamma$ ([56], p. 327). Let $\varepsilon^{\prime \prime}$ be the least of such $\varepsilon$-numbers. Take the preceeding $\varepsilon^{\prime}$ which always exists because $\varepsilon^{\prime \prime}$ cannot be a limit of $\varepsilon$-numbers, otherwise, $\varepsilon^{\prime \prime}$ could not be the smallest $\varepsilon$-number greater than $\gamma$. Obviously, $\varepsilon^{\prime} \leq \gamma<\varepsilon^{\prime \prime}$. Then we apply Lemma 3 and obtain $E_{(0, \omega)}(\gamma)=\varepsilon^{\prime \prime}$.

Let now $\gamma_{0}, \gamma_{1}, \ldots, \gamma_{n}, \ldots$ be an increasing $\omega$-sequence, i.e., $\gamma_{0} \leq \gamma_{1} \leq \ldots \leq$ $\gamma_{n} \leq \ldots$ If it is stable, i.e., $\gamma_{n_{0}}=\gamma_{n_{0}+1}=\gamma_{n_{0}+2}=\ldots$, we put $\gamma=\gamma_{n_{0}}$ and apply the first assertion of Lemma 5, i.e. $E_{\left(n_{0}, \omega\right)}=\gamma_{n_{0}}^{\gamma_{n_{0}+1}^{\gamma_{n_{0}+2}^{\prime}}}=\gamma^{\gamma^{\gamma}}=\varepsilon^{\prime \prime}$.

$$
\gamma_{n_{0}-1}^{\varepsilon^{\prime \prime}}
$$

Then, clearly, $E_{(0, \omega)}=\gamma_{0}^{\gamma_{1}^{\gamma}}=\varepsilon^{\prime \prime}$.

If it is not stable, then without loss of generality we can assume that $\gamma_{0}<\gamma_{1}<\ldots<\gamma_{n}<\ldots$. Suppose now that there are only finite $\varepsilon$-numbers between these $\varepsilon$-numbers, e.g., $\gamma_{0}$ and $\gamma_{n_{0}}$, and denote the greatest of them by $\varepsilon^{\prime}$. Then, clearly, $E_{\left(n_{0}, \omega\right)}=\varepsilon^{\prime \prime}$ and $E_{(0, \omega)}=\varepsilon^{\prime \prime}$ as in the previous case. Finally, suppose that there is a ascending $\omega$-sequence $\varepsilon_{\nu_{0}}<\varepsilon_{\nu_{1}}<\ldots$ with is cofinal to a ascending $\omega$-sequence $\gamma_{0}<\gamma_{1}<\ldots$. Then, evidently, $\varepsilon^{\prime \prime}=\lim _{n} \gamma_{n}=\lim _{n} \varepsilon_{n}$ and the proof that $E_{(0, \omega)}=\varepsilon^{\prime \prime}$ is similar to that for Lemma 4 .

Remark 18. It is not true that for an arbitrary $\omega$-sequence of ordinals $\gamma_{0}, \gamma_{1}, \ldots, \gamma_{n}, \ldots$ the limit power $E_{(0, \omega)}=\gamma_{0}^{\gamma^{\cdot} \cdot{ }^{\gamma_{n}^{*}}}$ is an $\varepsilon$-number.

Indeed, consider the following $\underset{2}{\omega}$-sequence $\omega, 2,2, \ldots$, then

$$
\omega^{2^{2 \cdot}}=\lim _{n} \omega^{2^{2} \cdot{ }^{2}}=\omega_{n} \lim ^{2 \cdot{ }^{\cdot}}=\omega^{\omega} \text {. And } \omega^{\omega} \text { is not an } \varepsilon \text {-number. }
$$

Indeed, $2^{\omega^{\omega}}=2^{\lim _{n} \omega^{n}}=\lim _{n} 2^{\omega^{n}}=\lim _{n} \omega^{\omega^{n-1}}=\omega^{\lim _{n} \omega^{n-1}}=\omega^{\omega^{\omega}}>\omega^{\omega}$. The moral is that there are many $\omega$-sequences of ordinals and corresponding skand-exponents whose limit-powers are equal to $\varepsilon$-numbers; on the other 
hand, there are also a lot of $\omega$-sequences of ordinals and the corresponding skand-exponents whose limit-powers are not $\varepsilon$-numbers at all.

Remark 18 allows us to give the following

Definition 17. By an $\varepsilon$-number we understand any ordinal number of the form

$$
\varepsilon=E_{(0, \omega)}(\gamma), \gamma \geq 1
$$

and also for an arbitrary set $\mathcal{E}$ of such $\varepsilon$-numbers in (69) its supremum, i.e.,

$$
\varepsilon^{\prime}=\sup _{\varepsilon \in \mathcal{E}} \mathcal{E}
$$

It is clear that all $\varepsilon$-numbers in Definition 17 are $\varepsilon$-numbers in the sense of Cantor except two numbers: 1 because $1^{1}=1$ and (43) holds, and $\omega$, because $2^{\omega}=\omega$ and (43) holds, too. Let us denote these first two $\varepsilon$-numbers by $\varepsilon_{0}$ and $\varepsilon_{1}$, respectively. Since $\varepsilon_{2}=E_{(0, \omega)}(\omega)$, by Lemma 2, we obtain that it is the least $\varepsilon$-number in the sense of Cantor; and all finite indexes of $\varepsilon$-numbers in the sense of Cantor are shifted by adding 2 .

Definition 18. Let $\alpha=\omega^{\kappa}, \kappa \geq 1$. Then by a limit-power with the basis $\gamma>1$ and the same exponents we understand the skand-exponent $E_{(0, \alpha)}(\gamma)$, given by the following transfinite recursion:

$$
\begin{aligned}
& E_{(0, \omega)}(\gamma)=\bar{\varepsilon}_{1}, \quad \kappa=1 \\
& E_{\left(0, \omega^{2}\right)}(\gamma)=E_{(0, \omega)}\left(\bar{\varepsilon}_{1}\right)=\bar{\varepsilon}_{2}, \quad \kappa=2 ; \\
& E_{\left(0, \omega^{n}\right)}(\gamma)=E_{(0, \omega)}\left(\bar{\varepsilon}_{n-1}\right)=\bar{\varepsilon}_{n}, \quad \kappa=n ; \\
& E_{\left(0, \omega^{\omega}\right)}(\gamma)=\sup _{n} E_{\left(0, \omega^{n}\right)}(\gamma)=\sup _{n} E_{(0, \omega)}\left(\bar{\varepsilon}_{n-1}\right)=\bar{\varepsilon}_{\omega}, \quad \kappa=\omega ;
\end{aligned}
$$

In the general case

$$
\begin{gathered}
E_{\left(0, \omega^{\kappa}\right)}(\gamma)=E_{(0, \omega)}\left(\bar{\varepsilon}_{\kappa-1}\right)=\bar{\varepsilon}_{\kappa}, \quad \kappa-1<\kappa ; \\
E_{\left(0, \omega^{\kappa}\right)}(\gamma)=\sup _{\lambda<\kappa} E_{\left(0, \omega^{\lambda}\right)}(\gamma)=\sup _{\lambda<\kappa} E_{\left(0, \omega^{\lambda}\right)}(\gamma)=\bar{\varepsilon}_{\kappa}, \quad \nexists \kappa-1 .
\end{gathered}
$$

In accordance with Remark 18 and Definition 18, we are going to describe in canonical form all $\varepsilon$-numbers.

Theorem 4. There is a one-to-one correspondence between all ordinal numbers $\omega^{\kappa}, 0 \leq \kappa \in \mathbf{O n}$ and all $\varepsilon$-numbers $\varepsilon_{\kappa}$, defined in Definition 17, as follows: $\varepsilon_{0}=E_{(0,1)}(1)$ and $\varepsilon_{\kappa}=E_{\left(0, \omega^{\kappa}\right)}(2), \kappa \geq 1$.

Proof. If $\kappa=0$, we put $\varepsilon_{0}=1$. If $\kappa>0$, putting in Definition 18 $\gamma=2, \bar{\varepsilon}_{\kappa}=\varepsilon_{\kappa}$, we obtain, by Lemmas $2,3,4,5$, a successive enumeration of all $\varepsilon$-numbers in the sense of Definition 17 and thus all $\varepsilon$-numbers in the sense of Cantor. 
Theorem 4'. Put $\varepsilon_{0}=1$ and $\varepsilon_{1}=\omega$. Then for $\kappa>1$ one has the following formulas for all $\varepsilon$-numbers: $\varepsilon_{\kappa+1}=E_{(0, \omega)}(\varepsilon \kappa)$ and $\varepsilon_{\kappa+\omega}=$ $\lim _{0 \leq n<\omega} E_{(0, \omega)}\left(\varepsilon_{\kappa+n}\right)$.

Proof is inside the the proof of Theorem 4.

It is easy to see that if $\alpha \neq \omega^{\kappa}, \kappa>0$, then $E_{(0, \alpha)}(2)$ is not an $\varepsilon$-number. Indeed, put $\alpha=\omega+1$, then $E_{(0, \omega+1)}=E_{(1, \omega+1)}=\omega^{2}$; but $2^{\omega^{2}}=\omega^{\omega} \neq \omega^{2}$.

Corollary 6. The set $E_{(\alpha)}=\left\{\varepsilon_{\kappa} \mid \varepsilon_{\kappa}<\omega_{\alpha}\right\}$, i.e., the set of all $\varepsilon$-numbers $\varepsilon_{\kappa}$ such that $\varepsilon_{\kappa}$ less than the initial number $\omega_{\alpha}$ has a power greater than or equal to $\omega_{\alpha}$.

Proof. If the power of $E_{(\alpha)}$ were less than the power of $\omega_{\alpha}$, then $\varepsilon=$ $\sup \varepsilon_{\kappa}$ would be greater than all of the elements in $E_{(\alpha)}$, and it would $\varepsilon_{\kappa} \in E_{(\alpha)}$

be an $\varepsilon$-number whose power would be less than $\omega_{\alpha}$, because the latter is not the limit of a transfinite sequence of smaller powers. Thus $E_{(\alpha)} \cup\{\varepsilon\}$ would be larger than $E_{(\alpha)}$, which is in contradiction with the maximality of $E_{(\alpha)}$. Consequently, $\left|E_{(\alpha)}\right| \geq\left|\omega_{\alpha}\right|\left(\left|E_{(\alpha)}\right|\right.$ and $\left|\omega_{\alpha}\right|$ mean the cardinality of $E_{(\alpha)}$ and $\omega_{\alpha}$, respectively).

Corollary 7. The set $E_{(\alpha)}=\left\{\varepsilon_{\kappa} \mid \varepsilon_{\kappa}<\omega_{\alpha}\right\}$, i.e., the set of all $\varepsilon$ numbers $\varepsilon_{\kappa}$ such that $\varepsilon_{\kappa}$ less than the initial number $\omega_{\alpha}$ is a well-ordered set of the ordinal type $\omega_{\alpha}$ and thus has the power of $\omega_{\alpha}$.

Proof. For each $\kappa<\omega_{\alpha}$, clearly, $\varepsilon_{\kappa}<\omega_{\alpha}$. And this is a one-to-one correspondence between the set $\left(0, \omega_{\alpha}\right)=\left\{\kappa \mid \kappa<\omega_{\alpha}\right\}$ and the set $E_{(\alpha)}$. Moreover, if $\kappa^{\prime}<\kappa^{\prime \prime}<\omega_{\alpha}$, then $\varepsilon_{\kappa^{\prime}}<\varepsilon_{\kappa^{\prime \prime}}$, i.e., the ordinal type $\left(0, \omega_{\alpha}\right)$ is the same as $E_{(\alpha)}$. Since by Corollary $6,\left|E_{(\alpha)}\right| \geq\left|\omega_{\alpha}\right|$, we obtain that $\left|E_{(\alpha)}\right|=\left|\omega_{\alpha}\right|$.

Corollary 8. Every initial number $\omega_{\alpha}$ is an $\varepsilon$-number.

Proof. Clearly, $\omega_{\alpha}=\lim _{\varepsilon_{\kappa} \in E_{(\alpha)}} \varepsilon_{\kappa}$ and thus, by Definition 17, it is an $\varepsilon$-number.

Remark 19. The assertion of Corollary 8 was given in [56] and proved in the particular case $\omega_{1}$. It is well-known that each initial number $\omega_{\alpha}$ has a form $\omega_{\alpha}=\omega^{\lambda}$ for some ordinal $\lambda$ (see, e.g., [36], Chap. VIII, §3, Theorem 9 ), but usually no one points out that $\lambda=\omega_{\alpha}$.

Corollary 9. The well-ordered proper class of all $\varepsilon$-numbers is isomorphic to On.

The Proof is similar to that for Corollary 7 .

Remark 20. The limit power $E_{\left(0, \omega^{\kappa}\right)}(\gamma), \kappa \geq 1$, in Definition 18 should be better called the quantified power, because actually we quantify exponents by $\omega$-sequences of the same exponents: $1<\gamma<\omega$, then $\omega$, then $\varepsilon_{2}$, $\varepsilon_{3}, \ldots, \varepsilon_{\omega}, \ldots$, just to simplify the algorithm of transfinite recursion. We could do the same thing by a continued exponentials process, e.g., $E_{\left(0, \omega_{1}\right)}(2)$ : 


$$
\begin{aligned}
& E_{(0,2)}(2)=2^{2}, \ldots, E_{(0, n)}(2)=2^{2 \cdot{ }^{\cdot 2}}, \ldots E_{(0, \omega)}(2)=2^{2 \cdot}=\omega=\varepsilon_{1} ; \\
& E_{(0, \omega+1)}(2)=\omega^{2}, E_{(0, \omega+2)}(2)=\omega^{2^{2}}, \ldots E_{(0, \omega 2)}(2)=\omega^{2^{2}}=\omega^{\omega}=\varepsilon_{1}^{\varepsilon_{1}} ; \\
& E_{(0, \omega 2+1)}(2)=\omega^{\omega^{2}}, E_{(0, \omega+2)}(2)=\omega^{\omega^{2^{2}}}, \ldots E_{(0, \omega 3)}(2)=\omega^{\omega^{2^{2}}}=\omega^{\omega^{\omega}}, \ldots, \\
& E_{(0, \omega n)}(2)=\omega^{\omega \cdot} \quad, \ldots E_{\left(0, \omega^{2}\right)}(2)=\omega^{\omega^{\omega \omega}}=\varepsilon_{1}^{\varepsilon_{1}^{\varepsilon_{1}^{\varepsilon_{1}}}}=\varepsilon_{2} ; \\
& E_{\left(0, \omega^{2}+1\right)}(2)=\varepsilon_{2}^{2}, E_{\left(0, \omega^{2}+2\right)}(2)=\varepsilon_{2}^{2^{2}}, \ldots, E_{\left(0, \omega^{2}+\omega\right)}(2)=\varepsilon_{1}^{2^{2}}=\varepsilon_{2}^{\omega} \text {, } \\
& E_{\left(0, \omega^{2}+\omega+1\right)}(2)=\varepsilon_{2}^{\omega^{2}}, E_{\left(0, \omega^{2}+\omega+2\right)}(2)=\varepsilon_{2}^{\omega^{2}}, \ldots E_{\left(0, \omega^{2} 2\right)}(2)=\varepsilon_{2}^{\varepsilon_{2}} ; \\
& E_{\left(0, \omega^{3}\right)}(2)=\varepsilon_{2}^{\varepsilon_{2}^{\varepsilon_{2}}}=\varepsilon_{3} \\
& E_{\left(0, \omega^{\omega}\right)}(2)=\varepsilon_{1}^{\varepsilon_{2}^{\varepsilon_{3}^{\varepsilon_{4}}}}=\varepsilon_{\omega} \\
& \text {............................ } \\
& E_{\left(0, \omega_{1}\right)}(2)=\varepsilon_{1}^{\varepsilon_{\varepsilon_{2}}^{\varepsilon_{\omega}^{\varepsilon_{\omega}}}}=2^{E_{\left(1, \omega_{1}\right)}(2)}=2^{\omega_{1}}=\omega_{1} \text {, where } 1 \leq \alpha<\omega_{1} .
\end{aligned}
$$

There is a similar process in the case $\alpha=\omega_{\lambda}, \lambda \geq 2$, i.e., in the calculation of $E_{\left(0, \omega_{\lambda}\right)}$.

Proposition 21. For an arbitrary ordinal number $\alpha>0$ and any $1<\gamma<\omega, E_{(0, \alpha)}(\gamma)$ can be expressed by the following unique formula:

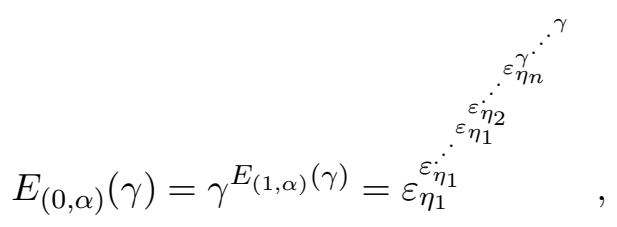

where $\varepsilon_{\eta_{1}}>\varepsilon_{\eta_{2}}>\ldots>\varepsilon_{\eta_{n}}$ are $\varepsilon$-numbers, $\eta_{1}>\eta_{2}>\ldots>\eta_{n}>0$ are ordinal numbers, and the quantization of them, and of $\gamma$ in the exponents is given by $\beta_{1}, \beta_{2}, \ldots, \beta_{n}, \beta_{n+1}$, respectively, $0 \leq \beta_{i}<\omega, i=1,2, \ldots, n, n+1$.

Proof. By Cantor's normal form of $\alpha$, it may be represented uniquely as

$$
\alpha=\omega^{\eta_{1}} \beta_{1}+\omega^{\eta_{2}} \beta_{2}+\ldots+\omega^{\eta_{n}} \beta_{n}+\beta_{n+1},
$$

where $\eta_{1}, \eta_{2}, \ldots, \eta_{n}$ is a descending sequence of ordinal numbers $>0$ and natural numbers $\beta_{1}, \beta_{2}, \ldots, \beta_{n}, \beta_{n+1}$ are $\geq 0$ (see, e.g., [36], Chap. VII, $\S 7$, Theorem 2). Then, by Theorem 4 and Remark 20, we obtain (77). 
Proposition 22. For every ordinal number $\gamma \geq 2$, the hyper-skand $E_{(0, \Omega)}(\gamma)$ is the greatest $\varepsilon$-class-number, i.e.,

$$
E_{(0, \boldsymbol{\Omega})}(\gamma)=\gamma^{E_{(1, \boldsymbol{\Omega})}(\gamma)}=\lim _{\alpha \in \mathbf{O n}} \varepsilon_{\alpha}=\varepsilon_{\boldsymbol{\Omega}}=\boldsymbol{\Omega}=\gamma^{\boldsymbol{\Omega}} .
$$

Proof. It is clear that for each ordinal number $\alpha>0, E_{(\alpha, \boldsymbol{\Omega})}(\gamma)$ is equal to $E_{(0, \boldsymbol{\Omega})}(\gamma)$. In other words, the skand $E_{(0, \boldsymbol{\Omega})}(\gamma)$ is self-similar, because each remainder $(\alpha, \boldsymbol{\Omega})$ as an ordered class is equal to $(0, \boldsymbol{\Omega})$, i.e., $(\alpha, \boldsymbol{\Omega})$ and $(0, \boldsymbol{\Omega})$ are isomorphic as ordered classes, in particular, for $\alpha=1$. Since, by Corollary 9 , the class of all $\varepsilon$-numbers has of the same ordinal type as $\boldsymbol{\Omega}$ we obtain that $\lim _{\alpha \in \mathbf{O} \mathbf{n}} \varepsilon_{\alpha}=\lim _{\alpha \in \mathbf{O} \mathbf{n}} \alpha=\boldsymbol{\Omega}$ and consequently, $\gamma^{\boldsymbol{\Omega}}=\gamma^{\lim _{\mathbf{O} \mathbf{n}} \varepsilon_{\alpha}}=$ $\lim _{\alpha \in \mathbf{O n}} \gamma^{\varepsilon_{\alpha}}=\lim _{\alpha \in \mathbf{O n}} \varepsilon_{\alpha}=\boldsymbol{\Omega}$, i.e., $\boldsymbol{\Omega}$ satisfies (43), and thus $\boldsymbol{\Omega}$ is an $\varepsilon$-classnumber.

\section{Applications to generalized real fractions}

Likewise, we denote here a transfinite $\alpha$-sequence $x_{\alpha_{0}}, x_{\alpha_{0}+1}, \ldots, x_{\alpha^{\prime}}, \ldots$, where $\alpha_{0} \leq \alpha^{\prime}<\alpha<\Omega$ and $x_{\alpha^{\prime}} \in \mathbf{U}^{\Omega}$, as a special skand

$$
X_{\left(\alpha_{0}, \alpha\right)}=\left\{x_{\alpha_{0}},\left\{x_{\alpha_{0}+1},\left\{\ldots\left\{x_{\alpha^{\prime}},\{\ldots\}\right\}\right\}\right\}\right\}
$$

as a descending sequence of two-elements sets $X_{\left(\alpha_{0}, \alpha\right)} \ni X_{\left(\alpha_{1}, \alpha\right)} \ni \ldots \ni$ $X_{\left(\alpha^{\prime}, \alpha\right)} \ni \ldots$, where $X_{\left(\alpha^{\prime}, \alpha\right)}=\left\{x_{\alpha^{\prime}}, X_{\left(\alpha^{\prime}+1, \alpha\right)}\right\}, \alpha_{0} \leq \alpha^{\prime}<\alpha$.

Let $\alpha$ be an ordinal of the 2 nd type, i.e. that having no predecessor; in particular, 0 is an ordinal of the 2nd type; its form $\alpha=\omega \nu$, where $\nu \geq 0$, is known. Recall also that ordinals of the 1st type are those having predecessors. (We have already used other terminology above as a limit ordinal number and an ordinal number which is not a limit number; an ordinal of the 2nd type and of the 1st type are shorter, and we now prefer the latter terminology.)

Consider now for a fixed $\alpha=\omega \nu, \nu \geq 1$, the set $\mathbf{A}_{\alpha}$ of all special skands (henceforth, in short: skands) $X_{(0, \alpha)}$ whose components $X_{\alpha^{\prime}}=0$ or $X_{\alpha^{\prime}}=1$ and as above we do not differ individuals 0 and 1 with their singletons $\{0\}$ and $\{1\}$ as it demands the definition of $X_{\alpha^{\prime}}$ : we simplify a notation without losting the sense of matter.

We endow $\mathbf{A}_{\alpha}$ with the following lexicographic linear ordering: $X_{(0, \alpha)}<$ $Y_{(0, \alpha)}$ iff there is an $\alpha^{\prime}, 0 \leq \alpha^{\prime}<\alpha$, such that $X_{\alpha^{\prime}}=0$ and $Y_{\alpha^{\prime}}=1$ and at whichever $\beta$ th place, $\beta<\alpha^{\prime}$, the elements are equal; i.e., $X_{\beta}=Y_{\beta}$. If in addition $X_{\beta}=1$ and $Y_{\beta}=0$, for all $\beta>\alpha^{\prime}$, then for such pairs only, there are no skands $Z_{(0, \alpha)}$ in $A_{\alpha}$ with $X_{(0, \alpha)}<Z_{(0, \alpha)}<Y_{(0, \alpha)}$. We call those pairs of neighboring skands twins. We shall identify them and denote the obtained new element in the canonical form, i.e., of a greater $Y_{(0, \alpha)}$, not 
forgetting that there is a different form of it, i.e., of a smaller $X_{(0, \alpha)}$, and using it when it is convenient.

Definition 18. By $\left.R_{\alpha}\right|_{[0,1]}=\left[0_{(0, \alpha)}, 1_{(0, \alpha)}\right]$ we denote the quotient set $\mathbf{A}_{\alpha} / \sim$ of $\mathbf{A}_{\alpha}$ ( identifies each pair of twins as one element) with the quotient linear ordering and call it a generalized real number (more precisely, fractional) unit interval of the power $2^{|\alpha|}$.

Here $0_{(0, \alpha)}$ and $1_{(0, \alpha)}$ are minimal and maximal elements (integers) of $\left.R_{\alpha}\right|_{[0,1]}$, i.e., skands with 0 and 1 at all places, respectively.

Definition 19. By $\left.Q_{\alpha}\right|_{[0,1]}$ we denote the subset of $\left.R_{\alpha}\right|_{[0,1]}$ of all skands $X_{(0, \alpha)}$ which are eventually 0 or 1 .

In particular, we distinguish in $\left.Q_{\alpha}\right|_{[0,1]}$ dyadic fractions, i.e. $\frac{1}{2^{\alpha^{\prime}}}$ as $X_{(0, \alpha)}$ such that $X_{\alpha^{\prime}-1}=1$ and $X_{\beta}=0$ for all $\beta \neq \alpha^{\prime}-1$, for each ordinal number $\alpha^{\prime}$ of the 1st kind, $1 \leq \alpha^{\prime}<\alpha$, (which is a twin to $Y_{(0, \alpha)}$ with $Y_{\beta}=0$ for $0 \leq \beta<\alpha^{\prime}$, and $Y_{\beta}=1$, for $\left.\alpha^{\prime} \leq \beta<\alpha\right)$ and also $\frac{1}{2^{\alpha^{\prime}}}$ as $X_{(0, \alpha)}$ such that $X_{\beta}=0$, for $0 \leq \beta<\alpha^{\prime}$, and $X_{\beta}=1$ for all $\beta \geq \alpha^{\prime}$, for each ordinal number $\alpha^{\prime}$ of the 2 nd kind, $0 \leq \alpha^{\prime}<\alpha$. In other words, in short, $\frac{1}{2^{\alpha^{\prime}}}$ are skands $X_{(0, \alpha)}$ which are eventually 1 .

Proposition 23. $\left.R_{\alpha}\right|_{[0,1]}$ and $\left.Q_{\alpha}\right|_{[0,1]}$ are the dense linear orderings and $\left.Q_{\alpha}\right|_{[0,1]}$ is dense in $\left.R_{\alpha}\right|_{[0,1]}$.

The Proof is an immediate consequence of Definitions 18 and 19 together with the definition of the ordering on $\mathbf{A}_{\alpha} / \sim$.

Theorem 5. The space $\left.R_{\alpha}\right|_{[0,1]}$ is continuous: i.e., every non-empty subset $S$ of $\left.R_{\alpha}\right|_{[0,1]}$ has a smallest upper bound $M_{(0, \alpha)}=\sup S$ and a greatest lower bound $m_{(0, \alpha)}=\inf S$ in $\left.R_{\alpha}\right|_{[0,1]}$.

Proof. If there exists a maximal element $\max S$ in $S$, then $\sup S=$ $\max S$, if there exists a minimal element $\min S$ in $S$, then $\inf S=\min S$.

Consider now the case when $S$ has no maximal element and prove that there exists $M_{(0, \alpha)}=\sup S$ in $\left.R_{\alpha}\right|_{[0,1]}$, i.e., $\left.M_{(0, \alpha)} \in R_{\alpha}\right|_{[0,1]}$ such that for all $X_{(0, \alpha)} \in S$ we have $X_{(0, \alpha)}<M_{(0, \alpha)}$ and for each $\left.Y_{(0, \alpha)} \in R_{\alpha}\right|_{[0,1]}$ such that $Y_{(0, \alpha)}<M_{(0, \alpha)}$ there is $Z_{(0, \alpha)} \in S$ such that $Y_{(0, \alpha)}<Z_{(0, \alpha)}$.

Indeed, there exists the smallest ordinal $\alpha_{1} \geq 0$ such that there is an element $X_{(0, \alpha)}^{1} \in S$ with $X_{\alpha_{1}}^{1}=1$ and for every $X_{(0, \alpha)} \in S, X_{\beta}=0$, for each $0=\alpha_{0} \leq \beta<\alpha_{1}$ (if $\alpha_{1}=0$, then conditions $X_{\beta}=0, \beta<\alpha_{1}$, are absent). Otherwise, $S$ should be $\left\{0_{(0, \alpha)}\right\}$ or the empty set $\emptyset$, which is impossible by assumption.

We shall define $\left.M_{(0, \alpha)} \in R_{\alpha}\right|_{[0,1]}$ by induction on its non-trivial components, putting at the beginning $M_{\alpha_{1}}=1$ and $M_{\beta}=0$, for each $0 \leq \beta<\alpha_{1}$, and then define the following subset $S^{1}=S \backslash\left\{X_{(0, \alpha)} \mid X_{(0, \alpha)} \in S, X_{(0, \alpha)}<\right.$ $\left.X_{(0, \alpha)}^{1}\right\}$ of $S=S^{0}$. 
Since $S$ has no maximal element and $X_{(0, \alpha)}^{1} \in S$, there exists the smallest ordinal $\alpha_{2}>\alpha_{1}$ such that there is an element $X_{(0, \alpha)}^{2}>X_{(0, \alpha)}^{1}$ in $S^{1}$ with $X_{\alpha_{2}}^{2}=1$, and for every $X_{(0, \alpha)} \in S^{1}, X_{\beta}=0$, for each $\alpha_{1}<\beta<\alpha_{2}$.

We continue to define $M_{(0, \alpha)}$ for the next series of indexes by putting $M_{\alpha_{2}}=1$ and $M_{\beta}=0$, for each $\alpha_{1}<\beta<\alpha_{2}$, and define now $S^{2}=$ $S^{1} \backslash\left\{X_{(0, \alpha)} \mid X_{(0, \alpha)} \in S^{1}, X_{(0, \alpha)}<X_{(0, \alpha)}^{2}\right\}$.

Suppose that, for each $1 \leq k \leq n<\omega$, we have already found the smallest ordinal $\alpha_{k}>\alpha_{k-1}$ and elements $X_{(0, \alpha)}^{k}>X_{(0, \alpha)}^{k-1}$ such that $X_{\alpha_{k}}^{k}=1$ and in addition for every $X_{(0, \alpha)} \in S^{k-1}$ its components $X_{\beta}=0$, where $\alpha_{k-1}<\beta<\alpha_{k}$. Suppose also that we have already defined the next components of $M_{(0, \alpha)}$, by putting $M_{\alpha_{k}}=1$ and $M_{\beta}=0$, for each $\alpha_{k-1}<$ $\beta<\alpha_{k}$, as well as the set $S^{k}=S^{k-1} \backslash\left\{X_{(0, \alpha)} \mid X_{(0, \alpha)} \in S^{k-1}, X_{(0, \alpha)}<\right.$ $\left.X_{(0, \alpha)}^{k}\right\}$. Notice that in this induction we put formally $X_{(0, \alpha)}^{0}=0_{(0, \alpha)}$.

Since $S^{n}$ has no maximal element and $X_{(0, \alpha)}^{n} \in S$, there exists the smallest ordinal $\alpha_{n+1}>\alpha_{n}$ such that there is an element $X_{(0, \alpha)}^{n+1}>X_{(0, \alpha)}^{n}$ in $S^{n}$ with $X_{\alpha_{n+1}}^{n+1}=1$ and for every $X_{(0, \alpha)} \in S^{n}, X_{\beta}=0$, for each $\alpha_{n}<\beta<\alpha_{n+1}$. We put $M_{\alpha_{n+1}}=1$ and $M_{\beta}=0$, for each $\alpha_{n}<\beta<\alpha_{n+1}$, and define $S^{n+1}=S^{n} \backslash\left\{X_{(0, \alpha)} \mid X_{(0, \alpha)} \in S^{n}, X_{(0, \alpha)}<X_{(0, \alpha)}^{n+1}\right\}$.

Thus $S^{n}$ are defined for all $0 \leq n<\omega$ and we can consider their intersection $\bigcap_{n} S^{n}$. If $\bigcap_{n} S^{n}=\emptyset$, i.e., there are no more elements $X_{(0, \alpha)}$ in $S$ with $X_{\beta}{ }^{n}=1, \beta>{ }^{n} \alpha_{n}$, for each $0 \leq n<\omega$, then we put $M_{\beta}=0$, for all $\alpha_{\omega} \leq \beta<\alpha$, where $\alpha_{\omega}=\lim _{n} \alpha_{n}$. Since for each $0 \leq \alpha^{\prime}<\alpha, M_{\alpha^{\prime}}$ has been already defined, we obtain an element $\left.M_{(0, \alpha)} \in R_{\alpha}\right|_{[0,1]}$ and we claim that it is sup $S$. Indeed, by construction, for every $X_{(0, \alpha)} \in S$, we have $X_{(0, \alpha)}<M_{(0, \alpha)}$. If $\left.Y_{(0, \alpha)} \in R_{\alpha}\right|_{[0,1]}$ and $Y_{(0, \alpha)}<M_{(0, \alpha)}$, then there is a minimal index $\alpha_{n}, 0 \leq n<\omega$, such that $Y_{\alpha_{n}}=0$ and $M_{\alpha_{n}}=1$. Take $X_{(0, \alpha)}^{n} \in S$. It is clear that $Y_{(0, \alpha)}<X_{(0, \alpha)}^{n}$. Thus in this case the existence of sup $S$ is proved.

If $\bigcap_{n} S^{n} \neq \emptyset$, then we define $S^{\omega}=\bigcap_{n} S^{n}$. Since $S^{\omega} \neq \emptyset$ there exists the smallest ordinal $\alpha_{\omega+1} \geq \alpha_{\omega}$ such that there is an element $X_{(0, \alpha)}^{\omega+1}$ in $S^{\omega}$ with $X_{(0, \alpha)}^{\omega+1}>X_{(0, \alpha)}^{n}$, for each $0 \leq n<\omega, X_{\alpha_{\omega+1}}^{\omega+1}=1$, and for every $X_{(0, \alpha)} \in$ $S^{\omega}, X_{\beta}=0$, for each $\alpha_{\omega} \leq \beta<\alpha_{\omega+1}$ (if $\alpha_{\omega+1}=\alpha_{\omega}$, then conditions $X_{\beta}=0, \beta<\alpha_{\omega+1}$, are absent). We put $M_{\alpha_{\omega+1}}=1$ and $M_{\beta}=0$, for each $\alpha_{\omega} \leq \beta<\alpha_{\omega+1}$ and define the following set $S^{\omega+1}=S^{\omega} \backslash\left\{X_{(0, \alpha)} \mid X_{(0, \alpha)} \in\right.$ $\left.S^{\omega}, X_{(0, \alpha)}<X_{(0, \alpha)}^{\omega+1}\right\}$. Then we continue our algorithm as above.

Since each step of our inductive construction enlarges the index $\alpha_{\nu}$, $1 \leq \nu$, at least by 1 , we shall exhaust all of $0 \leq \alpha^{\prime}<\alpha$ and obtain an 
element $\left.M_{(0, \alpha)} \in R_{\alpha}\right|_{[0,1]}$ such that $X_{(0, \alpha)}^{\nu}<M_{(0, \alpha)}$, for each $\nu \geq 1$. Since $\bigcap_{\nu} S^{\nu}=\emptyset$ (otherwise, $M_{(0, \alpha)}$ should be an elements of $\bigcap_{\nu} S^{\nu}$ and thus the greatest element of $S$ ) we conclude that $M_{(0, \alpha)}=\sup S$. Indeed, for each $X_{(0, \alpha)} \in S, X_{(0, \alpha)}<M_{(0, \alpha)}$ and if $\left.Y_{(0, \alpha)} \in R_{\alpha}\right|_{[0,1]}$ and $Y_{(0, \alpha)}<M_{(0, \alpha)}$, then there is the smallest ordinal $\alpha_{\nu}$ condidered above such that $Y_{\alpha_{\nu}}=0$ and $M_{\alpha_{\nu}}=1$. Take $X_{(0, \alpha)}^{\nu} \in S$ and by construction $Y_{(0, \alpha)}<X_{(0, \alpha)}$.

The proof of the existence of inf $S$ in the case when $S$ has no minimal element is absolutely similar. We omit details, but there is another proof of it. Putting $S^{*}=\left\{1_{(0, \alpha)}-X_{(0, \alpha)} \mid X_{(0, \alpha)} \in S\right\}$, we obtain $\left.S^{*} \subset R_{\alpha}\right|_{[0,1]}$ and $S^{*}$ has no maximal element. By the above proof, there exists a smallest upper bound $M_{(0, \alpha)}$ of $S^{*}$. If we put $m_{(0, \alpha)}=1_{(0, \alpha)}-M_{(0, \alpha)}$, then it is a greatest lower bound of $S$. (For the meaning of $1_{(0, \alpha)}-X_{(0, \alpha)}$ see the next paragraph.)

Theorem 6. The covering dimension of a topological space $\left.R_{\alpha}\right|_{[0,1]}$ in the order topology is equal to 1 , i.e., $\left.\operatorname{dim} R_{\alpha}\right|_{[0,1]}=1$.

Proof. It is well known that every linearly ordered space $X$ is hereditarily normal ([11] Bourbaki, [1948]).

For every normal space $X \operatorname{dim} X \leq \operatorname{Ind} X$ ([57] Vedenissoff, [1939]).

For every space $Y$ the properties $\operatorname{dim} Y=0$ and $\operatorname{Ind} Y=0$ are equivalent and have as their consequence the normality of $Y$ ([2] Chap. II, §3, Proposition 3, p. 170).

It is also known that if every hereditarily normal space $X$ is a union of two spaces $Y$ and $Z$ such that $\operatorname{Ind} Y=0$ and $\operatorname{Ind} Z=0$, then Ind $X \leq 1$ ([35] Katětov, [1951]).

Putting $Y=\left.Q_{\alpha}\right|_{[0,1]}$ and $Z=\left.R_{\alpha}\right|_{[0,1]} \backslash Y$, we notice that since $Y$ and $Z$ are dense in themselves and in $\left.R_{\alpha}\right|_{[0,1]}$, we conclude that ind $Y=0$ and $i n d Z=0$; therefore, they are hereditarily disconnected ([30] Hausdorff, [1914]), and being linearly ordered, are strongly zero-dimensional ([31] Herrlich, [1965]), which is equivalent to Ind $Y=0$ and $\operatorname{Ind} Z=0$ ([23] Engelking, [1977]). Then for $\left.R_{\alpha}\right|_{[0,1]}=Y \cup Z$ we obtain $\left.\operatorname{Ind} R_{\alpha}\right|_{[0,1]} \leq 1$.

Clearly, since $\left.R_{\alpha}\right|_{[0,1]}$ is continuous then ind $\left.R_{\alpha}\right|_{[0,1]}=1$ and hence, by $1=\left.\operatorname{ind} R_{\alpha}\right|_{[0,1]} \leq\left.\operatorname{Ind} R_{\alpha}\right|_{[0,1]}=1$ and $\left.\operatorname{dim} R_{\alpha}\right|_{[0,1]} \leq\left.\operatorname{Ind} R_{\alpha}\right|_{[0,1]}$, we obtain a desired equality $\left.\operatorname{dim} R_{\alpha}\right|_{[0,1]}=1$. Otherwise, if $\left.\operatorname{dim} R_{\alpha}\right|_{[0,1]}=0$, then Ind $\left.R_{\alpha}\right|_{[0,1]}=0$, which is false, because $\left.\operatorname{Ind} R_{\alpha}\right|_{[0,1]}=1$.

The nature of the 1-dimensional manifold $\left.R_{\alpha}\right|_{[0,1]}$ (a generalized real number unit interval) which comes from the real number unit interval $\left.R_{\omega}\right|_{[0,1]}$ is illustrated by the following figures, where $\alpha=\omega \cdot 2$. The first figure is a bifurcation of a rational number, for a example, $x=\frac{1}{2}$ : 


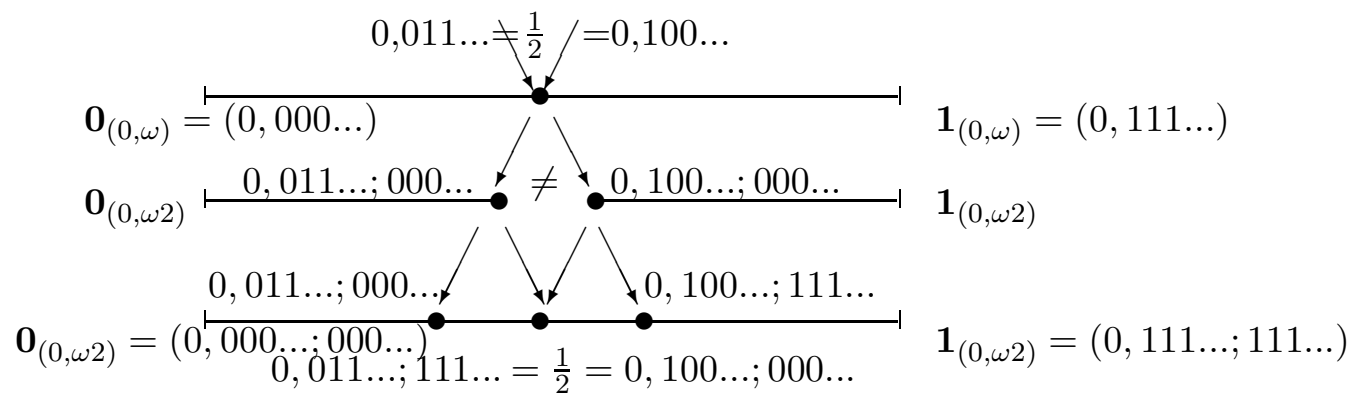

Fig. 2

and the second figure is a bifurcation of an irrational number, for example, $\frac{1}{\pi}$ :

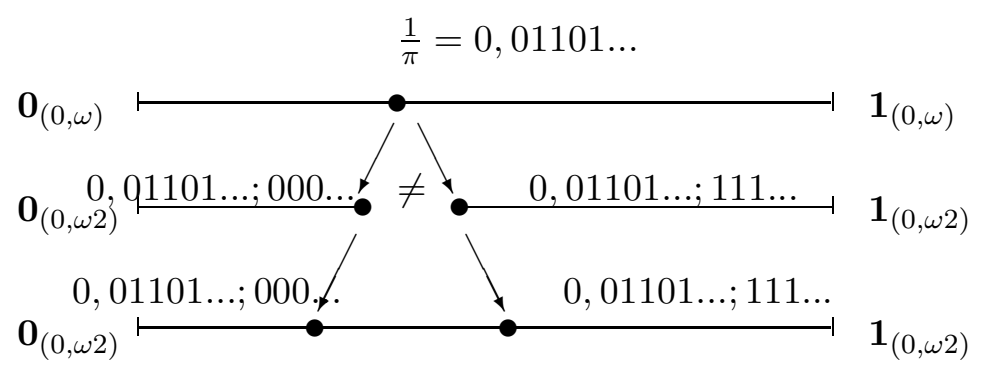

Fig. 3

We can also demonstrate a canonical embedding $i_{\omega}^{\omega \cdot 2}:\left.\left.R_{\omega}\right|_{[0,1]} \rightarrow R_{\omega \cdot 2}\right|_{[0,1]}$ which preserves the linear ordering, given by $i_{\omega}^{\omega \cdot 2}\left(X_{(0, \omega)}\right)=Y_{(0, \omega \cdot 2)}$, where $Y_{\beta}=X_{\beta}, 0 \leq \beta<\omega$, and $Y_{\beta}=0, \omega \leq \beta<\omega \cdot 2$.

Moreover, for each $\alpha=\omega \cdot \nu$ and $\beta=\omega \cdot \mu$, where $\nu \leq \mu$, there is a canonical embedding $i_{\alpha}^{\beta}:\left.\left.R_{\alpha}\right|_{[0,1]} \rightarrow R_{\beta}\right|_{[0,1]}$ which preserves the linear ordering, given by $i_{\alpha}^{\beta}\left(X_{0, \alpha}\right)=Y_{(0, \beta)}$, where $Y_{\gamma}=X_{\gamma}, 0 \leq \gamma<\alpha$, and $Y_{\gamma}=0, \alpha \leq \gamma<\beta$.

We can also formally consider the case where $\alpha=\boldsymbol{\Omega}=\omega \cdot \boldsymbol{\Omega}$ and the rational skands $X_{(0, \Omega)}$, which are eventually 0 or 1 , form a proper class $\left.Q_{\boldsymbol{\Omega}}\right|_{[0,1]}$ in $N B G^{-}$. As to $\left.R_{\boldsymbol{\Omega}}\right|_{[0,1]}$, i.e., the union of rational and irrational skands $X_{(0, \Omega)}$, it is not an object in $N B G^{-}$because irrational skands are 
elements of a super-class and outside of $N B G^{-}$-type theory. Nevertheless, one can consider a canonical embedding $i_{\alpha}^{\Omega}:\left.\left.R_{\alpha}\right|_{[0,1]} \rightarrow R_{\Omega}\right|_{[0,1]}$ which preserves the linear ordering, given by $i_{\alpha}^{\boldsymbol{\Omega}}\left(X_{(0, \alpha)}\right)=Y_{(0, \boldsymbol{\Omega})}$, where $Y_{\gamma}=X_{\gamma}$, $0 \leq \gamma<\alpha$, and $Y_{\gamma}=0, \alpha \leq \gamma<\boldsymbol{\Omega}$. Moreover, $\left.Q_{\boldsymbol{\Omega}}\right|_{[0,1]}=\bigcup_{\alpha<\boldsymbol{\Omega}} i_{\alpha}^{\boldsymbol{\Omega}}\left(R_{\alpha} \mid[0,1]\right)$, where $\alpha=\omega \cdot \nu, 1 \leq \nu<\Omega$.

One can also prove that $\left.Q_{\boldsymbol{\Omega}}\right|_{[0,1]}$ and $\left.R_{\boldsymbol{\Omega}}\right|_{[0,1]}$ are dense in themselves; $\left.Q_{\boldsymbol{\Omega}}\right|_{[0,1]}$ is dense in $\left.R_{\boldsymbol{\Omega}}\right|_{[0,1]}$ and $\left.R_{\boldsymbol{\Omega}}\right|_{[0,1]}$ is continuous. It is also clear that ind $\left.Q_{\boldsymbol{\Omega}}\right|_{[0,1]}=0$ and ind $\left.\boldsymbol{R}_{\boldsymbol{\Omega}}\right|_{[0,1]}=0$; therefore, they are hereditarily disconnected, and being linearly ordered, are strongly zero-dimensional, which is equivalent to $\operatorname{Ind} Y=0$ and $\operatorname{Ind} Z=0$. Then for $\left.R_{\Omega}\right|_{[0,1]}=Y \cup Z$ we obtain Ind $\left.R_{\Omega}\right|_{[0,1]} \leq 1$. Clearly, since $\left.R_{\Omega}\right|_{[0,1]}$ is continuous, then ind $\left.R_{\Omega}\right|_{[0,1]}=1$ and hence, by $1=$ ind $\left.R_{\Omega}\right|_{[0,1]} \leq\left.\operatorname{Ind} R_{\Omega}\right|_{[0,1]}=1$ and $\left.\operatorname{dim} R_{\boldsymbol{\Omega}}\right|_{[0,1]} \leq\left.\operatorname{Ind} R_{\boldsymbol{\Omega}}\right|_{[0,1]}$, we obtain a desired equality $\left.\operatorname{dim} R_{\boldsymbol{\Omega}}\right|_{[0,1]}=1$. Otherwise, if $\left.\operatorname{dim} R_{\Omega}\right|_{[0,1]}=0$, then $\left.\operatorname{Ind} R_{\Omega}\right|_{[0,1]}=0$, which is false, because Ind $\left.R_{\Omega}\right|_{[0,1]}=1$.

Unfortunately, all these natural arguments cannot be applied, because all references are to results valid only for topological spaces which are sets, and there are no similar results (even definitions of topology, dimensions, normality, etc.) for point proper classes, and it is not clear how to pass this gap. We are in a situation where something is evident, but there are no resources to prove it. We can only formulate the following conjecture.

Conjecture 3. $\left.R_{\boldsymbol{\Omega}}\right|_{[0,1]}$ is a maximally dense one-dimensional continuum whose elements (mostly hyper-classes) are limits of $\left.Q_{\boldsymbol{\Omega}}\right|_{[0,1]}$, i.e., limits of $\boldsymbol{\Omega}$-sequences whose terms are sets.

\section{Additive and multiplicative operations on generalized frac- tions}

In the next paragraph we shall formally extend for each ordinal $\alpha=\omega \nu$, $1 \leq \nu \leq \Omega$, the closed unit $\left.R_{\alpha}\right|_{[0,1]}$ to the set (hyper-class in the case $\nu=\boldsymbol{\Omega}) R_{\alpha}$ of generalized real numbers which is a one-dimensional linearly ordered continuous dense homogeneous point set (a dense homogeneous point manifold in the sense of Cantor). Unfortunately, only for $\nu=1$, i.e. $\alpha=\omega, R_{\omega}$ is supplied with addition and multiplication which are associative, commutative and distributive; moreover, $R_{\omega}=\mathbb{R}$ is the field of real numbers. If $\nu>1$, then it is not easy to completely define addition and multiplication in $R_{\alpha}$, although it has been tried in [6]. We introduce here our version, which is different both in notations and in intention from that in $[6]$.

For ordinal numbers there is a commutative and associative natural sum and a natural product in the sense of Hessenberg ([32], 591-594); i.e., if 
ordinal numbers $\xi$ and $\eta$ are represented in the form of normal expansion $\xi=\omega^{\xi_{1}} n_{1}+\omega^{\xi_{2}} n_{2}+\ldots+\omega^{\xi_{r}} n_{r}$ and $\eta=\omega^{\xi_{1}} m_{1}+\omega^{\xi_{2}} m_{2}+\ldots+\omega^{\xi_{r}} m_{r}$, respectively, where $\xi_{1}>\xi_{2}>\ldots>\xi_{r}$ are ordinal numbers, $n_{1}, n_{2}, \ldots, n_{r}$ and $m_{1}, m_{2}, \ldots, m_{r}$ are integers $\geq 0$, then by definition the natural sum is the following ordinal number:

$$
\xi \oplus \eta=\omega^{\xi_{1}}\left(n_{1}+m_{1}\right)+\omega^{\xi_{2}}\left(n_{2}+m_{2}\right)+\ldots+\omega^{\xi_{r}}\left(n_{r}+m_{r}\right) .
$$

In order to define the natural product $\alpha \odot \beta$ of the ordinal numbers $\alpha$ and $\beta$ one multiplies their normal expansions as if they were polynomials of variable $\omega$; multiplying two powers of number $\omega$, one forms the natural sum of the exponents and arranges the terms obtained from the multiplication according to decreasing exponents.

(Notice that instead of $\omega$ we can consider any ordinal number $\gamma>1$ as a basis of normal expansion of $\xi$ with $0 \leq n_{i}<\gamma, 1 \leq i \leq r$, see [36], Chap. XII, $\S 7$, e.g., $\gamma=2$; we use shall it below.)

Thus, the problem of the existence of the desired algebraic operations on $R_{\alpha}$ concerns only generalized real fractions of $\left.R_{\alpha}\right|_{[0,1]}$.

So addition "+", subtraction "-", multiplication "." and division "/" are not defined for all generalized real fractions $X_{(0, \alpha)}$ and $Y_{(0, \alpha)}$ in $\left.R_{\alpha}\right|_{[0,1]}$ but are defined for some of them. Here are typical cases of such a possibility:

$1)$. For every $X_{(0, \alpha)},\left.Y_{(0, \alpha)} \in R_{\alpha}\right|_{[0,1]}$, such that $X_{\alpha^{\prime}} \leq Y_{\alpha^{\prime}}, 0 \leq \alpha^{\prime}<\alpha$, we put $Y_{(0, \alpha)}-X_{(0, \alpha)}=Z_{(0, \alpha)}$, where $Z_{\alpha^{\prime}}=Y_{\alpha^{\prime}}-X_{\alpha^{\prime}}, 0 \leq \alpha^{\prime}<\alpha$. E.g., $X_{(0, \alpha)}-X_{(0, \alpha)}=0_{(0, \alpha)}$ or, for each $\left.X_{(0, \alpha)} \in R_{\alpha}\right|_{[0,1]}, 1_{[0,1]}-X_{(0, \alpha)}$ is always well defined.

If $Y_{(0, \alpha)}=\frac{1}{2^{\alpha^{\prime}}}$ and $X_{(0, \alpha)}=\frac{1}{2^{\alpha^{\prime \prime}}}, 0 \leq \alpha^{\prime}<\alpha^{\prime \prime}<\alpha$, the subtraction $\frac{1}{2^{\alpha^{\prime}}}-\frac{1}{2^{\alpha^{\prime \prime}}}$ is well defined. Indeed, in the case when $\alpha^{\prime}, \alpha^{\prime \prime}$ are ordinal numbers of the 2nd kind $\frac{1}{2^{\alpha^{\prime}}}-\frac{1}{2^{\alpha^{\prime \prime}}}=Z_{(0, \alpha)}$, where $Z_{\beta}=1$, for $\alpha^{\prime} \leq \beta<\alpha^{\prime \prime}$, and $Z_{\beta}=0$, for $\beta<\alpha^{\prime}$ or $\beta \geq \alpha^{\prime \prime}$; in the case when $\alpha^{\prime}$, $\alpha^{\prime \prime}$ are ordinal numbers of the 1st kind $\frac{1}{2^{\alpha^{\prime}}}-\frac{1}{2^{\alpha^{\prime \prime}}}=Z_{(0, \alpha)}$, where $Z_{\beta}=1$, for $\alpha^{\prime}<\beta \leq \alpha^{\prime \prime}$, and $Z_{\beta}=0$, for $\beta \leq \alpha^{\prime}$ or $\beta>\alpha^{\prime \prime}$; and at last in the case when $\alpha^{\prime}$ is an ordinal of the 1st kind and $\alpha^{\prime \prime}$ is an ordinal of the 2nd kind $\frac{1}{2^{\alpha^{\prime}}}-\frac{1}{2^{\alpha^{\prime \prime}}}=Z_{(0, \alpha)}$, where $Z_{\beta}=1$, for $\alpha^{\prime}<\beta<\alpha^{\prime \prime}$, and $Z_{\beta}=0$, for $\beta \leq \alpha^{\prime}$ or $\beta \geq \alpha^{\prime \prime}$. (Note that we use another notation in an appropriate case for twins.)

We will say that the interval $\left[X_{(0, \alpha)}, Y_{(0, \alpha)}\right]$ is of length $\frac{1}{2^{\alpha^{\prime}}}$, if $Y_{(0, \alpha)}-$ $X_{(0, \alpha)}=\frac{1}{2^{\alpha^{\prime}}}, 0 \leq \alpha^{\prime}<\alpha$.

$2)$. We can define $X_{(0, \alpha)}+Y_{(0, \alpha)}$ in the case where, for each $0 \leq \alpha^{\prime}<\alpha$, $X_{\alpha^{\prime}}$ and $Y_{\alpha^{\prime}}$ are both 0 , or one of them is 1 and another one is 0 . Then the result is $Z_{(0, \alpha)}=X_{(0, \alpha)}+Y_{(0, \alpha)}$ such that $Z_{\alpha^{\prime}}=X_{\alpha^{\prime}}+Y_{\alpha^{\prime}}, 0 \leq \alpha^{\prime}<\alpha$. In particular, if $Z_{(0, \alpha)}=Y_{(0, \alpha)}-X_{(0, \alpha)}$, then $X_{(0, \alpha)}+Z_{(0, \alpha)}=Z_{(0, \alpha)}+X_{(0, \alpha)}=$ $Y_{(0, \alpha)}$. 
If $\alpha^{\prime}$ is an ordinal of the 1st kind, then $\frac{1}{2^{\alpha^{\prime}}}+\frac{1}{2^{\alpha^{\prime}}}=\frac{1}{2^{\alpha^{\prime}-1}}, 0 \leq \alpha^{\prime}<\alpha$; and if $1 \leq \alpha^{\prime}<\alpha^{\prime \prime}<\alpha$, are arbitrary ordinal numbers of the 1st kind, then $\frac{1}{2^{\alpha^{\prime}}}+\frac{1}{2^{\alpha^{\prime \prime}}}=\frac{1}{2^{\alpha^{\prime \prime}}}+\frac{1}{2^{\alpha^{\prime}}}$ is $Z_{(0, \alpha)}$ such that $Z_{\beta}=1$, for $\beta=\alpha^{\prime}, \alpha^{\prime \prime}$; otherwise $Z_{\beta}=0$. If $0 \leq \alpha^{\prime \prime}<\alpha$ is an ordinal of the 2nd kind and $0 \leq \alpha^{\prime}<\alpha^{\prime \prime}<\alpha$ is an ordinal of the 1st kind, then $\frac{1}{2^{\alpha^{\prime}}}+\frac{1}{2^{\alpha^{\prime \prime}}}=\frac{1}{2^{\alpha^{\prime \prime}}}+\frac{1}{2^{\alpha^{\prime}}}=Z_{(0, \alpha)}$ such that $Z_{\beta}=1$, for $\beta=\alpha^{\prime}$ and $\beta \geq \alpha^{\prime \prime}$; otherwise, $Z_{\beta}=0$.

On the other hand, there are no magnitudes in $\left.R_{\alpha}\right|_{[0,1]}$ such as $\frac{1}{2^{\alpha^{\prime}}}+\frac{1}{2^{\alpha^{\prime}}}$, for every $0 \leq \alpha^{\prime}<\alpha$ which is an ordinal number of the 2 nd kind, because there is no ordinal number $\alpha^{\prime}-1$; as well as $\frac{1}{2^{\alpha^{\prime}}}+\frac{1}{2^{\alpha^{\prime \prime}}}$, if $\alpha^{\prime}$ is an ordinal number of the 2 nd kind and $\alpha^{\prime}<\alpha^{\prime \prime}$.

Moreover, we can define addition $X_{(0, \alpha)}+Y_{(0, \alpha)}$ in all cases which avoid the addition of components of the latter cases when sums of dyadic fractions do not exist.

3). For $1 \leq \alpha^{\prime}<\alpha$, where $\alpha^{\prime}$ is an ordinal number of the 1st kind, $\sum_{\alpha^{\prime} \leq \beta<\alpha^{\prime}+\omega} \frac{1}{2^{\beta}}=\frac{1}{2^{\alpha^{\prime}-1}}-\frac{1}{2^{\alpha^{\prime}+\omega}}$, where by an infinite sum we understand the supremum of finite sums, if of course they are well defined. Indeed, $\frac{1}{2^{\alpha^{\prime}}}+\frac{1}{2^{\alpha^{\prime}+1}}+\ldots+\frac{1}{2^{\alpha^{\prime}+n}}+\ldots=\sup _{n}\left(\frac{1}{2^{\alpha^{\prime}}}+\frac{1}{2^{\alpha^{\prime}+1}}+\ldots+\frac{1}{2^{\alpha^{\prime}+n}}\right)=\sup _{n}\left[\left(\frac{1}{2^{\alpha^{\prime}-1}}-\right.\right.$ $\left.\left.\frac{1}{2^{\alpha^{\prime}}}\right)+\left(\frac{1}{2^{\alpha^{\prime}+1}}-\frac{1}{2^{\alpha^{\prime}}}\right)+\ldots+\left(\frac{1}{2^{\alpha^{\prime}+n-1}}-\frac{1}{2^{\alpha^{\prime}+n}}\right)\right]=\sup _{n}\left(\frac{1}{2^{\alpha^{\prime}}-1}-\frac{1}{2^{\alpha^{\prime}+n}}\right)=\frac{1}{2^{\alpha^{\prime}-1}}-$ $\inf _{n} \frac{1}{2^{\alpha^{\prime}+n}}=\frac{1}{2^{\alpha^{\prime}-1}}-\frac{1}{2^{\alpha^{\prime}+\omega}}$.

4). We also put $\frac{1}{2^{\alpha^{\prime \prime}}} \cdot \frac{1}{2^{\alpha^{\prime}}}=\frac{1}{2^{\alpha^{\prime \prime} \oplus \alpha^{\prime}}}, 0 \leq \alpha^{\prime}, \alpha^{\prime \prime}<\alpha$.

$5)$. Each $\left.X_{(0, \alpha)} \in R_{\alpha}\right|_{[0,1]}$ can be divided by 2 . Indeed, it is an immediate consequence of the following propositions.

Theorem 7 Each $\left.X_{(0, \alpha)} \in R_{\alpha}\right|_{[0,1]}$, in particular, $0=0_{(0, \alpha)}$ and $1=$ $1_{(0, \alpha)}$ with 0 and 1 in all places, respectively, can be represented by the following formula:

$$
X_{(0, \alpha)}=\sum_{0 \leq \alpha^{\prime}<\alpha} \frac{F\left(\alpha^{\prime}\right)}{2^{\alpha^{\prime}+1}}
$$

where the summation is taken over all ordinals $0 \leq \alpha^{\prime}<\alpha$, and $F\left(\alpha^{\prime}\right)$ is equal to the element of the component $X_{\alpha^{\prime}}$ of $X_{(0, \alpha)}$. In particular, for $0 \leq \alpha_{0}<\alpha$, we obtain the following formula:

$$
\frac{1}{2^{\alpha_{0}}}=\sum_{\alpha_{0} \leq \alpha^{\prime}<\alpha} \frac{1}{2^{\alpha^{\prime}+1}}=\frac{1}{2^{\alpha_{0}+1}}+\frac{1}{2^{\alpha_{0}+2}}+\ldots+\frac{1}{2^{\omega+1}}+\ldots+\frac{1}{2^{\alpha^{\prime}+1}}+\ldots
$$

The Proof is an immediate consequence of the above definitions.

Thus, we can divide each $\left.X_{(0, \alpha)} \in R_{\alpha}\right|_{[0,1]}$ by 2 , changing each term $\frac{1}{2^{\beta}}$ in (82) by $\frac{1}{2^{\beta+1}}$ and adding summands $\frac{1}{2^{\omega \eta+1}}$, if $X_{\beta}=1, \alpha^{\prime} \leq \beta<\alpha^{\prime}+\omega \nu$, for some $0 \leq \alpha^{\prime}<\alpha$, where $\alpha^{\prime}$, is an ordinal number of the 2 nd kind such 
that $\alpha^{\prime}+\omega \nu<\alpha, 1 \leq \eta<\nu$, and the sum of all changed terms is the result $Z_{(0, \alpha)}=X_{(0, \alpha)} / 2=X_{(0, \alpha)} \cdot \frac{1}{2}$.

Lemma 6. Each interval $\left[X_{(0, \alpha)}, Y_{(0, \alpha)}\right]$ of length $\frac{1}{2^{\alpha^{\prime}}}, 0 \leq \alpha^{\prime}<\alpha$, can be halved.

Proof. In fact, $Y_{(0, \alpha)}-X_{(0, \alpha)}=\frac{1}{2^{\alpha^{\prime}}}$ or $Y_{(0, \alpha)}=X_{(0, \alpha)}+\frac{1}{2^{\alpha^{\prime}}}=X_{(0, \alpha)}+$ $\frac{1}{2^{\alpha^{\prime}+1}}+\frac{1}{2^{\alpha^{\prime}+1}}$. Consequently, $Y_{(0, \alpha)}-\frac{1}{2^{\alpha^{\prime}+1}}=X_{(0, \alpha)}+\frac{1}{2^{\alpha^{\prime}+1}}$ and hence $\left[X_{(0, \alpha)}, X_{(0, \alpha)}+\frac{1}{2^{\alpha^{\prime}}}\right] \cup\left[Y_{(0, \alpha)}-\frac{1}{2^{\alpha^{\prime}}}, Y_{(0, \alpha)}\right]=\left[X_{(0, \alpha)}, Y_{(0, \alpha)}\right]$. Moreover, lengths of $\left[X_{(0, \alpha)}, X_{(0, \alpha)}+\frac{1}{2^{\alpha^{\prime}}+1}\right]$ and $\left[Y_{(0, \alpha)}-\frac{1}{2^{\alpha^{\prime}+1}}, Y_{(0, \alpha)}\right]$ are equal to $\frac{1}{2^{\alpha^{\prime}+1}}=\frac{1}{2^{\alpha^{\prime}}} \cdot \frac{1}{2}$.

Although there are no magnitudes in $\left.R_{\alpha}\right|_{[0,1]}$ like $\frac{n}{2^{\alpha^{\prime}}}$, for each $2 \leq n<$ $\omega$, where $0 \leq \alpha^{\prime}<\alpha$ is an ordinal number of the 2 nd kind, there are magnitudes in $\left.R_{\alpha}\right|_{[0,1]}$ of multiplications $\frac{1}{2^{\beta}} \cdot 2^{\alpha^{\prime}}$ for some ordinals $1 \leq$ $\beta, \alpha^{\prime}<\alpha=\omega \nu, \nu \geq 1$, e.g., $\frac{1}{2^{\omega}} \cdot 2^{\omega}=1_{(0, \alpha)}$, which is really unexpected.

Proposition 24. For each $\alpha^{\prime}, \alpha^{\prime \prime} \in$ On, $0 \leq \alpha^{\prime}, \alpha^{\prime \prime}<\alpha=\omega \nu, \nu \geq 1$, the following formula

$$
\frac{1}{2^{\alpha^{\prime}}}=\frac{1}{2^{\alpha^{\prime} \oplus \omega^{\prime \prime}}} \cdot 2^{\alpha^{\prime \prime}}
$$

holds; in particular, $\frac{1}{\omega} \cdot \omega=\frac{1}{2^{\omega}} \cdot 2^{\omega}=1_{(0, \alpha)}$.

Proof. Since $\frac{1}{2^{\alpha^{\prime}}} \cdot \frac{1}{2^{\alpha^{\prime \prime}}} \stackrel{\text { def }}{=} \frac{1}{2^{\alpha^{\prime} \oplus \omega^{\prime \prime}}}$ it is sufficient to show that $\frac{1}{2^{\alpha^{\prime \prime}}} \cdot 2^{\alpha^{\prime \prime}}=1$. By Proposition 24, we obtain the following identities:

$$
\begin{aligned}
& 1=\frac{1}{2^{0}}=\frac{1}{2}+\frac{1}{2^{2}}+\frac{1}{2^{3}}+\ldots=\left(\frac{1}{2^{2}}+\frac{1}{2^{3}}+\ldots\right)+\left(\frac{1}{2^{2}}+\frac{1}{2^{3}}+\ldots\right)= \\
& \left(\frac{1}{2^{2}}+\frac{1}{2^{3}}+\ldots\right) \cdot 2=\frac{1}{2} \cdot 2=\left[\left(\frac{1}{2^{3}}+\frac{1}{2^{4}}+\ldots\right)+\left(\frac{1}{2^{3}}+\frac{1}{2^{4}}+\ldots\right)\right] \cdot 2= \\
& \left(\frac{1}{2^{3}}+\frac{1}{2^{4}}+\ldots\right) \cdot 2^{2}=\frac{1}{2^{2}} \cdot 2^{2}=\ldots=\left[\left(\frac{1}{2^{n+1}}+\frac{1}{2^{n+2}}+\ldots\right)+\right. \\
& \left.\left(\frac{1}{2^{n+1}}+\frac{1}{2^{n+2}}+\ldots\right)\right] \cdot 2^{n-1}=\left(\frac{1}{2^{n+1}}+\frac{1}{2^{n+2}}+\ldots\right) \cdot 2^{n-1}+ \\
& \left(\frac{1}{2^{n+1}}+\frac{1}{2^{n+2}}+\ldots\right) \cdot 2^{n-1}=\left(\frac{1}{2^{n+1}}+\frac{1}{2^{n+2}}+\ldots\right) \cdot 2^{n}=\frac{1}{2^{n}} \cdot 2^{n}= \\
& \left(\frac{1}{2^{n}}-\frac{1}{2^{\omega}}+\frac{1}{2^{\omega+1}}+\frac{1}{2^{\omega+2}}+\ldots\right) \cdot 2^{n}=\ldots= \\
& =\left(\frac{1}{2^{\omega}}-\frac{1}{2^{\omega}}+\frac{1}{2^{\omega+1}}+\frac{1}{2^{\omega+2}}+\ldots\right) \cdot 2^{\omega}= \\
& \left(\frac{1}{2^{\omega+1}}+\frac{1}{2^{\omega+2}}+\ldots\right) \cdot 2^{\omega}=\frac{1}{2^{\omega}} \cdot 2^{\omega}=\ldots= \\
& =\left(\frac{1}{2^{\alpha^{\prime \prime}+1}}+\frac{1}{2^{\alpha^{\prime \prime}+2}}+\ldots\right) \cdot 2^{\alpha^{\prime \prime}}=\frac{1}{2^{\alpha^{\prime \prime}}} \cdot 2^{\alpha^{\prime \prime}} .
\end{aligned}
$$

We also need the following lemma.

Lemma 7. For every $X_{(0, \alpha)}<Y_{(0, \alpha)}$ in $\left.R_{\alpha}\right|_{[0,1]}$ there are $X_{(0, \alpha)}^{\prime}<Y_{(0, \alpha)}^{\prime}$ in $\left.Q_{\alpha}\right|_{[0,1]}$ such that $X_{(0, \alpha)}<X_{(0, \alpha)}^{\prime}, Y_{(0, \alpha)}^{\prime}<Y_{(0, \alpha)}$ and $Y_{(0, \alpha)}^{\prime}-X_{(0, \alpha)}^{\prime}=\frac{1}{2^{\alpha^{\prime}}}$ for some $2 \leq \alpha^{\prime}<\alpha$; in particular, for each $Y_{(0, \alpha)}$ there is $\frac{1}{2^{\alpha^{\prime}}}$ such that $\frac{1}{2^{\alpha^{\prime}}}<Y_{(0, \alpha)}$.

Proof. Since $X_{(0, \alpha)}<Y_{(0, \alpha)}$ there exists $\alpha^{\prime}, 0 \leq \alpha^{\prime}<\alpha$ such that $X_{\alpha^{\prime}}=0, Y_{\alpha^{\prime}}=1$ and $X_{\beta}=Y_{\beta}$, for all $0 \leq \beta<\alpha^{\prime}$. There is a minimal $\alpha^{\prime \prime}>\alpha^{\prime}$ such that $X_{\alpha^{\prime \prime}}=0$; otherwise, $X_{\beta}=1$, for all $\beta>\alpha^{\prime}$ and hence 
$X_{\alpha^{\prime}}$ should be equal to 1 . Consider $X_{(0, \alpha)}^{\prime}$ such that $X_{\alpha^{\prime \prime}}^{\prime}=1, X_{\beta}^{\prime}=X_{\beta}$, $0 \leq \beta<\alpha^{\prime \prime}$, and $X_{\beta}^{\prime}=0, \beta>\alpha^{\prime \prime}$. Clearly, $X_{(0, \alpha)}<X_{(0, \alpha)}^{\prime}<Y_{(0, \alpha)}$. Consider $Y_{(0, \alpha)}^{\prime}$ such that $Y_{\alpha^{\prime \prime}+1}^{\prime}=1$ and $Y_{\beta}^{\prime}=X_{\beta}^{\prime}, 0 \leq \beta \leq \alpha^{\prime \prime}$. Clearly, $X_{(0, \alpha)}^{\prime}<Y_{(0, \alpha)}^{\prime}<Y_{(0, \alpha)}$ and $Y_{(0, \alpha)}^{\prime}-X_{(0, \alpha)}^{\prime}=\frac{1}{2^{\alpha^{\prime \prime}+1}}$, i.e., $\alpha^{\prime}=\alpha^{\prime \prime}+1$.

Definition 20. Let $X_{(0, \alpha)}$ and $Y_{(0, \alpha)}$ be two elements of $\left.R_{\alpha}\right|_{[0,1]}$ such that $X_{(0, \alpha)} \leq Y_{(0, \alpha)}$ and $Y_{(0, \alpha)}-X_{(0, \alpha)}$ is defined. Then the generalized real number $l_{(0, \alpha)}=Y_{(0, \alpha)}-X_{(0, \alpha)}$ is called a length of the closed interval $\left[X_{(0, \alpha)}, Y_{(0, \alpha)}\right]$ and of the open interval $\left(X_{(0, \alpha)}, Y_{(0, \alpha)}\right)$.

Theorem 8. Let $\left[X_{(0, \alpha)}, Y_{(0, \alpha)}\right] \supset\left[X_{(0, \alpha)}^{1}, Y_{(0, \alpha)}^{1}\right] \supset \ldots \supset\left[X_{(0, \alpha)}^{\alpha^{\prime}}, Y_{(0, \alpha)}^{\alpha^{\prime}}\right] \supset$ ... be a system of embedded closed intervals of $\left.R_{\alpha}\right|_{[0,1]}$ such that $\inf _{\alpha^{\prime}} l_{(0, \alpha)}^{\alpha^{\prime}}=$ $0_{(0, \alpha)}$; then there exists a unique element $\left.Z_{(0, \alpha)} \in R_{\alpha}\right|_{[0,1]}$ such that it belongs to all these intervals, i.e., $\bigcap_{\alpha^{\prime}}\left[X_{(0, \alpha)}^{\alpha^{\prime}}, Y_{(0, \alpha)}^{\alpha^{\prime}}\right]=Z_{(0, \alpha)}$.

Proof. By Theorem 5, there exist $\bar{M}_{(0, \alpha)}=\sup _{0 \leq \alpha^{\prime}<\alpha}\left\{X_{(0, \alpha)}^{\alpha^{\prime}}\right\}$ and $\bar{m}_{(0, \alpha)}=$ $\inf _{0 \leq \alpha^{\prime}<\alpha}\left\{Y_{(0, \alpha)}^{\alpha^{\prime}}\right\}$ in $\left.R_{\alpha}\right|_{[0,1]}$. Then $Z_{(0, \alpha)}=\bar{M}_{(0, \alpha)}=\bar{m}_{(0, \alpha)}$. Otherwise, by Lemma 7, for $\bar{m}_{(0, \alpha)}<\bar{M}_{(0, \alpha)}$ in $\left.R_{\alpha}\right|_{[0,1]}$ there are $X_{(0, \alpha)}^{\prime}<Y_{(0, \alpha)}^{\prime}$ in $\left.Q_{\alpha}\right|_{[0,1]}$ such that $\bar{m}_{(0, \alpha)}<X_{(0, \alpha)}^{\prime}<Y_{(0, \alpha)}^{\prime}<\bar{M}_{(0, \alpha)}$ and $Y_{(0, \alpha)}^{\prime}-X_{(0, \alpha)}^{\prime}=\frac{1}{2^{\alpha^{\prime}}}$ for some $2 \leq \alpha^{\prime}<\alpha$, which is in contradiction with the assumption that $\inf _{\alpha^{\prime}} l_{(0, \alpha)}^{\alpha^{\prime}}=0_{(0, \alpha)}$, because $\left[X_{(0, \alpha)}^{\prime}, Y_{(0, \alpha)}^{\prime}\right] \subset\left[X_{(0, \alpha)}^{\alpha^{\prime}}, Y_{(0, \alpha)}^{\alpha^{\prime}}\right]$, for each $0 \leq \alpha^{\prime}<$ $\alpha$.

Theorem 9. $\left.R_{\alpha}\right|_{[0,1]}$ in the order topology is a compact Hausdorff space.

Proof. We already know that the linearly ordered space $\left.R_{\alpha}\right|_{[0,1]}$ is normal. Let now $\gamma=\left\{U_{\lambda}\right\} \mid \lambda \in \Lambda$ be an arbitrary covering of $\left.R_{\alpha}\right|_{[0,1]}$, consisting of open intervals $U_{\lambda}$ of $\left.R_{\alpha}\right|_{[0,1]}$. We have to prove that there exists a finite subcovering of $\gamma$, which covers $\left.R_{\alpha}\right|_{[0,1]}$. Suppose the contrary, and we cannot choose such a finite subcovering. By Lemma 6 , we can halve $\left.R_{\alpha}\right|_{[0,1]}=\left[X_{(0, \alpha)}, Y_{(0, \alpha)}\right]$ (evidently, $X_{(0, \alpha)}=0_{(0, \alpha)}$ and $\left.Y_{(0, \alpha)}=1_{(0, \alpha)}\right)$ and choose one $\left[X_{(0, \alpha)}^{1}, Y_{(0, \alpha)}^{1}\right]$ of the parts that cannot be covered by finite elements of $\gamma$. Then we halve $\left[X_{(0, \alpha)}^{1}, Y_{(0, \alpha)}^{1}\right]$ and choose one $\left[X_{(0, \alpha)}^{2}, Y_{(0, \alpha)}^{2}\right]$ of the parts that cannot be covered by finite elements of $\gamma$. We continue this process and conclude that $\left[X_{(0, \alpha)}^{\omega}, Y_{(0, \alpha)}^{\omega}\right]$ cannot be also covered by finite elements of $\gamma$, otherwise, $\left[X_{(0, \alpha)}^{n}, Y_{(0, \alpha)}^{n}\right]$ can be covered by finite elements of $\gamma$, which is in contradiction with our choice. We halve $\left[X_{(0, \alpha)}^{\omega}, Y_{(0, \alpha)}^{\omega}\right]$ and continue our choice for each $0 \leq \alpha^{\prime}<\alpha$. We have gotten a system $\left[X_{(0, \alpha)}, Y_{(0, \alpha)}\right] \supset\left[X_{(0, \alpha)}^{1}, Y_{(0, \alpha)}^{1}\right] \supset \ldots \supset\left[X_{(0, \alpha)}^{\alpha^{\prime}}, Y_{(0, \alpha)}^{\alpha^{\prime}}\right] \supset \ldots$ of embedded closed intervals of $\left.R_{\alpha}\right|_{[0,1]}$ such that $\inf _{\alpha^{\prime}} l_{(0, \alpha)}^{\alpha^{\prime}}=0_{(0, \alpha)}$. Then, by Theorem 
8, there exists a unique element $\left.Z_{(0, \alpha)} \in R_{\alpha}\right|_{[0,1]}$ such that it belongs to all these intervals, i.e., $\bigcap_{\alpha^{\prime}}\left[X_{(0, \alpha)}^{\alpha^{\prime}}, Y_{(0, \alpha)}^{\alpha^{\prime}}\right]=Z_{(0, \alpha)}$. Since $\gamma$ is a covering of $\left.R_{\alpha}\right|_{[0,1]}$ there exists an element $U_{\lambda} \in \gamma$ such that $Z_{(0, \alpha)} \in U_{\lambda}$. Since $\inf _{\alpha^{\prime}} l_{(0, \alpha)}^{\alpha^{\prime}}=0_{(0, \alpha)}$ we conclude that there exists an ordinal number $\alpha^{\prime}<\alpha$ such that $\left[X_{(0, \alpha)}^{\alpha^{\prime}}, Y_{(0, \alpha)}^{\alpha^{\prime}}\right] \subset U_{\lambda}$ and one-element subcovering $U_{\lambda}$ of $\gamma$ covers $\left[X_{(0, \alpha)}^{\alpha^{\prime}}, Y_{(0, \alpha)}^{\alpha^{\prime}}\right]$, which is in contradiction with our choice. Thus the assumption that there is no finite subcovering of $\gamma$ which covers $\left.R_{\alpha}\right|_{[0,1]}$ is wrong.

\section{Generalized real numbers and generalized straight lines}

Now we are going to extend $\left.R_{\alpha}\right|_{[0,1]}, \alpha=\omega \nu, \nu \geq 1$, to the set $R_{\alpha}$ of all generalized real numbers.

By $R_{\alpha}^{+}$we denote $\left.R_{\alpha}\right|_{[0,1]} \cup\left(\left.R_{\alpha}\right|_{(0,1]}\right)^{*}$, where $(L)^{*}$ is the backwards linear ordering of $\left.L=\left.R_{\alpha}\right|_{(0,1]}=\left.R_{\alpha}\right|_{[0,1] \backslash\left\{0_{(1, \alpha)}\right.}\right\}$, and identify $1_{(0, \alpha)}$ and $\left(1_{(0, \alpha)}\right)^{*}$ with the ordinal 1 , and each ordinal $2^{\alpha^{\prime}}, 0<\alpha^{\prime}<\alpha$ with $\left(\frac{1}{2^{\alpha^{\prime}}}\right)^{*} \in L^{*}$ with the obvious ordering, adding to the following already defined relations: $Y<Z$ for each $Y \neq 1_{(1, \alpha)}$ in $L$ and each $Z \neq\left(1_{(0, \alpha)}\right)^{*}$ in $(L)^{*}$. Putting $R_{\alpha}^{-}=\left(R_{\alpha}^{+}\right)^{*}$ and denoting $\left(X_{(0, \alpha)}\right)^{*}$ by $-X_{(0, \alpha)}$, for every $X_{(0, \alpha)} \in R_{\alpha}^{+}$, identifying $0_{(1, \alpha)}$ and $-0_{(1, \alpha)}$, we define $R_{\alpha}=R_{\alpha}^{-} \cup R_{\alpha}^{+}$as generalized real numbers with the obvious ordering, adding to the following already defined relations: $X_{(0, \alpha)}<Y_{(0, \alpha)}$, for each $X_{(0, \alpha)} \neq-0_{(1, \alpha)}$ in $R_{\alpha}^{-}$and each $Y_{(0, \alpha)} \neq 0_{(1, \alpha)}$ in $R_{\alpha}^{+}$. It is clear that $R_{\alpha}$ is a set of the power $2^{|\alpha|}$.

Similarly, we can extend $\left.Q_{\alpha}\right|_{[0,1]}$ to the dense subordering subset $Q_{\alpha}$ of $R_{\alpha}$ and call it generalized rational numbers. Its cardinality is $\sum_{\alpha^{\prime}<\alpha} 2^{\left|\alpha^{\prime}\right|}$.

One can easily prove that $Q_{\alpha}$ is dense in $R_{\alpha} ; \operatorname{dim} Q_{\alpha}=0, \operatorname{dim}\left(R_{\alpha}\right)$ $\left.Q_{\alpha}\right)=0, \operatorname{dim} R_{\alpha}=1 ; R_{\alpha}$ is continuous, i.e., for every bounded set $X_{\alpha} \subset R_{\alpha}$, there exists an interval $\left[\alpha_{0}, \alpha_{1}\right]$ such that $X_{\alpha} \subseteq\left[\alpha_{0}, \alpha_{1}\right]$ has a smallest upper bound and a greatest lower bound; every closed bounded set is compact; each Dedekind section in $R_{\alpha}$ has no gap.

In the case $\alpha=\omega^{\kappa}, \kappa \geq 1$ we can represent $R_{\alpha}$ in a more natural form.

Definition 21. By a set $R_{\alpha}^{+}$of all non-negative generalized numbers we understand an extended system of embedded curly braces

$$
\cdots\left\{-\alpha^{\prime} \cdots\left\{-1\left\{0\left\{1 \cdots\left\{\alpha^{\prime} \cdots\right\}\right\}\right\}\right\}\right\}
$$

filled by 0 or 1 such that for each $X_{(-\alpha, \alpha)} \in R_{\alpha}^{+}, X_{\alpha^{\prime}}=1$ only for finite number indexes, $-\alpha<\alpha^{\prime}<0$. We consider on $R_{\alpha}^{+}$the lexicographic ordering identifying twins as above. If all non-negative places are filled by 0 we have the usual ordinal numbers in Cantor's normal form with base 2 
and the lexicographic ordering of them which coincides with the usual one. By a set $R_{\alpha}^{-}$of all non-positive generalized numbers we understand the backwards linear ordering $\left(R_{\alpha}^{+}\right)^{*}$, and denote $X_{(-\alpha, \alpha)}^{*} \in\left(R_{\alpha}^{+}\right)^{*}$ by $-X_{(-\alpha, \alpha)}$. At last, by a set $R_{\alpha}$ of all generalized numbers we understand $R_{\alpha}^{-} \cup R_{\alpha}^{+}$with the natural identification $0_{(-\alpha, \alpha)}^{+}$and $0_{(-\alpha, \alpha)}^{-}$and a clear linear ordering. Further we denote by $X_{(-\alpha, \alpha)}$ an arbitrary element of $R_{\alpha}$ which can be positive, negative or zero.

Theorem 10. If $\alpha=\omega^{\kappa}, \kappa \geq 1$, then $R_{\alpha}$ is topologically and order isomorphic to $R_{\alpha}$ in the sense of Definition 22.

Proof. We give only a sketch of a proof. It is enough to prove that $\left.R_{\alpha}^{+} \backslash R_{\alpha}\right|_{[0,1)}$ is isomorphic to $\left.R_{\alpha}^{+} \backslash R_{\alpha}\right|_{[0,1)}$ in the sense of Definition 22, because $\left.R_{\alpha}\right|_{[0,1)}$ are isomorphic in both senses: notice that $\left.R_{\alpha}\right|_{(0,1]}$ are also isomorphic in both senses.

Since $\left.R_{\alpha}\right|_{(0,1]}$ and $\left(\left.R_{\alpha}\right|_{(0,1]}\right)^{*}$ are evidently topologically isomorphic it is enough to show that $\left(\left.R_{\alpha}\right|_{(0,1]}\right)^{*}$ and $\left\{X_{(-\alpha, \alpha)} \in R_{\alpha} \mid X_{(-\alpha, \alpha)} \geq 1_{(-\alpha, \alpha)}\right\}$ or $\left.R_{\alpha}\right|_{(0,1]}$ and $\left\{X_{(-\alpha, \alpha)} \in R_{\alpha} \mid X_{(-\alpha, \alpha)} \geq 1_{(-\alpha, \alpha)}\right\}$ are topologically isomorphic, respectively.

This isomorphism can be defined by the following transfinite induction: the first step is to show that $\left.R_{\alpha}\right|_{\left[\frac{1}{2}, 1\right]}$ is isomorphic to $\left.R_{\alpha}\right|_{[1,2]}$. It can be done by putting in correspondence $\frac{1}{2}$ to 2 and 1 to 1 , respectively (we obviously simplify the notation). By halving the intervals $\left[\frac{1}{2}, 1\right]$ and $[1,2]$ we put their centers in correspondence to each other, i.e., $\frac{1}{2}+\frac{1}{2^{2}}$ to $1+\frac{1}{2}$, and do the same (i.e., halving the intervals) with each corresponding interval $\left[\frac{1}{2}, \frac{1}{2}+\frac{1}{2^{2}}\right]$ and $\left[1+\frac{1}{2}, 2\right]$ as well as $\left[\frac{1}{2}+\frac{1}{2^{2}}, 1\right]$ and $\left[1,1+\frac{1}{2}\right]$, respectively. Of course, the limit ends will be in this natural correspondence and we continue halving further and further, i.e., $\alpha$ times. It is clear that the closures of the corresponding isomorphic sets of halving are also isomorphic and they coincide with $\left.R_{\alpha}\right|_{\left[\frac{1}{2}, 1\right]}$ and $\left.R_{\alpha}\right|_{[1,2]}$, respectively.

We can do the same with $\left.R_{\alpha}\right|_{\left[\frac{1}{2^{2}}, 1\right]}$ and $\left.R_{\alpha}\right|_{\left[1,2^{2}\right]}$, respectively, and notice that a new isomorphism restricted on $\left.R_{\alpha}\right|_{\left[\frac{1}{2}, 1\right]}$ and $\left.R_{\alpha}\right|_{[1,2]}$, respectively, will coincide with the previous one. The limit isomorphism between $\left.R_{\alpha}\right|_{\left(\frac{1}{2 \omega}, 1\right]}$ and $\left.R_{\alpha}\right|_{\left[1,2^{\omega}\right)}$ is obvious, which we extend to the isomorphism between $\left.R_{\alpha}\right|_{\left[\frac{1}{2^{\omega}}, 1\right]}$ and $\left.R_{\alpha}\right|_{\left[1,2^{\omega}\right]}$.

In the same manner we show that $\left.R_{\alpha}\right|_{\left[\frac{1}{2^{\omega+n}}, \frac{1}{\left.2^{\omega}\right]}\right.}$ and $\left.R_{\alpha}\right|_{\left[2^{\omega}, 2^{\omega+n}\right]}, 1 \leq n<$ $\omega$, are isomorphic. (Only notice here that if $\alpha \neq \omega^{\kappa}, \kappa \geq 1$, then this step would be wrong.) And extend it to the isomorphism between $\left.R_{\alpha}\right|_{\left[\frac{1}{2^{2}}, \frac{1}{2^{\omega}}\right]}$ and $\left.R_{\alpha}\right|_{\left[2^{\omega}, 2^{\omega 2}\right]}$ and hence between $\left.R_{\alpha}\right|_{\left[\frac{1}{2^{\omega 2}}, 1\right]}$ and $\left.R_{\alpha}\right|_{\left[1,2^{\omega 2}\right]}$. The further 
steps are similar. We omit the details. The resultant ordering isomorphism between $R_{\alpha}$ in both senses will be an isomorphism, too.

If each element of $R_{(-\alpha, \alpha)}, \alpha=\omega_{\xi}, \xi \geq 0$, is considered as a geometric point, then we denote this point set by $L_{(-\alpha, \alpha)}$ and call it a generalized straight line. If $\xi=0$, then we obtain a classical Euclidean line, which as we know is uniquely defined by Hilbert's axioms. Moreover, by one of Euclid's definitions: "The straight line is a line such that it is uniformly arranged towards all its points". It is not so in the cases where $\xi>0$; i.e. there are different kinds of points, e.g., $\xi=1$ and $\frac{1}{2^{\omega}}$ and $\frac{1}{2^{\omega+1}}$ in $R_{\left(-\omega_{1}, \omega_{1}\right)}$, which have a different structure to the right of them, though the same structure to the left. We are going to state the following conjecture.

Conjecture 4. There exists a system of axioms including a generalized Archimedian axiom, a generalized Cantor's axiom of continuity, which uniquely defines the generalized straight line $L_{\left(-\omega_{\xi}, \omega_{\xi}\right)}, \xi \geq 1$, with an isomorphism $\varphi: L_{\left(-\omega_{\xi}, \omega_{\xi}\right)} \rightarrow R_{\left(-\omega_{\xi}, \omega_{\xi}\right)}$.

In favour of this conjecture says the following corollary of Proposition 26:

Corollary 10. For each generalized dyadic fraction $a=\frac{1}{2^{\alpha^{\prime}}}, \alpha^{\prime} \in \mathbf{O n}$, $1 \leq \alpha^{\prime}<\alpha=\omega_{\xi}, \xi \geq 1$, and arbitrary positive generalized real number $b \in R_{\left(-\omega_{\xi}, \omega_{\xi}\right)}^{+}$there exists an ordinal number $\nu, 1 \leq \nu<\omega_{\xi}$, such that $a \cdot \nu>b$.

Proof. Indeed, there exists an ordinal number $\beta, 0 \leq \beta<\alpha=\omega_{\xi}$, such that $2^{\beta}>[b]$, where $[b]$ is an integral part of $b$. Putting $\alpha^{\prime \prime}=\alpha^{\prime} \oplus \beta$, we obtain $\nu=2^{\alpha^{\prime} \oplus \beta}$ which, by Proposition 24, satisfies the desired condition, i.e., $a \cdot \nu=\frac{1}{2^{\alpha^{\prime}}} \cdot \nu=\frac{1}{2^{\alpha^{\prime}}} \cdot 2^{\alpha^{\prime} \oplus \beta}=\frac{1}{2^{\alpha^{\prime}}} \cdot 2^{\alpha^{\prime}} \odot 2^{\beta}=\left(\frac{1}{2^{\alpha^{\prime}}} \cdot 2^{\alpha^{\prime}}\right) \cdot 2^{\beta}=1 \cdot 2^{\beta}>b$.

Note also that the geometry of such straight lines is different from the classical one; i.e., $L_{(-\omega, \omega)}$. E.g., in the generalized plane, i.e., $L_{\left(-\omega_{\xi}, \omega_{\xi}\right)}^{2}=$ $L_{\left(-\omega_{\xi}, \omega_{\xi}\right)} \times L_{\left(-\omega_{\xi}, \omega_{\xi}\right)}, \xi \geq 1$, it is not a case that each of two different points of $R_{\left(-\omega_{\xi}, \omega_{\xi}\right)}^{2}$ belongs to a generalized line in $R_{\left(-\omega_{\xi}, \omega_{\xi}\right)}^{2}$. For example, there are straight lines in $R_{\left(-\omega_{\xi}, \omega_{\xi}\right)}^{2}$ such as $y=x$, or $y=-x$, or $x=$ const, or $y=$ const, but there is no line given by the following equation: $y=2 x$. So for possible straight lines in $R_{\left(-\omega_{\xi}, \omega_{\xi}\right)}^{2}, \xi \geq 1$, a generalized version of Zeno's paradox arises; however in the direction $y=2 x$, Zeno's arrow does not even exist; it is totally destroyed.

\section{Elements of a generalized calculus}

Definition 22. By an $\alpha^{\prime}$-sequence in $R_{\alpha}, \omega \leq \alpha^{\prime} \leq \alpha \leq \boldsymbol{\Omega}, \alpha=$ $\omega \nu, \alpha^{\prime}=\omega \nu^{\prime}, 1 \leq \nu, \nu^{\prime} \leq \boldsymbol{\Omega}$, we understand a generalized skand $S_{\left(0, \alpha^{\prime}\right)}$ whose components $S_{\beta^{\prime}}$ are elements of $R_{\alpha}, 0 \leq \beta^{\prime}<\alpha^{\prime}$. We denote it 
temporarily by $S_{\beta^{\prime}}, 0 \leq \beta^{\prime}<\alpha^{\prime}$; in the usual way its denotation is more complicated, i.e., $\left.\left\{S_{\beta^{\prime}}\right\}\right|_{\beta^{\prime}<\alpha^{\prime}}$ and is called a transfinite sequence of type $\alpha^{\prime}$ (see the corresponding definitions of course for transfinite sequences of ordinal numbers [52], p. 287).

Remark 21. So, for $\alpha>\omega$, there are many converging $\alpha^{\prime}$-sequences in $R_{\alpha}, \omega \leq \alpha^{\prime} \leq \alpha$, where $\alpha^{\prime}=\omega \nu, \nu \geq 1$. Indeed, since each generalized real number $X_{(-\alpha, \alpha)} \in R_{\alpha}$ is the intersection of some $\alpha^{\prime}$-sequence of embedded closed intervals $\left[X_{(-\alpha, \alpha)}, B_{(-\alpha, \alpha)}^{\beta}\right] \supset\left[X_{(-\alpha, \alpha)}, B_{(-\alpha, \alpha)}^{\beta+1}\right], 0 \leq \beta<\alpha^{\prime}$, (a cofinal system of closed neighborhoods on the right at $\left.X_{(-\alpha, \alpha)}\right)$ and is at the same time the intersection of some $\alpha^{\prime \prime}$-sequence of embedded closed intervals $\left[A_{(-\alpha, \alpha)}^{\beta}, X_{(-\alpha, \alpha)}\right] \supset\left[A_{(-\alpha, \alpha)}^{\beta+1}, X_{(-\alpha, \alpha)}\right], 0 \leq \beta<\alpha^{\prime \prime}, \omega \leq \alpha^{\prime \prime} \leq$ $\alpha$, (a cofinal system of closed neighborhoods on the left at $X_{(-\alpha, \alpha)}$ ) then $B_{(-\alpha, \alpha)}^{\beta}$ and $A_{(-\alpha, \alpha)}^{\beta}$ are such $\alpha^{\prime}$ - and $\alpha^{\prime \prime}$-sequences, respectively. Notice that there are elements $X_{(-\alpha, \alpha)} \in R_{\alpha}$ for which $\alpha^{\prime}=\alpha^{\prime \prime}=\alpha$ (generalized integers, generalized irrationals, some generalized rationals), but there are elements $X_{(-\alpha, \alpha)} \in R_{\alpha}$ for which $\alpha^{\prime}<\alpha$ or $\alpha^{\prime \prime}<\alpha$ though not at the same time; i.e., if $\alpha^{\prime}<\alpha$, then $\alpha^{\prime \prime}=\alpha$ or if $\alpha^{\prime \prime}<\alpha$, then $\alpha^{\prime}=\alpha$ (e.g., $\frac{1}{2^{\omega}}, 1-\frac{1}{2^{\omega}}$, etc.)

Definition 23. A generalized real number $X_{(-\alpha, \alpha)} \in R_{\alpha}$ is a limit of $\alpha^{\prime}$-sequence $S_{\beta}, 0 \leq \beta<\alpha^{\prime} \leq \alpha$, notation $X_{(-\alpha, \alpha)}=\lim _{\beta \rightarrow \alpha^{\prime}} S_{\beta}$, if for each open interval $\left(A_{(-\alpha, \alpha)}, B_{(-\alpha, \alpha)}\right), A_{-(\alpha, \alpha)}<B_{(-\alpha, \alpha)}$, which contains $X_{(-\alpha, \alpha)}$, there exists an ordinal $0 \leq \beta_{0}<\alpha^{\prime}$ such that $S_{\beta} \in\left(A_{(-\alpha, \alpha)}, B_{(-\alpha, \alpha)}\right)$, for all $\beta_{0}<\beta<\alpha^{\prime}$.

In this case a $\alpha^{\prime}$-sequence $S_{\beta}, 0 \leq \beta<\alpha^{\prime} \leq \alpha$, is called convergent and $X_{(-\alpha, \alpha)} \in R_{\alpha}$ is its limit. Clearly, if the $\alpha^{\prime}$-sequence $S_{\beta}, 0 \leq \beta<\alpha^{\prime} \leq \alpha$, converges to $X_{(-\alpha, \alpha)} \in R_{\alpha}$, then this limit is unique.

Using the classical arguments we can easily prove the following theorems.

Theorem 11. A mapping $f: R_{\alpha} \rightarrow R_{\alpha}$ is continuous in the ordering topology if and only if for each element $X_{(-\alpha, \alpha)} \in R_{\alpha}$ and every $\alpha^{\prime}$-sequence $S_{\beta}, 0 \leq \beta<\alpha^{\prime} \leq \alpha$, such that $\lim _{\beta \rightarrow \alpha^{\prime}} S_{\beta}=X_{(-\alpha, \alpha)}$, then $\lim _{\beta \rightarrow \alpha^{\prime}} f\left(S_{\beta}\right)=$ $f\left(X_{(-\alpha, \alpha)}\right)$.

Proof. Let $f: R_{\alpha} \rightarrow R_{\alpha}$ be a continuous mapping and $X_{(-\alpha, \alpha)}$ an arbitrary element in $R_{\alpha}$. Consider any open interval $\left(A_{(-\alpha, \alpha)}^{\prime}, B_{(-\alpha, \alpha)}^{\prime}\right)$ which contains $f\left(X_{(-\alpha, \alpha)}\right)$ and find an open interval $\left(A_{(-\alpha, \alpha)}, B_{(-\alpha, \alpha)}\right)$ which contains $X_{(-\alpha, \alpha)}$ such that $f\left(A_{(-\alpha, \alpha)}, B_{(-\alpha, \alpha)}\right) \subseteq\left(A_{(-\alpha, \alpha)}^{\prime}, B_{(-\alpha, \alpha)}^{\prime}\right)$. Since $\lim _{\beta} S_{\beta}=X_{(-\alpha, \alpha)}$ there exists an ordinal number $\beta_{0}$ such that $S_{\beta} \in$ 
$\left(A_{(-\alpha, \alpha)}, B_{(-\alpha, \alpha)}\right)$, for all $\beta_{0}<\beta<\alpha^{\prime} \leq \alpha$. Then evidently $f\left(S_{\beta}\right) \in$ $\left(A_{(-\alpha, \alpha)}^{\prime}, B_{(-\alpha, \alpha)}^{\prime}\right)$, for all $\beta_{0}<\beta<\alpha^{\prime} \leq \alpha$.

Conversely, suppose the opposite, i.e., for every $\alpha^{\prime}$-sequence $S_{\beta}, 0 \leq \beta<$ $\alpha^{\prime} \leq \alpha$, such that $\lim _{\beta \rightarrow \alpha^{\prime}} S_{\beta}=X_{(-\alpha, \alpha)}$ we have $\lim _{\beta \rightarrow \alpha^{\prime}} f\left(S_{\beta}\right)=f\left(X_{(-\alpha, \alpha)}\right)$; however $f$ is not continuous at some point $X_{(-\alpha, \alpha)}$ in $R_{(-\alpha, \alpha)}$. Evidently, $f$ is not continuous on the right at $X_{(-\alpha, \alpha)}$ or on the left at $X_{(-\alpha, \alpha)}$. We can consider the first case; the second is similar. Thus, if $f$ is not continuous on the right at $X_{(-\alpha, \alpha)}$, then there is an open interval $\left(A_{(-\alpha, \alpha)}^{\prime}, B_{(-\alpha, \alpha)}^{\prime}\right)$ which contains $f\left(X_{(-\alpha, \alpha)}\right)$ such that for every embedded closed interval $\left[X_{(-\alpha, \alpha)}, B_{(-\alpha, \alpha)}^{\beta}\right], 0 \leq \beta<\alpha^{\prime} \leq \alpha$, which is cofinal in the system of all neighborhoods on the right at $X_{(-\alpha, \alpha)}$, we have $f\left(\left[X_{(-\alpha, \alpha)}, B_{(-\alpha, \alpha)}^{\beta}\right]\right)$ is not a subset of $\left(A_{(-\alpha, \alpha)}^{\prime}, B_{(-\alpha, \alpha)}^{\prime}\right)$. Choosing in each $\left[X_{(-\alpha, \alpha)}, B_{(-\alpha, \alpha)}^{\beta}\right]$ an element $S_{\beta}$, such that $f\left(S_{\beta}\right) \notin\left(A_{(-\alpha, \alpha)}^{\prime}, B_{(-\alpha, \alpha)}^{\prime}\right), 0 \leq \beta<\alpha^{\prime} \leq \alpha$, we see that $\lim _{\beta \rightarrow \alpha^{\prime}} S_{\beta}=X_{(-\alpha, \alpha)}$ but $\lim _{\beta \rightarrow \alpha^{\prime}} f\left(S_{\beta}\right) \neq f\left(X_{(-\alpha, \alpha)}\right)$, which is in contradiction with our assumption; i.e., if for an $\alpha^{\prime}$-sequence $S_{\beta}$ we have $\lim _{\beta \rightarrow \alpha^{\prime}} S_{\beta}=X_{(-\alpha, \alpha)}$, then $\lim _{\beta} f\left(S_{\beta}\right)=f\left(X_{(-\alpha, \alpha)}\right)$.

Theorem 12. Let $X_{(-\alpha, \alpha)}$ and $Y_{(-\alpha, \alpha)}$ be elements of $R_{\alpha}$. If

$$
f:\left[X_{(-\alpha, \alpha)}, Y_{(-\alpha, \alpha)}\right] \rightarrow R_{\alpha}
$$

be a continuous mapping such that $f\left(X_{(-\alpha, \alpha)}\right)$ and $f\left(Y_{(-\alpha, \alpha)}\right)$ have different signs, i.e., - and + or + and - , respectively, then there exists an element $Z_{(-\alpha, \alpha)}$ in $R_{\alpha}$ such that $f\left(Z_{(-\alpha, \alpha)}\right)=0_{(-\alpha, \alpha)}$.

Proof. If in the interval $\left[X_{(-\alpha, \alpha)}, Y_{(-\alpha, \alpha)}\right]$ there are no integer ordinal numbers except $X_{(-\alpha, \alpha)}$ and $Y_{(-\alpha, \alpha)}$, then the proof is classical here and is given by the method of our generalized dichotomy, or halving the intervals of the unit one. Comp., [20], Theorem 25, p. 41. If there are such integers, then choose one of them; let it be $Z_{(-\alpha, \alpha)}$ such that $X_{(-\alpha, \alpha)}<$ $Z_{(-\alpha, \alpha)}<Y_{(-\alpha, \alpha)}$. Clearly, $f\left(X_{(-\alpha, \alpha)}\right)$ and $f\left(Z_{(-\alpha, \alpha)}\right)$ or $f\left(Z_{(-\alpha, \alpha)}\right)$ and $f\left(Y_{(-\alpha, \alpha)}\right)$ have different signs, and we choose that interval and denote it by $\left[X_{(-\alpha, \alpha)}^{1}, Y_{(-\alpha, \alpha)}^{1}\right]$. We continue this transfinite process up to the first case where there are no integer ordinal numbers except $X_{(-\alpha, \alpha)}^{\beta}$ and $Y_{(-\alpha, \alpha)}^{\beta}$, $1 \leq \beta<\alpha$; then the proof is also classical and is given by the method of our generalized dichotomy or halving intervals of the unit one. Since we obtain a system of embedded closed intervals whose lengths converge to $0_{(-\alpha, \alpha)}$, by Theorem 8 , there exists a unique element $Z_{(0, \alpha)} \in R_{\alpha}$ such that it belongs to all these intervals. By the continuous property of $f, f\left(Z_{(0, \alpha)}\right)$ 
has at the same time different signs, which is possible only in the case where $f\left(Z_{(0, \alpha)}\right)=0_{(-\alpha, \alpha)}$.

One can consider other classical theorems of Mathematical Analysis in the case of $R_{\alpha}$ since for $\xi=1$ we have the usual real numbers $\mathbb{R}=R_{\omega}$.

Theorem 13. Every closed interval $\left[A_{(-\alpha, \alpha)}, B_{(-\alpha, \alpha)}\right]$ of $R_{\alpha}$ is compact.

Proof. Without loss of generality we can assume that $A_{(-\alpha, \alpha)}$ and $B_{(-\alpha, \alpha)}$ are positive or negative ordinals and $A_{(-\alpha, \alpha)}<B_{(-\alpha, \alpha)}$. Moreover, we can restrict the proof to the case where $\left[A_{(-\alpha, \alpha)}, B_{(-\alpha, \alpha)}\right] \subset R_{\alpha}^{+}$. Assume now that $\gamma$ is a covering of $\left[A_{(-\alpha, \alpha)}, B_{(-\alpha, \alpha)}\right] \subset R_{\alpha}^{+}$consisting of open sets in $R_{\alpha}$ which has no finite subcovering. Then we divide this interval by the point $A_{(-\alpha, \alpha)}+1$, i.e., $\left[A_{(-\alpha, \alpha)}, B_{(-\alpha, \alpha)}\right]=\left[A_{(-\alpha, \alpha)}, A_{(-\alpha, \alpha)}+\right.$ 1] $\cup\left[A_{(-\alpha, \alpha)}+1, B_{(-\alpha, \alpha)}\right]$, the next $\left[A_{(-\alpha, \alpha)}+1, B_{(-\alpha, \alpha)}\right]$ by $A_{(-\alpha, \alpha)}+2$, etc. By Theorem 9 , restrictions of $\gamma$ on $\left[A_{(-\alpha, \alpha)}, A_{(-\alpha, \alpha)}+1\right],\left[A_{(-\alpha, \alpha)}+\right.$ $\left.1, A_{(-\alpha, \alpha)}+2\right], \ldots,\left[A_{(-\alpha, \alpha)}+n, A_{(-\alpha, \alpha)}+n+1\right], \ldots$ have finite subcoverings; we conclude that the restrictions of $\gamma$ on each $\left[A_{(-\alpha, \alpha)}+n, B_{(-\alpha, \alpha)}\right]$ have no finite subcoverings, $0 \leq n<\omega$. Hence $\left[A_{(-\alpha, \alpha)}+\omega, B_{(-\alpha, \alpha)}\right]$ has no finite subcovering of the restriction of $\gamma$, if of course $A_{(-\alpha, \alpha)}+\omega<B_{(-\alpha, \alpha)}$. If $A_{(-\alpha, \alpha)}+\omega=B_{(-\alpha, \alpha)}$, then $B_{(-\alpha, \alpha)}$ is covered by one element of $\gamma$ and consequently, $\left[A_{(-\alpha, \alpha)}+n, B_{(-\alpha, \alpha)}\right]$ covers by this element for some natural $n$. Contradiction. So we continue this transfinite process, passing all possible limit ordinals and $B_{(-\alpha, \alpha)}$ with the same argument. If $B_{(-\alpha, \alpha)}$ is not a limit ordinal, then the impossibility of choosing a finite subcovering from the restriction of $\gamma$ on $\left[B_{(-\alpha, \alpha)}-1, B_{(-\alpha, \alpha)}\right]$ contradicts Theorem 9 .

Theorem 14. A subspace $X$ of $R_{\alpha}$ is a compact Hausdorff space if and only if it is a bounded closed subset of $R_{\alpha}$.

The Proof is classical, except with reference to Theorem 13.

Theorem 15. Every continuous image of a compact Hausdorff space is a compact Hausdorff space.

The Proof is classical.

Theorem 16. Every bounded subset $S$ of $R_{\alpha}$ has a smallest upper bound $M_{(0, \alpha)}=\sup S$ and a greatest lower bound $m_{(0, \alpha)}=\inf S$ in $R_{\alpha}$.

Proof. It is a consequence of Theorem 5 .

Theorem 17. Every continuous function $f$ defined on a compact subset $X$ of $R_{\alpha}$ is bounded and reaches its maximum and minimum values.

Proof. By Theorem 16, the image $S=f(X)$ is a compact Hausdorff space of $R_{\alpha}$ and hence, by Theorem 15, bounded. We apply Theorem 17 and obtain $M_{(-\alpha, \alpha)}=\sup X$ and $m_{(-\alpha, \alpha)}=\inf X$ which by compactness of $S$ belong to $f(S)$. Since $f: X \rightarrow S$ is a surjection $f$, evidently reaches its maximum and minimum values. 
Remark 22. If $\alpha=\omega_{\xi}$, where $\omega_{\xi}$ is the initial ordinal number, $\xi \geq 1$, then our $R_{\omega_{\xi}}$ differs from the space denoted by $R_{\xi}$ in [29] Hausdorff, [1908], because for the latter $\operatorname{dim} R_{\xi}=0$ and for the former $\operatorname{dim} R_{\omega_{\xi}}=1$.

Theorem 18. The class $Q_{\Omega}$ is an $\aleph_{\kappa}$-universal linear ordering for all cardinals $\aleph_{\kappa}, \kappa \in \mathbf{O n}$.

The Proof is a consequence of Mendelson's theorem: $Q_{\kappa}$ is an $\aleph_{\kappa}$-universal linear ordering (see [39] and [53], p. 169).

We omit another way to construct a hierarchy of generalized real numbers of different powers, which are one-dimensional and continuous but different from these presented above, which we had originally planned to show in the present paper. Moreover, algebraic operations would be completely defined, as in Conway's approach [20], but through fundamentally different method. The field strucrure will be on that generalized real numbers and even the completions of fields but all of then except our real numbers $\mathbb{R}$ will be zero-dimensional. There is also a conjecture that Conway's numbers, which come from Game Theory, are subclasses of these new generalized real numbers and all Conway's numbers which is a Field, i.e., with a domain as a class will have a completion which is a FIELD in a more general sense, like a hyperclass. Since this paper is already too long, and a construction of generalized real numbers with complete operetions is beyond the scope of skand theory, we plan to publish the new material in another paper in the near future.

\section{Acknowledgement}

The author wishes to thank Professor Philip T. Grier (USA) for help in editing the manuscript.

\section{REFERENCES}

[1] P. Aczel, Non-Well-Founded Sets. CSLI Lecture Notes Number 14. Stanford: CSLI Publication, 1988.

[2] P. S. Alexandroff, B. A. Pasynkov, Introduction to Dimension Theory. "Nauka", Moscow, 1973 (in Russian).

[3] J. Barwise and J. Etchemendy, The Liar. An Essay on Truth and Circularity. Oxford University Press, New York, Oxford, 1987.

[4] J. Barwise, L. Moss, Vicious Circles. On the Mathematics of NonWellfounded Phenomena. CSLI Lecture Notes Number 60, Stanford, California, 1996.

[5] P. Bernays, A system of axiomatic set theory, J.S.L., II, 6 (1941), 1-17.

[6] U. Blau, The Significance of the Largest and Smallest Numbers for the Oldest Paradoxes, in "One Hundred Years of Russell's Paradox. Mathematics, Logic, Philosophy", Editor Godehard Link. Walter de Gruyeter, Berlin, New York, 2004. 
[7] M. Boffa, Sur la théorie des ensembles sans axiome de fundement, Acta Math., vol. 2 (1969), 415-429.

[8] M. Boffa, Forcing et negation de l'axiome de Fondement, Memoire Acad. Sci. Belg., tome XL, 1972, fasc. 7.

[9] G. Boolos, The iterative conception of set, In Philosophy of mathematics, 1983, Cambridge University Press, London New York New Rochelle Melmourn Sydney, 1983, 486-502.

[10] G. Boolos, Logic, Logic and Logic, Harvard University Press, Cambridge, Massachussetts, London, England, 1998.

[11] N. Bourbaki, Topologie générale ch. IX. Paris, 1948.

[12] N. Bourbaki, Théorie des ensembles. Paris, 1954.

[13] C. Burali-Forti, Una questione sui numeri trasfiniti, Rendic. Palermo 11 (1897) 154-164.

[14] G. Cantor, Über unendliche lineare Punktmannigfaltigkeiten, Math. Ann. 2, Bd. 17 (1880), 355-358.

[15] G. Cantor, Beiträge zur Begründung der transfiniten Mengenlehre, Math. Ann., Bd.46 (1895) 481-517.

[16] G. Cantor, Beiträge zur Begründung der transfiniten Mengenlehre, Math. Ann., Bd.49 (1897) 207-246.

[17] G. Cantor, Gesammelte Abhandlungen mathematischen und philosophischen Inhalts. Ed. by E. Zermelo. Berlin, 1932, 486 pp.

[18] R. B. Chuaqui, Axiomatic Set Theory. Inpredicative Theories of Classes. North-Holland Publishing Company. Amsterdam, New York, Oxford, 1981.

[19] P. J. Cohen, Set Theory and the Continuum Hypothesis. W. A. Benjamin, INC. New York, Amsterdam, 1966.

[20] J. H. Conway, On Numbers and Games. New York: Academic Press, 1976.

[21] C. W. Dodge, Sets, Logic, \& Numbers. Prindle, Weber \& Schmidt, Boston, 1969.

[22] Von Hj. Eklund, Über Mengen, die Elemente ihrer selbst sind, Nyt Tidsskrift f. Mat., Avd., 29 (1918) 8-28.

[23] R. Engelking, General Topology. Warszawa, 1977.

[24] J. Ferreirós, Labyrinth for Thought. A History of Set Theory and Its Role in Modern Mathematics. Birkhäuser, Basel-Boston-Berlin, 2007, 466 pp.

[25] P. Finsler, Über die Grundlagen der Mengenlehre, I. Math. Zeitschrift 25 (1926) 683-713.

[26] M. Forti, F. Honsell, Set Theory with Free Construction Priciples, Annali Scuola Normale Superiore di Pisa, Classe di Scienze, 10 (1983) 493-522.

[27] A. A. Fraenkel, Y. Bar-Hillel, A. Levy, Foundation of set theory. Elsevier, Amsterdam London New York Oxford Paris Shannon Tokyo, 1973.

[28] P. R. Halmos, Naive Set Theory. Van Nostrand Reinhold Company Regional Offices: Cincinnati, New York, Chicago, Melbourne, Dalas, Litton Educational Publishing, INC., 1960.

[29] F. Hausdorff, Grundzüge eine Theorie der geordneten Mengen, Ann. Math. 65 (1908), 435-505.

[30] F. Hausdorff, Grundzüge der Mengenlehre. Leipzig, 1914.

[31] H. Herrlich, Ordunungsfähigkeit total-diskontinuierlich Räume, Math. Ann. 159 (1965), 77-80. 
[32] G. Hessenberg, Grudbegriffe der Mengenlehre, Abh. der Friesschen Schule, N.S. [1], Heft 4, Göttingen, 1906, 220 pp.

[33] D. Hilbert, Logische Principien des mathematischen Denkens, Lecture course in the summer semester 1905, lecture notes by Ernst Hellinger. Library of the Mathematics Seminar of the University of Göttingen, 1905.

[34] Ph. E. B. Jourdain, Contributions to the founding of the set theory of thransfinite numbers by Georg Cantor. Dover Publication, Inc., New York, 1915.

[35] M. Katětov, On dimension of metric spaces, DAN SSSR, 78, \# 2 (1951), 189-191 (in Russian).

[36] K. Kuratowski, A. Mostowski, Set Theory. North-Holland Publishing Company, Amsterdam, 1967.

[37] G. Landini, Russell's Hidden Substitutional Theory. Oxford University Press, New York Oxford 1998.

[38] Ju. T. Lisica, The paradox of Russell's paradox. Skands and classes of skands as extensions of Mirimanoff's extraordinary sets, 12 pp. (unpublished paper).

[39] E. Mendelson, On a class of universal ordered sets, Proc. Amer. Math. Soc., 9 (1958), 712-713.

[40] E. Mendelson, The axiom of Fundierung and the axiom of choice, Arch. Math. Logik Grundlagenforsch, 4, (1958), 65-70.

[41] E. Mendelson, Introduction to Mathematical Logic. Chapman and Hall, London, Weinheim, New York, Tokyo, Melbourne, Madras 1997.

[42] R. Milner, A Calculus of Communicating Systems. Berlin: Springer-Verlag, Lecture Notes in Computer Science, 92, 1980.

[43] D. Mirimanoff, Les antinomies de Russell et de Burali-Forti et le problème fondumentale de la théorie des ensembles, L'Enseignement Mathématique, 19 (1917) 37-52.

[44] D. Mirimanoff, Remarques sur la théorie des ensembles et les antinomies cantoriennes. I, L'Enseignement Mathématique, 19 (1917) 209-217.

[45] D. Mirimanoff, Remarques sur la théorie des ensembles et les antinomies cantoriennes. II, L'Enseignement Mathématique, 21 (1920) 29-52.

[46] A. P. Morse, A theory of sets. New York, 1965, 130 pp.

[47] A. Mostowski, Über die Anabhängigkiet des Wohlordnungssatzes vom Ordnungsprinzip, Fund. Math. 32 (1939) 201-252.

[48] J. Mycielski, Russell's Paradox and Hilbert's (much Forgotten) View of Set Theory, in "One Hundred Years of Russell's Paradox. Mathematics, Logic, Philosophy", Editor Godehard Link. Walter de Gruyeter, Berlin, New York, 2004.

[49] J. von Neuman, Zur Einführung der transfiniten Zahlen, Acta Litt. Ac. Sci. Hung. Fran. Joseph., 1 (1923) 199-208.

[50] J. von Neuman, Eine Axiomatisierung der Mengelehre, J. reine angew. Math., 154 (1925) 219-240.

[51] W. Neumer, Über den Aufbau der Ordnungszhalen, Math. Zeitschr. 53 (1951) 59-69.

[52] A. Poincaré, Science et Méthode. Paris, 1908, 311 pp. 
[53] J. G. Rosenstein, Linear Orderings. Academic Press, New York-LondonParis-San Diego-San Fracisco-San Paulo-Sydney-Tokio-Toronto, 1982, 487 pp.

[54] B. Russell, The Principles of Mathematics. New York W. W. Norton \& Company, INC Publishers, 1903.

[55] D. Scott, A Different Kind of Model for Set Theory, Unpublished paper given at the 1960 Stanford Congress of Logic, Methodology and Philosophy of Science.

[56] W. Sierpiński, Cardinal and ordinal numbers. Warszawa, 1965.

[57] N. Vedenissoff, Remarks on the dimension of topological spaces, Uch. Zapiski Mosk. Univ. 30 (1939), 131-140 (in Russian).

[58] A. N. Whitehead, B. Russell, Principia Mathematica, Volume I. Cambridge of the University Press, Cambridge, 1910.

[59] E. Zermelo, Übersuchungen über Grundlagen den Mengenlehre, I, Math. Ann., 65 (1908), 261-281.

[60] E. Zermelo, Über Grenzzahlen und Menchenbereiche, Fund. Math., 16 (1930), 29-47.

[61] "One Hundred Years of Russell's Paradox. Mathematics, Logic, Philosophy", Editor Godehard Link. Berlin, New York, 2004.

[62] Briefwechsel Cantor - Dedekind/Hrsg. von E. Noether, J. Cavaillès. Paris: Hermann, 1937.

[63] Zermelo to Scholz, dated Freiburg i. Br., 10 April 1936. Institut für mathematische Logik und Grundlagenforschung, Münster, Scholz papers.

[64] Paradoxes and Contemporary Logic. Stanford Encyclopedia of Philosophy, Metaphysics Research Lab, CSLI, Stanford University, 2007. 


\title{
СКАНД ТЕОРИЈА И НЕЈЗИНИ ПРИМЕНИ. (НОВ ПОГЛЕД МНОЖЕСТВАТА ШТО НЕ СЕ ДОБРО ОСНОВАНИ)
}

\author{
J. Т. Лисица
}

Р е $з$ и м е

Воведен е нов математички објект наречен сканд, кој во општ случај е множество што не е добро основано. Скандовите со конечна должина се се стандардни добро основани множества а скандовите со многу голема должина (како хипер-скандот од сите ординали) се хипер-класи.

Разгледани си и себе-сличните скандови и тие ја разјаснуваат рефлексивноста на множества, т.е. значењето на релацијата $X \in X$; посебно себе-слицните скандови разгледани како множества што не се добро основани се секогаш рефлексивни, но не важи обратно. Постоењето на себеслични скандови докажува дека добро познатите парадокси од теоријата на множества воопшто не се парадокси и според тоа не мора да се фатални за секоја теорија на множества. Значи докажана е неконзистентностга на "множеството" на Расел $R=\{X \mid X \notin X\}$ не со помош на Раселовиот парадокс (како што е традиционално даден, и е погрешен), но со едноставен метод на максималност (универзалност) на $R$ што враќа на Канторовиот и е применет и на другите парадокси од теоријата на множества.

Дефинирани се и воопштените скандови и е демонстриран нов поглед на воопштената сканд-класа од сите ординали. Специјално, дефиниран е последниот ординал наречен eschaton.

Следната примена на теорината на скандови е во описот на сите епсилон-броеви во смисла на Кантор. Друга примена е обопштената теорија на еднодимензионален континуум од произволен степен и конструкција на обопштени реални броеви како неархимедова права од произволен степен, и воведувањето на апсолутен континуум и на апсолутна права како хипер-класа најблиска до класата од множества.

Mathematical Analysis and Function Theory Department, Russian UniverSity of Peoples' Friendship, Miklukho-Maklay str. 6, 117198 Moscow, Russia,

E-mail address: jutlisica@yandex.ru 